\title{
A model of quantum gravity with emergent spacetime
}

\author{
Sung-Sik Lee \\ Department of Physics \& Astronomy, McMaster University, \\ Hamilton ON, Canada \\ Perimeter Institute for Theoretical Physics, \\ Waterloo ON, Canada \\ E-mail: slee@mcmaster.ca
}

AbStRaCT: We construct a model of quantum gravity in which dimension, topology and geometry of spacetime are dynamical. The microscopic degree of freedom is a real rectangular matrix whose rows label internal flavours, and columns label spatial sites. In the limit that the size of the matrix is large, the sites can collectively form a spatial manifold. The manifold is determined from the pattern of entanglement present across local Hilbert spaces associated with column vectors of the matrix. With no structure of manifold fixed in the background, the spacetime gauge symmetry is generalized to a group that includes diffeomorphism in arbitrary dimensions. The momentum and Hamiltonian that generate the generalized diffeomorphism obey a first-class constraint algebra at the quantum level. In the classical limit, the constraint algebra of the general relativity is reproduced as a special case. The first-class nature of the algebra allows one to express the projection of a quantum state of the matrix to a gauge invariant state as a path integration of dynamical variables that describe collective fluctuations of the matrix. The collective variables describe dynamics of emergent spacetime, where multi-fingered times arise as Lagrangian multipliers that enforce the gauge constraints. If the quantum state has a local structure of entanglement, a smooth spacetime with well-defined dimension, topology, signature and geometry emerges at the saddle-point, and the spin two mode that determines the geometry can be identified. We find a saddle-point solution that describes a series of $(3+1)$-dimensional de Sitter-like spacetimes with the Lorentzian signature bridged by Euclidean spaces in between. The phase transitions between spacetimes with different signatures are caused by Lifshitz transitions in which the pattern of entanglement is rearranged across the system. Fluctuations of the collective variables are described by bi-local fields that propagate in the spacetime set up by the saddle-point solution.

Keywords: AdS-CFT Correspondence, Models of Quantum Gravity, Space-Time Symmetries

ArXiv EPrint: 1912.12291 


\section{Contents}

1 Introduction 1

1.1 Conceptual overview 3

$\begin{array}{lll}1.2 & \text { Outline } & 7\end{array}$

2 Kinematics $\quad 9$

2.1 Hilbert space 9

2.2 Frame 9

2.3 Local structure 11

3 Gauge symmetry 13

3.1 Review of the Hamiltonian formalism of the general relativity 13

$\begin{array}{ll}3.2 \text { Momentum constraint } & 15\end{array}$

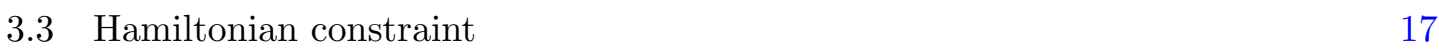

$\begin{array}{lll}3.4 & \text { First-class constraint algebra } 20\end{array}$

4 Path integral representation of state projection $\quad 22$

4.1 Projection 22

4.2 Gauge invariant local structure 23

4.3 Path integration of collective variables 24

4.4 Gauge invariance of the action 26

5 Spacetime diffeomorphism and emergent geometry 28

5.1 Momentum density 28

$\begin{array}{lll}5.2 & \text { Hamiltonian density } & 29\end{array}$

$\begin{array}{lll}5.3 & \text { Emergent metric } & 30\end{array}$

6 Classical equation of motion $\quad 31$

6.1 Symmetry of semi-classical states 31

6.2 Saddle-point equation of motion 33

$\begin{array}{ll}6.3 \text { Gauge fixing } & 35\end{array}$

7 Translationally invariant solution $\quad 36$

$\begin{array}{lll}7.1 & \text { Numerical solution } & 39\end{array}$

$\begin{array}{lll}7.2 & \text { Analytic solution } & 42\end{array}$

7.3 Emergent Lorentz symmetry 44

$\begin{array}{lll}7.4 & \text { Effective theory } & 45\end{array}$

8 Summary and discussion $\quad 49$

$\begin{array}{lll}8.1 \mathrm{dS} / \mathrm{CFT} & 49\end{array}$

$\begin{array}{lll}8.2 & \mathrm{AdS} / \mathrm{CFT} & 50\end{array}$

$\begin{array}{lll}8.3 & \text { Open questions } & 51\end{array}$ 
A Derivation of eq. (3.21) $\quad \mathbf{5 2}$

B Non-normalizability of gauge invariant state 52

C Computation of the commutator of the Hamiltonians 53

D Poisson brackets of the momentum and Hamiltonian $\quad 55$

E Derivations of the transformed shift and lapse functions $\quad 56$

E.1 Eq. (5.6) 56

$\begin{array}{lll}\text { E.2 Eq. (5.7) } & 56\end{array}$

E.3 Eq. (5.15) 57

$\begin{array}{lll}\text { E.4 Eq. (5.16) } & 57\end{array}$

$\begin{array}{lll}\text { F } & \text { Metric in translationally invariant states } & 58\end{array}$

G Transformation of the collective variables under boost $\quad 58$

$\begin{array}{lll}\text { G.1 The shift vector } & 58\end{array}$

$\begin{array}{lll}\text { G.2 The lapse function } & 59\end{array}$

$\begin{array}{lll}\text { G.3 } & p_{c} & 59\end{array}$

$\begin{array}{lll}\text { G.4 } & q & 60\end{array}$

\section{Introduction}

According to Einstein's theory of general relativity, gravity originates from dynamical geometry [1]. While the theory has been extremely successful in explaining a myriad of phenomena in the classical regime, understanding the quantum nature of gravity remains an outstanding problem [2-17]. One notoriously difficult problem in quantum gravity is to tame quantum fluctuations at short distance scales while preserving the essential aspects of the general relativity at long distances $[18,19]$. The crucial feature that a successful quantum theory of gravity should reproduce in the continuum limit is the diffeomorphism invariance, which largely fixes the theory at long distance scales.

Quantizing fluctuations of geometry in a fixed dimension, either in the form of metric or a new degree of freedom, has provided important insights into quantum gravity. However, this may not give the complete picture. If metric is dynamical, it is natural to posit that dimension and topology of spacetime are also dynamical. In the presence of strong quantum fluctuations of geometry, there is in priori no reason why topology and dimension of spacetime remain well-defined. Ideally, a full theory of quantum gravity should be able to describe phenomena in which not only geometry but also dimension and topology evolve dynamically. A background independent theory in the strongest sense should include all of dimension, topology and geometry as dynamical degrees of freedom: the entirety of spacetime should emerge [20]. 
Background independent theories can not be local theories [21] because a fixed notion of locality can not be defined without specifying dimension, topology and geometry in the background [22]. Nonetheless, the success of local quantum field theories as a low-energy description of our universe implies that local effective theories should arise as approximate descriptions within states that describe classical spacetimes [23, 24]. The degree of locality in the effective theory should be determined with respect to the metric of the classical geometry. Because the classical geometry is state dependent, so is the locality of the effective field theory. Background independent theories from which local effective theories emerge, while being non-local in the strict sense, should have a weaker notion of locality called relative locality [25]. This may be an essential ingredient that needs to be taken into account in understanding quantum gravity.

The AdS/CFT correspondence [8-10] provides a route to quantum gravity by mapping a quantum field theory without gravity to a string theory that includes dynamical gravity in one higher dimensional space. Although a background independent non-perturbative formulation of the string theory is not known yet, the AdS/CFT correspondence already provides an important clue on the microscopic origin of gravity : geometry is nothing but a coarse-grained variable that controls entanglement (among other things) of ordinary quantum matter [26-32]. This suggests an approach to quantum gravity that we adopt in this paper. Here, the fundamental degrees of freedom are ordinary quantum matter defined on a set. The set is what is to become a spatial manifold, but it does not have a fixed structure of manifold. Dimension, topology and geometry of the set are all collective degrees of freedom of the matter defined on the set. In particular, distances between points in the set are determined from the amount of entanglement between points. Two points that are strongly (weakly) entangled are deemed to be close (far) [25, 33]. The pattern of entanglement determines the connectivity among points in the set. The connectivity, in turn, determines a manifold, if its pattern exhibits a local structure.

Within this framework, a Hamiltonian that governs the dynamics of the underlying quantum matter naturally induces dynamics of collective variables that describe dimension, topology and geometry of the manifold. As much as the underlying quantum matter is dynamical, the emergent manifold is fully dynamical. One main goal in this program is to construct a Hamiltonian for the matter such that the induced Hamiltonian for the collective variables reduces to that of the general relativity in a classical limit. Such a Hamiltonian must be relatively local if it is to induce background independent dynamics for the collective variables and admit a local effective theory description for perturbative fluctuations around semi-classical states [25]. Since geometric distance is nothing but a collective property of the underlying matter, the effective strength of interactions between points should be state dependent. In a state that describes a geometry that gives rise to a small (large) proper distance between two points, the interaction between them should be strong (weak) in the relatively local Hamiltonian.

Recently, a simple relatively local model has been constructed. In the background independent model, the collective variables that describe dynamics of dimension, topology and geometry are classical in the large $N$ limit, where $N$ is the number of flavours of underlying quantum matter [34]. In different states, the same Hamiltonian acts as local 


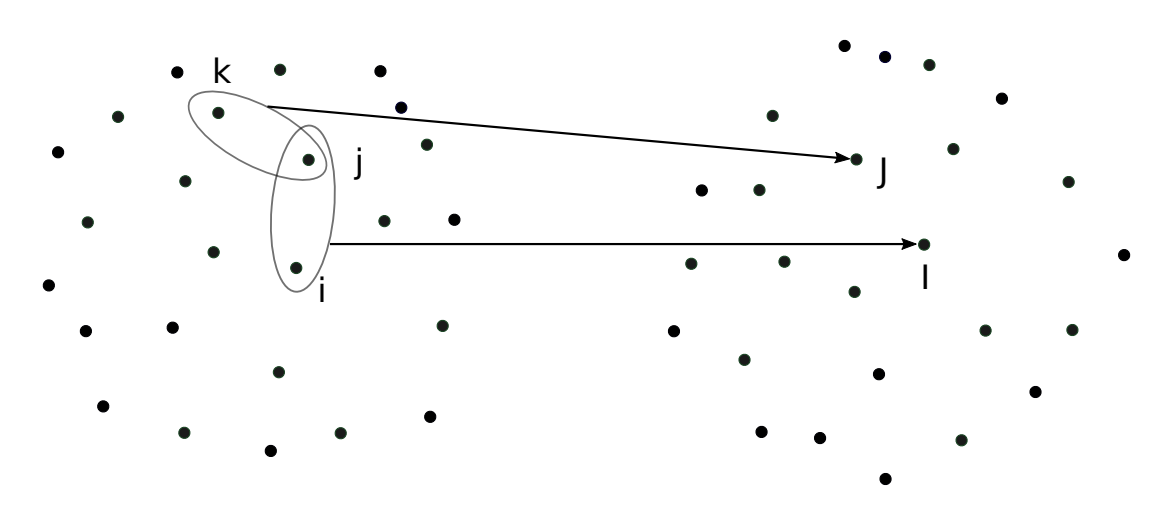

Figure 1. Each column of the $M \times L$ matrix represents a point in the set of $L$ sites on the left panel. At each site, one can define a local Hilbert space spanned by a column of $M$ scalars. Under $\mathrm{SL}(L, \mathbb{R})$ that acts on the matrix from the right, column vectors are linearly superposed to form a new set of $L$ column vectors. The rotated column vectors define a new set of sites and associated local Hilbert spaces shown in the right panel. A choice of local Hilbert spaces into which the total Hilbert space is decomposed is called a frame.

Hamiltonians defined on manifolds with different dimensions, topologies and geometries. However, the model is not a theory of gravity yet because it lacks the diffeomorphism invariance. The goal of this paper is to construct a relatively local model of quantum gravity. For other related approaches to quantum gravity, see refs. [33, 35-38].

\subsection{Conceptual overview}

In this section, we provide a conceptual overview without technical details.

The microscopic degree of freedom of the theory is a real rectangular matrix with $M$ rows and $L$ columns with $M \gg L \gg 1$. While the row index labels internal flavours, the column index plays the role of sites. Each column defines a local Hilbert space spanned by states of $M$ real scalars. A choice of such local Hilbert spaces is called a frame. There are multiple ways to choose a frame as $L$ column vectors can be linearly superposed to form a different set of column vectors. A set of rotated vectors form a new frame. Rotations of frame that preserve the norm of the Hilbert space are generated by the special linear group, $\operatorname{SL}(L, \mathbb{R})$ that multiplies the matrix from the right. Once a frame is chosen, the total Hilbert space can be written as a direct product of the local Hilbert spaces defined in that frame. ${ }^{1}$ This is illustrated in figure 1.

In a frame, the collection of sites can form a spatial manifold for $L \gg 1$. However, no structure of manifold, not even its existence, is fixed in the theory. Instead, the existence of spatial manifold and its structure, if exists, depend on quantum state of the matrix in the following way. Suppose we compute the mutual informations between all pairs of sites in a frame. The mutual information between sites $i$ and $j$ is given by $I_{i j}=S_{i}+S_{j}-S_{i \cup j}$, where $S_{A}$ is the von Neumann entanglement entropy of subset $A$. Then we ask if there exists a Riemannian manifold into which the sites can be embedded such that $-\ln I_{i j}$ is proportional to the proper distance between the images of $i$ and $j$ in the Riemannian manifold to the

\footnotetext{
${ }^{1}$ A frame naturally defines a set of local observers who can access the local Hilbert space at each site.
} 


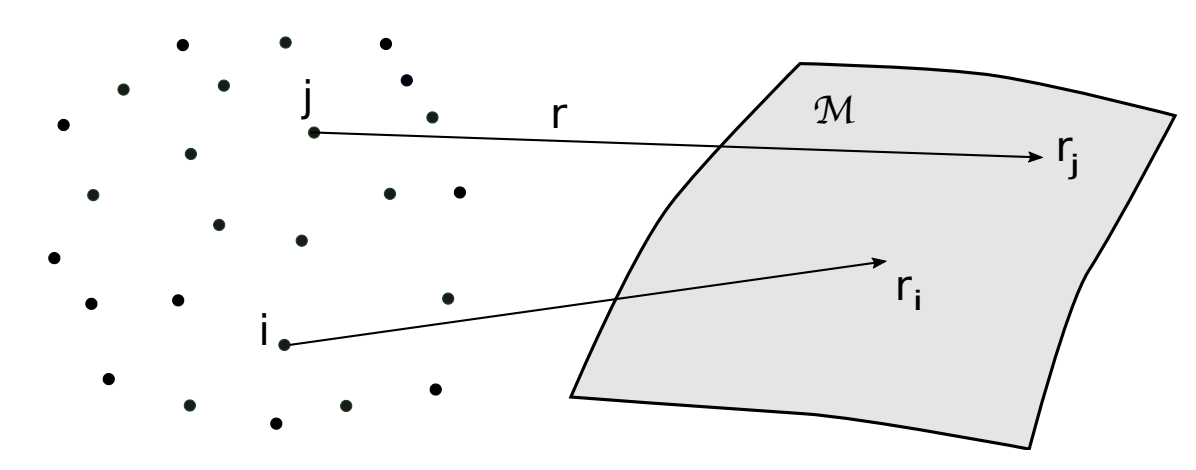

Figure 2. A state has a local structure in a frame if there exists a mapping $r$ from the set of sites into a Riemannian manifold $\mathscr{M}$ such that the mutual information between sites $i$ and $j$ is proportional to $e^{-\frac{d\left(r_{i}, r_{j}\right)}{\xi}}$ to the leading order in $d\left(r_{i}, r_{j}\right)$ for any $i$ and $j$, where $d\left(r_{i}, r_{j}\right)$ is the proper distance between $r_{i}$ and $r_{j}$ in the Riemannian manifold and $\xi$ is a constant.

leading order in the proper distance. ${ }^{2}$ If there exists such a Riemannian manifold, we say that the state has a local structure (see figure 2). Roughly speaking, a state with a local structure is a short-range entangled state when viewed as a state defined on a Riemannian manifold in which sites are embedded. A classical notion of manifold exists only for states with local structures. Any spatial manifold can emerge in this manner. Some examples are given in figure 4. Here, dimension, topology and proper volume of space are order parameters that encode different patterns of entanglement.

In order to identify physical Hilbert space, we need to construct constraints that generate gauge symmetry. We start with the gauge group that generalizes the spatial diffeomorphism. The immediate issue that we face is that the theory does not assume a manifold with fixed dimension and topology. Because a spatial manifold with any dimension can dynamically arise, the gauge group must be general enough to include spatial diffeomorphism in arbitrary dimensions. With this in mind, we consider $\mathrm{O}(M) \times \mathrm{SL}(L, \mathbb{R})$ symmetry, where the orthogonal group acts on the rows of the matrix, and the special linear group on the columns. The $\mathrm{O}(M)$ acts on the internal flavour, and is regarded as a global symmetry of the theory. On the other hand, $\operatorname{SL}(L, \mathbb{R})$ acts on the site index, and is a space symmetry. As $\mathrm{SL}(L, \mathbb{R})$ can rotate a frame into any other frame, it can generate diffeomorphism in any manifold. $\mathrm{SL}(L, \mathbb{R})$ generators that give rise to smooth diffeomorphisms in the continuum limit can be identified in any dimension as is discussed in section 3.2. Therefore, we take $\mathrm{SL}(L, \mathbb{R})$ as the generalized spatial diffeomorphism group.

An unavoidable consequence of the enlarged gauge symmetry is that $\operatorname{SL}(L, \mathbb{R})$ also includes transformations that are non-local in a given coordinate system chosen by the local structure of a state. Under an $\mathrm{SL}(L, \mathbb{R})$ transformation that mixes two columns through linear superpositions, the set of local sites is not preserved. A local Hilbert space in one frame is made of linear superpositions of states defined in multiple local Hilbert

\footnotetext{
${ }^{2}$ The mutual information has been used to extract the bulk geometry in a holographic mapping in ref. [82].
} 
spaces in another frame. Since the total Hilbert space can be written as direct products of local Hilbert spaces in any frame, there is no preferred frame. Consequently, whether a state has a local structure or not is not gauge invariant. Even if a state has a local structure of entanglement in one frame, it does not have a local structure in a rotated frame in general. In order to define a gauge invariant notion of local structure, we first need to choose a frame in a gauge invariant manner. Although there is no preferred frame fixed in the background, one can define a set of local Hilbert spaces in terms of physical degrees of freedom within the theory. This can be done in states in which the $\mathrm{O}(M)$ flavour symmetry is spontaneously broken to a smaller group by a non-zero expectation value in an $L \times L$ block of the $M \times L$ rectangular matrix. If the square matrix formed by the $L \times L$ block is non-singular, a frame can be defined in terms of the row vectors in the block. Since it provides a physical reference with respect to which the notion of local Hilbert spaces are defined, the local structure defined in this frame is gauge invariant.

To fully specify the physical Hilbert space, one also needs to define a Hamiltonian constraint. In the general relativity, the Hamiltonian density forms the representation of a scalar density under the spatial diffeomorphism group. In the present theory, the Hamiltonian should form a representation under the generalized spatial diffeomorphism group, $\mathrm{SL}(L, \mathbb{R})$. The minimal representation that an $\mathrm{O}(M)$ invariant Hamiltonian forms under $\operatorname{SL}(L, \mathbb{R})$ is a rank 2 symmetric tensorial representation. Accordingly, the lapse function is generalized to a rank 2 symmetric tensor, which is called lapse tensor.

In the general relativity, the lapse function controls the lapse of proper time at each location in space, and there are one scalar function worth of ways to generate multi-fingered time evolutions. In the present theory, there are more ways of generating time evolutions because of the off-diagonal elements of the lapse tensor. A general lapse tensor can be rotated into a diagonal form using an $\operatorname{SL}(L, \mathbb{R})$ transformation. The off-diagonal elements of the lapse tensor encode the information on the frame in which lapse tensor is put into a diagonal form. Therefore, the lapse tensor determines not only the speed of local time evolutions but also the frame in which the time evolution is generated. In priori, one can describe time evolution in any frame, and there is no preferred lapse tensor. A gauge invariant notion of time evolution can only be defined in terms of physical clocks made of dynamical degrees of freedom within the theory. If a set of unentangled clocks are prepared out of local degrees of freedom in a frame, the Hamiltonian with a lapse tensor diagonal in that frame should evolve those clocks independently. When the whole system that includes the clocks and other degrees of freedom is evolved with the Hamiltonian, the evolution of the other degrees of freedom relative to the local clocks describes the physical time evolution. The correlation between the evolution of the local clocks and the remaining degrees of freedom is the gauge invariant content of the theory. Different choices of local clocks give rise to different relative motions.

In order to make sure that the Hamiltonian is background independent, and evolves unentangled set of clocks independently, the Hamiltonian must be relatively local in the frames in which the lapse tensor is diagonal. For the background independence, any two sites can couple with each other as there is no fixed notion of locality. However, the effective strength of coupling between two sites should be determined from the entanglement present 
between the sites because the proper distance between the points is determined from the entanglement. If two points are weakly (strongly) entangled, the coupling between them are weak (strong). Although this kind of state dependent evolution generally requires a non-linear action on states, a linear operator can realize the state dependent locality approximately in the limit that the size of the matrix is large. This guarantees that two sites that are unentangled remain decoupled to the leading order in the size of the matrix.

The momentum and Hamiltonian obey a first-class constraint operator algebra. The first-class nature of the constraint algebra allows one to define the set of gauge invariant states without introducing additional constraints. However, gauge invariant states are nonnormalizable with respect to the norm defined for the microscopic degree of freedom. All gauge invariant states have infinite norm because the gauge group, which is non-compact, necessarily generates gauge orbits that are unbounded in the phase space. Any state that has a finite norm must break the gauge symmetry spontaneously. In this case, a natural object to consider is a projection between a state with a finite norm with a gauge invariant state. This can be viewed as a wavefunction of the gauge invariant state written in basis states with finite norms. Alternatively, the projection of the state without gauge invariance toward the gauge invariant state can be implemented by applying momentum and Hamiltonian constraints. The fact that the state with a finite norm is not gauge invariant gives rise to a non-trivial evolution as the state is projected toward the gauge invariant state, where the state with a finite norm plays the role of an initial state defined on a Cauchy surface. This process can be understood as a time evolution, where multi-fingered times arise as Lagrangian multipliers that enforce the gauge constraints.

In order to describe the process of gauge projection, it is useful to consider a subHilbert space that a given 'initial state' explores through the evolution generated by gauge transformations. A sub-Hilbert space that is closed under the operations of the gauge transformations is specified by global symmetry. Here we consider the sub-Hilbert space in which the $\mathrm{O}(M)$ flavour symmetry is broken to $S_{L} \times \mathrm{O}(N / 2) \times \mathrm{O}(N / 2)$, where $S_{L}$ is the permutation group of the first $L$ flavours and $N=M-N$. This specific pattern of broken symmetry is not crucial. Here, this group is chosen as an example that gives rise to a minimum number of propagating degrees of freedom after gauge degrees of freedom is removed. One may choose a smaller unbroken symmetry, in which case there exist more physical degrees of freedom. The sub-Hilbert space with $S_{L} \times \mathrm{O}(N / 2) \times \mathrm{O}(N / 2)$ is the kinematic Hilbert space. It is spanned by basis states which are labeled by a set of collective variables. The collective variables, which are singlets of the unbroken symmetry, control the pattern of entanglement within the sub-Hilbert space. Accordingly, those collective variables encode the information on dimension, topology and geometry of the emergent manifold if the pattern of entanglement has a local structure. Since general states in the sub-Hilbert space can be faithfully represented as linear superpositions of the basis states labeled by the collective variables, the dynamics within the sub-Hilbert space is completely captured by the collective variables. In particular, the momentum and Hamiltonian induce constraints that act on wavefunctions defined in the space of the collective variables. The induced Hamiltonian for the collective variables is background independent as the underlying Hamiltonian for the microscopic degree of freedom is relatively local. 
The projection of a state with a finite norm in the kinematic Hilbert space to a gauge invariant state can be expressed as a path integration over the collective variables that describe fluctuations of spacetime. In the path integration for the collective variables, spacetimes with different dimensions, topologies and geometries are summed over nonperturbatively. In the limit that $M \gg L \gg 1$, the path integration can be replaced with a saddle-point. If the state with a finite norm has a $D$-dimensional local structure, a $(D+1)$-dimensional spacetime manifold emerges at the saddle-point. The collective variables, which are bi-local in space, can be viewed as an infinite tower of local fields that includes a spin 2 mode. In the classical limit, the constraint algebra for the collective variables reduces to the hypersurface deformation algebra of the general relativity to the leading order in the gradient expansion if all other modes except for the spin 2 mode is turned off. From the constraint algebra, one can identify the emergent metric degree of freedom as a composite of the dynamical collective variables. The metric identified from the algebra confirms the idea that the proper distance between sites is determined from the entanglement such that strongly entangled sites are physically close to each other. The saddle-point configuration provides a classical spacetime on which fluctuations of collective variables propagate. The propagating modes are bi-local fields that are described by an effective theory whose locality is determined with respect to the classical geometry set by the saddle-point configuration.

The collective variables are kinematically non-local. They can be viewed as an infinite tower of local fields that include the gravitational degree of freedom and many other degrees of freedom. The gravitational degree of freedom is a collective mode of the matrix that encodes the inter-site entanglement in the spin 2 channel. Other degrees of freedom describe collective modes with different spins. The emergent geometry captures only partial information of the full entanglement pattern. The higher-spin collective modes describe entanglement of the underlying matrix which is not captured by the geometry.

\subsection{Outline}

Here is an outline of the rest of the paper. In section 2, the kinematics of the theory is discussed. We define the full Hilbert space from which the kinematic Hilbert space and the physical Hilbert space are to be defined. We also define the inner product, and introduce the notion of frame. In section 3, we first review the Hamiltonian formulation of the general relativity as we will use the Hamiltonian formalism in this paper. We then construct the generalized momentum and Hamiltonian constraints. From an explicit computation of the commutators between the constraints, it is shown that the constraints obey a first-class operator algebra. In section 4 , we define the kinematic Hilbert space as the subset of states with unbroken $S_{L} \times \mathrm{O}(N / 2) \times \mathrm{O}(N / 2)$ flavour symmetry. It is shown that states that are gauge invariant have infinite norm, and states with finite norms necessarily break the gauge symmetry. The projection of a state with a finite norm in the kinematic Hilbert space to a gauge invariant state is expressed as a path integration over collective variables that describe dynamical spacetime. Multi-fingered time evolutions arise as a sum over possible routes via a state in the kinematic Hilbert space is projected to a gauge invariant state. In section 5 , it is shown that the constraint algebra of the present theory reduces to that of the general relativity in a special case. Based on the algebra that 


\begin{tabular}{|c|c|}
\hline $\begin{array}{c}\text { Fundamental } \\
\text { degree of freedom (d.o.f.) }\end{array}$ & $\begin{array}{c}\text { A real rectangular matrix: }\left\{\Phi^{A}{ }_{i} \mid 1 \leq A \leq M, 1 \leq i \leq L\right\} \\
A \text { : flavour index, } i \text { : site index }(M \gg L \gg 1)\end{array}$ \\
\hline Frame & $\begin{array}{l}\text { A decomposition of the full Hilbert space } \\
\text { into local Hilbert spaces }\end{array}$ \\
\hline Frame rotation & $\mathrm{SL}(L, \mathbb{R})[$ right multiplication on $\Phi]$ \\
\hline Local structure & $\begin{array}{l}\text { A pattern of entanglement that exhibits locality } \\
\text { across local Hilbert spaces [section 2.3] }\end{array}$ \\
\hline Flavour symmetry & $\mathrm{O}(M)$ [left multiplication on $\Phi]$ \\
\hline Kinematic Hilbert space $(\mathscr{V})$ & Space of states with unbroken $S_{L} \times \mathrm{O}\left(\frac{M-L}{2}\right) \times \mathrm{O}\left(\frac{M-L}{2}\right) \subset \mathrm{O}(M)$ \\
\hline Basis states of $\mathscr{V}$ & $\begin{array}{c}\left|s, t_{1}, t_{2}\right\rangle[\text { eqs. }(4.3),(4.5),(4.4)] \\
s, t_{1}, t_{2}: \text { collective variables }(L \times L \text { matrices })\end{array}$ \\
\hline $\begin{array}{l}\text { Generators of spacetime } \\
\text { gauge symmetry }\end{array}$ & $\begin{array}{l}\text { Generalized momentum: } \mathrm{SL}(L, \mathbb{R}) \text { transformation [eqs. }(3.8),(3.10)] \\
\text { Generalized Hamiltonian: frame dependent local time translation [eq. }(3.20) \text { ] }\end{array}$ \\
\hline Constraint algebra (C.A.) & First-class operator algebra [eqs. (3.24), (3.25), (3.27)] \\
\hline C. A. in the classical limit & First-class Poisson algebra [eqs. (4.17), (4.18), (4.19)] \\
\hline $\begin{array}{c}\text { Constraints } \\
\text { in the continuum limit }\end{array}$ & $\begin{array}{l}\text { Momentum constraint [eq. (5.2)] with shift [eq. (3.15)] } \\
\text { Hamiltonian constraint [eq. (5.9)] with lapse [eq. (5.11)] }\end{array}$ \\
\hline $\begin{array}{l}\text { Constraint algebra } \\
\text { in the continuum limit }\end{array}$ & $\begin{array}{l}\text { Generalized hypersurface deformation algebra } \\
{[\text { eqs. }(5.5),(5.14),(5.16)]}\end{array}$ \\
\hline $\begin{array}{l}\text { Projection of a state in } \mathscr{V} \\
\text { to a gauge invariant state }\end{array}$ & $\begin{array}{l}\text { Path integration of the collective variables [eq. (4.14)] } \\
\text { that represents fluctuating spacetime }\end{array}$ \\
\hline Emergent metric & A composite of the collective variables [eqs. (5.18), (5.19)] \\
\hline Saddle-point equation & eq. (6.7) \\
\hline $\begin{array}{l}\text { A classical solution } \\
\text { for a generic initial condition }\end{array}$ & $\begin{array}{l}\text { A series of de Sitter-like spacetimes } \\
\text { bridged by Euclidean spaces [figure 11] }\end{array}$ \\
\hline $\begin{array}{l}\text { A fine-tuned } \\
\text { classical solution }\end{array}$ & $\begin{array}{l}\text { Minkowski spacetimes } \\
\text { [eq. (7.36)] }\end{array}$ \\
\hline Effective theory & Bi-local field theory [eq. (7.45)] \\
\hline
\end{tabular}

Table 1. A roadmap of the paper.

the momentum and Hamiltonian constraints obey in the continuum limit, the emergent metric degree of freedom is identified in terms of the collective variables. In the limit that the size of the matrix is large, the dynamical collective variables become classical. In section 6 , the saddle-point equation of motion for the collective variables is derived. The equation of motion is solved both numerically and analytically in section 7 for an initial state that exhibits a three-dimensional local structure. We find a solution which describes a series of $(3+1)$-dimensional de Sitter-like spacetimes with the Lorentzian signature which are bridged by 4 -dimensional Euclidean spaces. We show that the signature-changing dynamical phase transitions are caused by Lifshitz transitions in which the dispersion of the collective variables are inverted dynamically. The spacetime that emerges from a generic initial condition breaks the Lorentz symmetry. However, the Minkowski solution can be found with fine-tuning. We derive an effective theory that describes propagating modes, which are small fluctuations of the collective variables. We show that bi-local fields propagate in the spacetime determined from the saddle-point configuration, obeying local dynamics. In section 8 , we conclude with discussions on connections with the dS/CFT and AdS/CFT correspondences, and future directions.

Table 1 is a roadmap to the key concepts and results of the paper. 


\section{Kinematics}

\section{$2.1 \quad$ Hilbert space}

We consider an $M \times L$ real rectangular matrix, $\Phi^{A}{ }_{i}$ with $A=1,2, \ldots, M$ and $i=1,2, \ldots, L$ in the $M \gg L \gg 1$ limit. The full Hilbert space is spanned by $|\Phi\rangle \equiv \otimes_{i, A}\left|\Phi^{A}{ }_{i}\right\rangle$, where $\left|\Phi^{A}{ }_{i}\right\rangle$ is the eigenstate of $\hat{\Phi}_{i}^{A}$ with eigenvalue $\Phi^{A}{ }_{i}$. The inner product between basis states is given by

$$
\left\langle\Phi^{\prime} \mid \Phi\right\rangle=\prod_{i, A} \delta\left(\Phi_{i}^{\prime}{ }_{i}-\Phi_{i}^{A}\right)
$$

We note that the Fock space spanned by $\{|\Phi\rangle\}$ is infinite-dimensional for any $M>0$ and $L>0$ because $|\Phi\rangle$ and $\left|\Phi^{\prime}\right\rangle$ are orthogonal unless $\Phi=\Phi^{\prime}$. The conjugate momentum denoted as $\hat{\Pi}^{i}{ }_{A}$ satisfies the standard commutation relation, $\left[\hat{\Phi}_{i}^{A}, \hat{\Pi}^{j}{ }_{B}\right]=i \delta_{i}^{j} \delta_{B}^{A} . \hat{\Phi}(\hat{\Pi})$ represents the $M \times L(L \times M)$ operator valued matrix.

The row index $A$ is referred to as flavour index. In this paper, we consider a model that has the $\mathrm{O}(M)$ flavour symmetry generated by

$$
\hat{T}_{A B}=\frac{1}{2}\left(\hat{\Phi}_{A i} \hat{\Pi}_{B}^{i}-\hat{\Phi}_{B i} \hat{\Pi}_{A}^{i}\right),
$$

where the flavour indices are raised or lowered with the Euclidean metric: $\hat{\Phi}_{A i}=\hat{\Phi}_{i}{ }_{i}$. The flavour symmetry acts on $\Phi(\Pi)$ from the left (right) as

$$
\begin{aligned}
& e^{-i \operatorname{tr}\{\tilde{o} \hat{T}\}} \hat{\Phi} e^{i \operatorname{tr}\{\tilde{o} \hat{T}\}}=O \hat{\Phi}, \\
& e^{-i \operatorname{tr}\{\tilde{o} \hat{T}\}} \hat{\Pi} e^{i \operatorname{tr}\{\tilde{o} \hat{T}\}}=\hat{\Pi} O^{-1},
\end{aligned}
$$

where $\tilde{o}$ is an anti-symmetric matrix and $O=e^{-\tilde{o}} \in \mathrm{O}(M)$. General $\mathrm{O}(M)$ invariant operators can be constructed as composites of the following bi-linears,

$$
\hat{\Pi} \hat{\Phi}, \quad \hat{\Pi} \hat{\Pi}^{T}, \quad \hat{\Phi}^{T} \hat{\Phi},
$$

where $\hat{\Phi}^{T}\left(\hat{\Pi}^{T}\right)$ denotes the transpose of $\hat{\Phi}(\hat{\Pi})$. Products of operator valued matrices are defined in the usual way, e.g., $(\hat{\Pi} \hat{\Phi})^{i}{ }_{j}=\hat{\Pi}_{A}^{i} \hat{\Phi}^{A}{ }_{j}$. Henceforth, all repeated indices are understood to be summed over unless mentioned otherwise.

\section{$2.2 \quad$ Frame}

The column index $i$ is referred to as site index as it labels points of space in the model of gravity to be constructed. Once we identify $i$ as a site index, it is natural to write the total Hilbert space as a direct product of local Hilbert spaces as

$$
\mathbb{H}=\otimes_{i} \mathbb{H}_{i}
$$

where $\mathbb{H}_{i}$ is the local Hilbert space spanned by $\otimes_{A}\left|\Phi_{i}^{A}\right\rangle$. Such a decomposition of the total Hilbert space is called a frame. In a given frame, each site is associated with an infinite-dimensional local Hilbert space spanned by basis states, each of which is labeled 


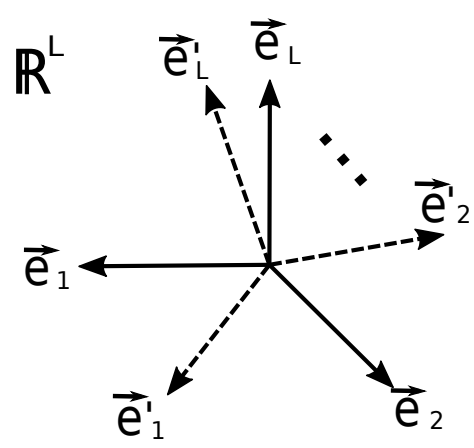

Figure 3. The total Hilbert space can be written as a direct product of local Hilbert spaces in a frame. A frame is given by $L$ linearly independent basis vectors that form an $L$-dimensional parallelepiped with the unit Euclidean volume in $\mathbb{R}^{L}$. Each basis vector determines a local Hilbert space. Under a special linear transformation, a frame can be rotated into another frame, which defines a new set of local Hilbert spaces.

by an $\mathrm{O}(M)$ vector. In the limit that both $M$ and $L$ are large, a manifold with any spatial dimension can emerge as will be shown in the next section. This is a key difference from other approaches to quantum gravity in which local Hilbert space is tailored for a specific space dimension.

The total Hilbert space can be decomposed in different frames. For example, one can use a different set of basis states that are related to the original basis states through

$$
\mid \Phi) \equiv|\tilde{\Phi}\rangle
$$

where $\tilde{\Phi}_{i}^{A}=g_{i}^{I} \Phi^{A}{ }_{I}$ and $g \in \mathrm{SL}(L, \mathbb{R})$. Eq. (2.6) should be understood as a change of basis in the infinite dimensional Fock space. The inner product is preserved because

$$
\left(\Phi \mid \Phi^{\prime}\right)=\prod_{A} \prod_{i} \delta\left(g_{i}^{I}\left(\Phi_{I}^{A}-\Phi_{I}^{\prime}{ }_{I}\right)\right)=\prod_{I, A} \delta\left(\Phi^{\prime}{ }_{I}-\Phi_{I}^{A}\right)
$$

where $\operatorname{det} g=1$ is used. This allows one to represent $\left.\mid \Phi)=\otimes_{A, I} \mid \Phi_{I}^{A}\right)$, where $\otimes_{A} \mid \Phi_{I}^{A}$ ) spans the local Hilbert space $\mathbb{H}_{I}^{\prime}$ at site $I$ in the rotated frame. The total Hilbert space can be written as a direct product of the local Hilbert spaces in the rotated frame as $\mathbb{H}=\otimes_{I} \mathbb{H}_{I}^{\prime}$.

A frame, denoted as $X$, is defined by a set of $L$ linearly independent row vectors in $\mathbb{R}^{L}: X=\left\{\vec{e}^{i} \in \mathbb{R}^{L} \mid V\left(\vec{e}^{1}, \vec{e}^{2}, \ldots, \vec{e}^{L}\right)=1, i=1,2, \ldots, L\right\}$, where $V\left(\vec{e}^{1}, \vec{e}^{2}, \ldots, \vec{e}^{L}\right)$ is the Euclidean volume of the parallelepiped formed by the $L$ vectors. This is illustrated in figure 3. If $X$ is a frame, $X_{g}=\left\{\vec{e}^{i} g \mid i=1,2, \ldots, L\right\}$ is also a frame for any $g \in \operatorname{SL}(L, \mathbb{R})$. A frame defines a set of local Hilbert spaces of which the total Hilbert is decomposed as a direct product. Associated with a frame, one can define a set of local observers: a local observer at site $i$ in frame $X$ has access to $\mathbb{H}_{i}^{X}$, where $\mathbb{H}_{i}^{X}$ is the local Hilbert space defined at site $i$ in frame $X$. The Hilbert space accessible to a local observer in one frame is comprised of linear superpositions of states accessible to multiple local observers in another frame. There is a priori no preferred frame and thus no preferred set of local observers. 


\subsection{Local structure}

In a given frame, one can define entanglement formed across local Hilbert spaces. In the presence of a local structure of entanglement, a spatial manifold can be defined from the pattern of entanglement. A state is defined to have a local structure in a frame if there exists a mapping from the sites to a Riemannian manifold such that the mutual information between any two sites decays exponentially in the proper distance between the images of the sites in the Riemannian manifold to the leading order in the proper distance (see figure 2). ${ }^{3}$ The dimension, topology and metric of the manifold are collective properties of a state. A state with a local structure can be regarded as a short-range entangled state with respect to the corresponding spatial manifold. For general states, local structure does not exist. The existence of local structure is a dynamical property that only a sub-set of states possess. Dimension, topology and geometry are order parameters that differentiate different local structures. Just as the vacuum expectation value of a field can be used an order parameter that characterizes symmetry of states in quantum field theories, dimension, topology and geometry in the present theory represent a set of coarse-grained data that characterize the macroscopic structure of quantum states. Dimension and topology form discrete order parameters, and two states with different dimensions or topologies can not be smoothly deformed to each other. On the other hand, geometry quantifies finer patterns of entanglement present across local Hilbert spaces.

In order to illustrate the idea, we consider a set of $\mathrm{O}(M)$ invariant states labeled by a collective variable,

$$
|t\rangle=\int d \Phi e^{i t^{i j} \Phi_{i}^{A} \Phi_{j}^{A}}|\Phi\rangle
$$

where $t^{i j}$ is a complex $L \times L$ collective variable. Here $\int d \Phi \equiv \prod_{i, A} \int_{-\infty}^{\infty} d \Phi^{A}{ }_{i}$. The state is normalizable as far as the eigenvalues of the $L \times L$ matrix, $t^{i j}$ lie within the upper half of the complex plane. If $t^{i j}$ is diagonal, $|t\rangle$ represents a direct product state with no entanglement. In order to see how off-diagonal elements of $t$ is related to inter-site entanglement, we compute the mutual information between two sites for eq. (2.8). For states that are close to the direct product state, the mutual information can be computed perturbatively in $\left|\frac{t^{i j}}{I m t^{i i}}\right| \ll 1$. An explicit calculation shows that the mutual information between sites $i$ and $j$ is given by [39]

$$
I_{i j}=2 M\left(-\ln \frac{\left|t^{i j}\right|^{2}}{4 \operatorname{Im} t^{i i} I m t^{j j}}+1\right) \frac{\left|t^{i j}\right|^{2}}{4 \operatorname{Im} t^{i i} I m t^{j j}}+\ldots
$$

to the leading order in $\frac{t^{i j}}{I m t^{i i}}$. Eq. (2.9) shows that $t^{i j}$ that connects sites $i$ and $j$ creates the mutual information between the sites to the lowest order. The ellipsis in eq. (2.9) represents the higher order mutual information formed through chains of link variables that connect $i$ and $j$ through other sites, $\sum_{n+m>0} \sum_{k_{1}, \ldots, k_{n}} \sum_{l_{1}, \ldots, l_{m}} \frac{t^{i k_{1}}\left(\prod_{a=1}^{n-1} t^{k_{a} k_{a+1}}\right) t^{k_{n} j} t^{j l_{1}}\left(\prod_{b=1}^{m-1} t^{l_{b} l_{b+1}}\right) t^{l_{m i}}}{t^{i i} t^{j j}\left(\prod_{a=1}^{n} t^{k_{a} k_{a}}\right)\left(\prod_{b=1}^{m} t^{l b_{b}}\right)}$.

\footnotetext{
${ }^{3}$ For states that have local structures, the von Neumann entanglement entropy of a subset of sites scales with the boundary of the corresponding subregion in the Riemannian manifold. One can alternatively use this as a definition of local structure [34].
} 
Sites that are not directed connected by a non-zero collective variable are entangled via multiple legs of the bi-local collective variables. The off-diagonal elements of the collective variable describe 'bonds' that create inter-site entanglement, where the strength of the bond between sites $i$ and $j$ is proportional to the magnitude of $t^{i j}$. If the short-ranged entanglement bonds form a regular lattice (similar to the way a lattice is formed by chemical bonds in solids), the corresponding state has a local structure that exhibits a manifold with a well-defined dimension and topology. We will later see how the emergent geometry is determined from the collective variables as well. Intuitively, the geometry is determined such that the proper distance between two points gets smaller if the two points are connected by stronger entanglement bonds (larger $t^{i j}$ ).

The same set of sites can exhibit manifolds with different dimensions, topologies and geometries, depending on the pattern of entanglement. Let us consider a few examples of states with local structures. As a first example, we consider the state in eq. (2.8) with

$$
t^{i j}=i\left(\delta_{i j}+\epsilon \delta_{|i-j|, 1}\right)
$$

for $\epsilon \ll 1$. In this state, nearest neighbour entanglement bonds form an open chain. For this state, the local structure is manifest in the one-dimensional coordinate system,

$$
r_{j}=j .
$$

The emergent one-dimensional space has the topology of a line segment, $[0,1]$. In particular, the physical distance between the first site and the last site is very far due to the weak entanglement. If one creates a direct entanglement bond between sites 1 and $L$ by adding $t^{1 L}=i \epsilon$ to eq. (2.10), the two sites become neighbours. As a consequence, the topology of the space changes to $S^{1}$.

As a second example, let us consider the collective variable given by

$$
t^{i j}=i\left(\delta_{i j}+\epsilon \delta \sqrt{\left(r_{i}^{1}-r_{j}^{1}\right)^{2}+\left(r_{i}^{2}-r_{j}^{2}\right)^{2}}, 1\right),
$$

where

$$
\left(r_{j}^{1}, r_{j}^{2}\right)=\left(j \bmod \sqrt{L},\left\|\frac{j-1}{\sqrt{L}}\right\|+1\right)
$$

with $\|x\|$ being the largest integer equal to or smaller than $x$. In this state, the entanglement bonds form a square lattice. It exhibits a two-dimensional local structure with the topology of a disk. Eq. (2.13) is a natural coordinate system in which the local structure is manifest. These are illustrated in figure 4. These examples illustrate the fact that dimension and topology of space are nothing but collective variables of the underlying matrix. In section 7 , we will show how the geometry is determined from the collective variables.

The existence of local structure depends on the choice of frame. Under a change of frame, the collective variable is transformed as $t^{\prime I J}=g_{i}^{I} g_{j}^{J} t^{i j}$, where $g \in \mathrm{SL}(L, \mathbb{R})$. Even if a state exhibits a local entanglement structure in one frame, it does not have a local structure in another frame if the latter is related to the former through a transformation that is non-local with respect to the locality defined in one frame. Generic states with bonds that form a global network do not exhibit a local structure. 


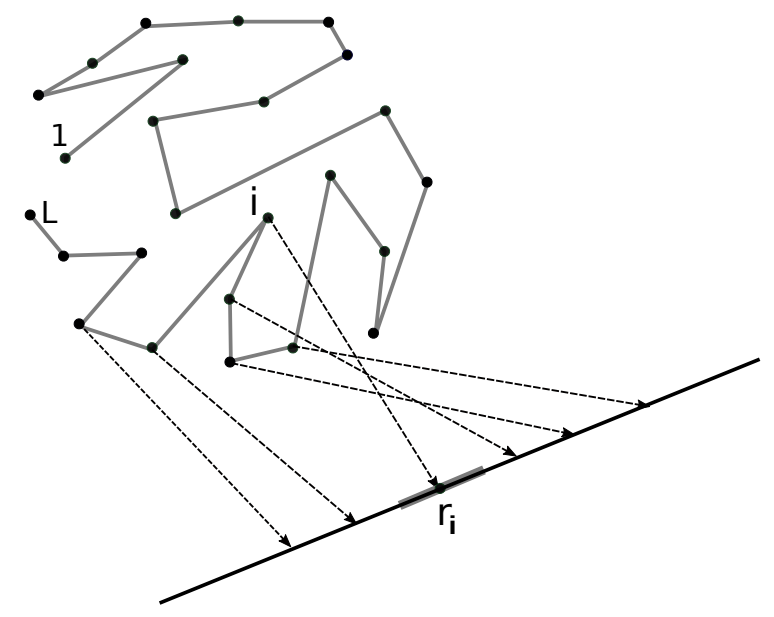

(a)

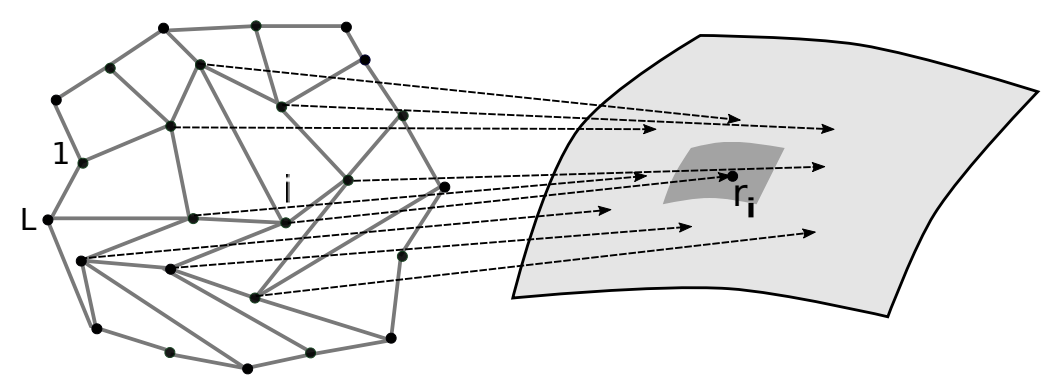

(b)

Figure 4. The panels (a) and (b) represent states in eq. (2.8) with local structures given by eq. (2.10) and eq. (2.12), respectively. In the figures on the left, the dots represent sites in a frame, and the links represent entanglement bonds formed by bi-local collective variable, $t^{i j}$. The dashed lines represent mappings from sites to manifolds. In the figures on the right, the grey area around $r_{i}$ denotes the region of coordinate volume $V_{i}$ assigned to site $i$ in the manifold. The dimension and topology of the manifold in which the local structure is manifest are determined from the pattern of entanglement bonds. In the large $L$ limit, the proper distances between sites 1 and $L$ in states (a) and (b) scale as $\mathrm{O}(L)$ and $\mathrm{O}(1)$ respectively due to different local entanglement structures.

\section{Gauge symmetry}

In this section, we construct the generators of the gauge symmetry that generalizes the spacetime diffeomorphism of the general relativity. Since we are going to use the Hamiltonian formalism, we first review the Hamiltonian formulation of the general relativity.

\subsection{Review of the Hamiltonian formalism of the general relativity}

In $(3+1)$ dimensions, the action of the general relativity can be written as [40]

$$
S=\int d \tau d^{3} r\left[\pi^{\mu \nu} \partial_{\tau} g_{\mu \nu}-\xi^{\mu}(r) \mathscr{P}_{\mu}(r)-\theta(r) \mathscr{H}(r)\right] .
$$

Here a four-dimensional spacetime is decomposed into a stack of three-dimensional spatial manifolds that are labeled by coordinate time $\tau$. A point within each time slice 
is labeled by $r . g_{\mu \nu}$ and $\pi^{\mu \nu}$ with $\mu, \nu=1,2,3$ are the spatial metric and its conjugate variable, respectively. The symplectic form in eq. (3.1) defines the Poisson bracket, $\left\{g_{\mu \nu}(r), \pi^{\rho \sigma}\left(r^{\prime}\right)\right\}_{\mathrm{PB}}=\delta_{\mu \nu}^{\rho \sigma} \delta\left(r-r^{\prime}\right)$, where $\delta_{\mu \nu}^{\rho \sigma}=\frac{1}{2}\left(\delta_{\mu}^{\rho} \delta_{\nu}^{\sigma}+\delta_{\nu}^{\rho} \delta_{\mu}^{\sigma}\right) . \mathscr{P}_{\mu}(r)$ is the momentum density that generates spatial diffeomorphism within a spatial manifold. $\xi^{\mu}(r)$ is the shift that specifies an infinitesimal spatial diffeomorphism. $\mathscr{H}(r)$ is the Hamiltonian density that generates local time translation. $\theta(r)$ is the lapse that determines the position dependent time translation. The shift and the lapse can be chosen arbitrarily. Consequently, the momentum and Hamiltonian,

$$
\begin{aligned}
P[\vec{\xi}] & =\int d^{3} r \xi^{\mu}(r) \mathscr{P}_{\mu}(r), \\
H[\theta] & =\int d^{3} r \theta(r) \mathscr{H}(r)
\end{aligned}
$$

become constraints. The key property of the general relativity is that the entire dynamics is generated by the constraints that satisfy the hypersurface deformation algebra [41],

$$
\begin{gathered}
\left\{P\left[\vec{\xi}_{1}\right], P\left[\vec{\xi}_{2}\right]\right\}_{\mathrm{PB}}=P\left[\mathscr{L}_{\vec{\xi}_{1}} \vec{\xi}_{2}\right], \\
\{P[\vec{\xi}], H[\theta]\}_{\mathrm{PB}}=H\left[\mathscr{L}_{\vec{\xi}} \theta\right], \\
\left\{H\left[\theta_{1}\right], H\left[\theta_{2}\right]\right\}_{\mathrm{PB}}=P\left[\vec{\xi}_{\theta_{1}, \theta_{2}}\right] .
\end{gathered}
$$

Here $\mathscr{L}_{\vec{\xi}}$ represents the Lie derivative with respect to the vector field $\vec{\xi}$ and

$$
\xi_{\theta_{1}, \theta_{2}}^{\mu}=-\delta g^{\mu \nu}\left(\theta_{1} \nabla_{\nu} \theta_{2}-\theta_{2} \nabla_{\nu} \theta_{1}\right) .
$$

The signature of spacetime is chosen to be $(\mathcal{S},+,+,+)$. For a Lorentzian (Euclidean) spacetime, $\mathcal{S}=-1(+1)$. Eq. (3.3) and eq. (3.4) denote the fact that $\mathscr{P}_{\mu}(r)$ and $\mathscr{H}(r)$ transform as a vector density and a scalar density respectively under a spatial diffeomorphism. These two are purely kinematic. On the other hand, eq. (3.5) implies that two successive infinitesimal local time translations performed in different orders are related to each other through a spatial diffeomorphism. Under an infinitesimal time translation generated by lapse $\theta_{1}$, a general phase space function $f(g, \pi)$ evolves into $f+\epsilon\left\{f, H\left[\theta_{1}\right]\right\}_{\mathrm{PB}}+$ $\frac{\epsilon^{2}}{2}\left\{\left\{f, H\left[\theta_{1}\right]\right\}_{\mathrm{PB}}, H\left[\theta_{1}\right]\right\}_{\mathrm{PB}}$ to the second order in $\epsilon$. A consecutive time evolution generated by lapse $\theta_{2}$ gives $f+\epsilon\left\{f, H\left[\theta_{1}\right]\right\}_{\mathrm{PB}}+\epsilon\left\{f, H\left[\theta_{2}\right]\right\}_{\mathrm{PB}}+\frac{\epsilon^{2}}{2}\left\{\left\{f, H\left[\theta_{1}\right]\right\}_{\mathrm{PB}}, H\left[\theta_{1}\right]\right\}_{\mathrm{PB}}+$ $\frac{\epsilon^{2}}{2}\left\{\left\{f, H\left[\theta_{2}\right]\right\}_{\mathrm{PB}}, H\left[\theta_{2}\right]\right\}_{\mathrm{PB}}+\epsilon^{2}\left\{\left\{f, H\left[\theta_{1}\right]\right\}_{\mathrm{PB}}, H\left[\theta_{2}\right]\right\}_{\mathrm{PB}}$. The time translations applied in the opposite order results in a different outcome, $f+\epsilon\left\{f, H\left[\theta_{2}\right]\right\}_{\mathrm{PB}}+\epsilon\left\{f, H\left[\theta_{1}\right]\right\}_{\mathrm{PB}}+$ $\frac{\epsilon^{2}}{2}\left\{\left\{f, H\left[\theta_{2}\right]\right\}_{\mathrm{PB}}, H\left[\theta_{2}\right]\right\}_{\mathrm{PB}}+\frac{\epsilon^{2}}{2}\left\{\left\{f, H\left[\theta_{1}\right]\right\}_{\mathrm{PB}}, H\left[\theta_{1}\right]\right\}_{\mathrm{PB}}+\epsilon^{2}\left\{\left\{f, H\left[\theta_{2}\right]\right\}_{\mathrm{PB}}, H\left[\theta_{1}\right]\right\}_{\mathrm{PB}}$. The discrepancy between the two is generated by the shift given in eq. (3.6) to the order of $\epsilon^{2}$ due to the Jacobi identity. This relation has some dynamical information because eq. (3.6) depends on the spatial metric and the signature of spacetime. In other words, the discrepancy is compensated by different shifts in different states. This will be important in identifying the emergent metric degree of freedom in our theory later in this paper.

The Hamiltonian and momentum form the first-class constraint algebra classically, which is crucial to guarantee that the constraints are preserved under the evolution generated by the constraints themselves. The constraint algebra largely fixes the form of the 
Einstein-Hilbert action. Up to two derivative order, the Einstein-Hilbert action is the only theory that satisfies eqs. (3.3), (3.4) and (3.5) [41, 42]. In quantum gravity, the constraints are to be promoted to operators that satisfy a first-class operator algebra. The challenge is to regularize the constraints in a way that they satisfy a first-class algebra at the quantum level which is reduced to eq. (3.3)-(3.5) in the classical limit.

In the following two subsections, we construct momentum and Hamiltonian constraints that generate generalized spacetime diffeomorphism in the absence of manifold with fixed dimension and topology. We impose the $\mathrm{O}(M)$ flavour symmetry, and the constraints are built out of the bi-linears in eq. (2.4).

\subsection{Momentum constraint}

Because dimension and topology of spatial manifold are not fixed, spatial diffeomorphism needs to be generalized to a group that includes diffeomorphism in any dimension in the limit that $L$ is large. For any pair of sites $i$ and $j$, there should exist a generator that maps $i$ to $j$ because there are states with local structures in which the two sites are close to each other. The desired gauge group is the special linear group $(\operatorname{SL}(L, \mathbb{R}))$ introduced in section 2.2 that generates rotations of frame.

The first operator in eq. (2.4) generates the general linear transformation. The Hermitian generator of $\mathrm{GL}(L, \mathbb{R})$ is given by

$$
\hat{\mathbf{G}}_{j}^{i}=\frac{1}{2}\left(\hat{\Pi}_{A}^{i} \hat{\Phi}_{j}^{A}+\hat{\Phi}_{j}^{A} \hat{\Pi}_{A}^{i}\right),
$$

where $i, j=1,2, \ldots, L$. The $\mathrm{GL}(L, \mathbb{R})$ generators can be decomposed into $\left(L^{2}-1\right)$ generators for $\mathrm{SL}(L, \mathbb{R})$,

$$
\hat{G}^{i}{ }_{j}=\hat{\mathbf{G}}^{i}{ }_{j}-\frac{1}{L} \hat{\mathbf{G}}^{k}{ }_{k}{ }^{i}{ }_{j},
$$

and one for the global dilatation,

$$
\hat{G}_{0}=\frac{1}{L} \hat{\mathbf{G}}_{k}^{k} \text {. }
$$

Because the inner product is preserved under $\operatorname{SL}(L, \mathbb{R})$ as is shown in eq. (2.7), we pick $\operatorname{SL}(L, \mathbb{R})$ as the gauge group that generalizes the spatial diffeomorphism. $\operatorname{SL}(L, \mathbb{R})$ generators can be written as

$$
\hat{G}_{y}=\operatorname{tr}\{\hat{G} y\}
$$

where $y$ is a traceless $L \times L$ real matrix. The $\operatorname{SL}(L, \mathbb{R})$ transformations act on $\Phi(\Pi)$ from the right (left) as

$$
\begin{aligned}
& e^{-i \hat{G}_{y}} \hat{\Phi} e^{i \hat{G}_{y}}=\hat{\Phi} g_{y}, \\
& e^{-i \hat{G}_{y}} \hat{\Pi} e^{i \hat{G}_{y}}=g_{y}^{-1} \hat{\Pi},
\end{aligned}
$$

where $g_{y}=e^{-y} \in \mathrm{SL}(L, \mathbb{R})$. Under $\mathrm{SL}(L, \mathbb{R}), \Phi(\Pi)$ transforms covariantly (contravariantly). 
Now we examine how $\mathrm{SL}(L, \mathbb{R})$ acts on the matrix in states which have local structures. In the presence of a local structure, one can define a manifold into which sites are embedded. Let $r_{i}$ represent the point in the spatial manifold associated with site $i$. The matrix $\Phi^{A}{ }_{i}$ is then viewed as a field $\Phi^{A}\left(r_{i}\right)$ defined at position $r_{i}$. For an infinitesimal $\operatorname{SL}(L, \mathbb{R})$ transformation with $g_{y}=e^{-\epsilon y}$ in eq. (3.11), the field transforms as

$$
\Phi^{\prime A}\left(r_{i}\right)=\Phi^{A}\left(r_{i}\right)-\epsilon \sum_{j} \Phi^{A}\left(r_{j}\right) y_{i}^{j} .
$$

Let us consider field configurations that change slowly on the manifold in the continuum limit with $L \gg 1$. In this case, a gradient expansion can be used to write $\Phi^{A}\left(r_{j}\right)=$ $\Phi^{A}\left(r_{i}\right)+\partial_{\mu} \Phi^{A}\left(r_{i}\right)\left(r_{j}^{\mu}-r_{i}^{\mu}\right)+\ldots,{ }^{4}$ and eq. (3.12) becomes

$$
\Phi^{\prime} A\left(r_{i}\right)-\Phi^{A}\left(r_{i}\right)=-\epsilon \zeta_{y}\left(r_{i}\right) \Phi^{A}\left(r_{i}\right)-\epsilon \xi_{y}^{\mu}\left(r_{i}\right) \partial_{\mu} \Phi^{A}\left(r_{i}\right)+\ldots
$$

Here

$$
\begin{aligned}
\zeta_{y}\left(r_{i}\right) & =\sum_{j} y_{i}^{j}, \\
\xi_{y}^{\mu}\left(r_{i}\right) & =\sum_{j} y_{i}^{j}{ }_{i}\left(r_{j}^{\mu}-r_{i}^{\mu}\right)
\end{aligned}
$$

represents a scalar and a vector fields, respectively, associated with $y$. In eq. (3.13), ... represent higher derivative terms. The scalar field determines the position dependent rescaling of the field (Weyl transformation) [43]. The vector field describes spatial diffeomorphism. To the first derivative order, eq. (3.13) is precisely how a scalar field transforms under the Weyl transformation and the spatial diffeomorphism. While the former is an internal gauge symmetry, the latter is defined with reference to the manifold associated with the state. We note that the Weyl symmetry and the spatial diffeomorphism is a part of the larger gauge symmetry generated by $\operatorname{SL}(L, \mathbb{R})$. The generalized momentum constraint includes other gauge transformations associated with the higher derivative terms in eq. (3.13). Some of the extra gauge symmetry act non-locally with respect to the manifold selected by a state. They generate smooth diffeomorphism in different manifolds associated with states with different local structures. Namely, smooth diffeomorphisms acting locally in one manifold act non-locally in another manifold with different dimension and topology. Because there is no pre-determined manifold, the gauge symmetry should include all of them. The presence of extra gauge symmetry also plays an important role in determining the physical degrees of freedom of the theory, as will be discussed in section 4.4. We call $\hat{G}$ and $y$ the momentum constraint and the shift tensor, respectively.

A few remarks are in order. First, the diffeomorphism induced by $\operatorname{SL}(L, \mathbb{R})$ is an active transformation. Eq. (3.13) shows how the field is actively 'dragged' under $\operatorname{SL}(L, \mathbb{R})$ in a fixed coordinate system. Second, $\mathrm{SL}(L, \mathbb{R})$ includes smooth diffeomorphism of any dimension in the large $L$ limit. Once a $D$-dimensional coordinate system is chosen by

\footnotetext{
${ }^{4}$ One can view this as the defining expression of the gradient in the limit that $\Phi^{A}(r)$ changes slowly in space.
} 
the local structure of a state, there exists a set of shift tensors that generate general diffeomorphism in $D$-dimensional. For example, the state in eq. (2.8) with eq. (2.10) has the one-dimensional local structure. The shift tensor given by

$$
y_{i}^{j}=\frac{\xi_{i}}{2}\left(\delta_{j, i+1}-\delta_{j, i-1}\right)
$$

gives rise to a vector field $\xi\left(r_{i}\right)=\xi_{i}$ in the coordinate system given by eq. (2.11). This generates an one-dimensional diffeomorphism in the continuum limit. The state with eq. (2.12) has a two-dimensional local structure which is manifest in the coordinate system given by eq. (2.13). The shift tensor,

$$
y_{i}^{j}=\frac{\xi_{i}^{1}}{2}\left(\delta_{r_{j}^{1}, r_{i}^{1}+1}-\delta_{r_{j}^{1}, r_{i}^{1}-1}\right) \delta_{r_{j}^{2}, r_{i}^{2}}+\frac{\xi_{i}^{2}}{2}\left(\delta_{r_{j}^{2}, r_{i}^{2}+1}-\delta_{r_{j}^{2}, r_{i}^{2}-1}\right) \delta_{r_{j}^{1}, r_{i}^{1}}
$$

gives rise to a two-dimensional diffeomorphism generated by the vector field $\xi^{\mu}\left(r_{i}\right)=$ $\left(\xi_{i}^{1}, \xi_{i}^{2}\right)$ on the two-dimensional manifold in the continuum limit. These examples show that $\operatorname{SL}(L, \mathbb{R})$ include general diffeomorphism in arbitrary dimensions. Third, $D$-dimensional diffeomorphisms act locally only in states with a $D$-dimensional local structure. In general, a $\operatorname{SL}(L, \mathbb{R})$ transformation that generates a $D$-dimensional diffeomorphism acts as a non-local transformation in states with local structures with different dimensions. For example, the shift tensor in eq. (3.17) generates a non-local transformation in the onedimensional manifold given by eq. (2.11), while it generates a local diffeomorphism in the two-dimensional manifold in eq. (2.13). A transformation that maps site 1 to site $L$ is quasi-local in figure 4(b) but non-local in figure 4(a) in the continuum limit.

\subsection{Hamiltonian constraint}

Having identified the generator for the spatial diffeomorphism, we now construct the Hamiltonian density. In the general relativity, the Hamiltonian density forms a representation of scalar density under spatial diffeomorphism. Each element in the representation generates one of many-fingered time translations. In the present theory, Hamiltonian should form a representation of $\operatorname{SL}(L, \mathbb{R})$. Given that the column indices of the matrix play the role of sites in the present theory, one may expect that Hamiltonian density forms a vectorial representation of $\operatorname{SL}(L, \mathbb{R})$. However, there is no $\mathrm{O}(M)$ invariant operator that forms a vectorial representation. This is because all $\mathrm{O}(M)$ invariant operators should be constructed from the bi-linears in eq. (2.4). To be concrete, we first consider the usual $\mathrm{O}(M)$ invariant kinetic operator localized at a site, $h^{i i}=\sum_{A} \hat{\Pi}_{A}^{i} \hat{\Pi}^{i}{ }_{A}$ as a candidate for the Hamiltonian density (here, $i$ is not summed over). The problem is that the set of $\left\{h^{i i} \mid i=1,2, \ldots, L\right\}$ does not form a representation of $\operatorname{SL}(L, \mathbb{R})$. Under $\mathrm{SL}(L, \mathbb{R})$, the ultra-local kinetic term is transformed to $e^{-i \hat{G}_{y}} h^{i i} e^{i \hat{G}_{y}}=\sum_{k, l} \sum_{A}\left(g_{y}^{-1}\right)_{k}^{i}\left(g_{y}^{-1}\right)_{l}^{i} h^{k l}$, which is not ultra-local any more. This underlines the fact that the notion of locality is frame dependent. Even if the kinetic term is local in one frame, it is generally not in other frames. In order to construct a kinetic term that forms a representation of $\operatorname{SL}(L, \mathbb{R})$, we need to include the full $L \times L$ matrix, $\hat{\Pi} \hat{\Pi}^{T}$ which forms a rank 2 contravariant symmetric representation of $\operatorname{SL}(L, \mathbb{R})$. The most general kinetic operator is labeled by a rank 2 symmetric tensor $v$ as

$$
\hat{h}_{1, v}=\operatorname{tr}\left\{\hat{\Pi} \hat{\Pi}^{T} v\right\} \text {. }
$$


It is reminded that the trace in eq. (3.18) sums over both the flavour and the site indices. In the component form, eq. (3.18) reads $\sum_{A} \sum_{i, j} \hat{\Pi}_{A}^{i} \hat{\Pi}^{j}{ }_{A} v_{j i}$. Under $\operatorname{SL}(L, \mathbb{R}), v$ transforms as $v \rightarrow g_{y}^{-1 T} v g_{y}^{-1}$. The set of $\hat{h}_{1, v}$ with symmetric $v$ forms a representation under $\operatorname{SL}(L, \mathbb{R})$ . We refer to $v$ as the lapse tensor as it plays the role of the lapse function in the general relativity. Before we discuss the meaning of the lapse tensor further, let us complete the construction of the Hamiltonian. We need to add a hopping operator that becomes gradient term $\left(|\nabla \Phi|^{2}\right)$ in the continuum limit. As a part of the Hamiltonian that is added to eq. (3.18), it should also form a rank 2 symmetric contravariant tensorial representation of $\operatorname{SL}(L, \mathbb{R})$. The bilinear, $\Phi_{i}^{A} \Phi_{j}^{A}$ is a candidate of the hopping term, but it is a covariant tensor not a contravariant tensor. In order to convert it into a contravariant tensor, the site indices should be raised with $\hat{\Pi} \hat{\Pi}^{T}$. The minimal hopping term that includes $\Phi_{i}^{A} \Phi_{j}^{A}$ and transforms in the desired representation is

$$
\hat{h}_{2, v}=\frac{1}{M^{2}} \operatorname{tr}\left\{\hat{\Pi} \hat{\Pi}^{T} \hat{\Phi}^{T} \hat{\Phi} \hat{\Pi} \hat{\Pi}^{T} v\right\} .
$$

The factor of $M^{-2}$ is introduced in eq. (3.19) to make sure that both $\hat{h}_{1, v}$ and $\hat{h}_{2, v}$ scale as $\mathrm{O}(M)$ in the large $M$ limit. We combine $\hat{h}_{1, v}$ and $\hat{h}_{2, v}$ to write the Hamiltonian as $\hat{H}_{v}=\tilde{\alpha}_{1} \hat{h}_{1, v}+\tilde{\alpha}_{2} \hat{h}_{2, v}$, where $\tilde{\alpha}_{1}$ and $\tilde{\alpha}_{2}$ are dimensionless parameters. We choose $\tilde{\alpha}_{2}>0$ such that the Hamiltonian is bounded from below for large $\Pi$. Furthermore, $\tilde{\alpha}_{1}<0$ is chosen so that the space of configurations that satisfy the constraint $\hat{H}_{v}=0$ is nontrivial in the classical limit. ${ }^{5}$ Without loss of generality, one can set $\tilde{\alpha}_{1}=-1$. The full Hamiltonian with lapse tensor $v$ is written as

$$
\hat{H}_{v}=\operatorname{tr}\left\{\left(-\hat{\Pi} \hat{\Pi}^{T}+\frac{\tilde{\alpha}}{M^{2}} \hat{\Pi} \hat{\Pi}^{T} \hat{\Phi}^{T} \hat{\Phi} \hat{\Pi} \hat{\Pi}^{T}\right) v\right\} .
$$

In order to understand the meaning of the Hamiltonian, it is convenient to go to the frame in which the lapse tensor is diagonal. A non-singular lapse tensor can be written as

$$
v=n_{v} g_{v}^{T} S_{v} g_{v}
$$

where $n_{v}$ is a positive number, $g_{v} \in \mathrm{SL}(L, \mathbb{R})$ and $S_{v}$ is a diagonal matrix whose elements are either 1 or -1 . See appendix A for the proof. Then, eq. (3.20) can be written as

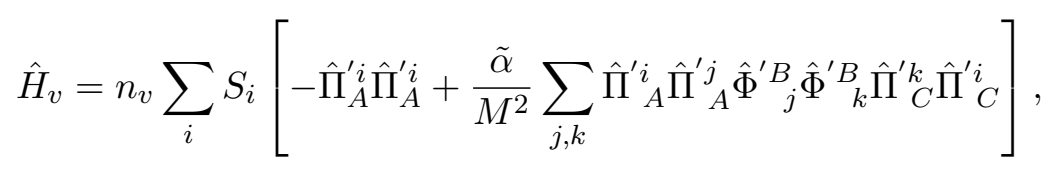

where $\hat{\Phi}^{\prime}=\hat{\Phi} g_{v}^{-1}$ and $\hat{\Pi}^{\prime}=g_{v} \hat{\Pi}$. Henceforth, let us omit the prime signs. Here $S_{i}$ determines the direction of local time evolution at each site $i$. The first term in the bracket of eq. (3.22) is an ultra-local kinetic term. The second term describes a hopping process, where a particle jumps from sites $j$ to $k$ (and vice versa) with a hopping amplitude proportional to $\left(\hat{\Pi}_{A}^{j} \hat{\Pi}_{A}^{i}\right)\left(\hat{\Pi}_{C}^{i} \hat{\Pi}_{C}^{k}\right)$. The hopping amplitude between two sites is given by the amplitudes of bi-local operators that connect the two sites through a third site $i$ as is shown in figure 5 . In the large $M$ limit, the bi-linear operator $\left(\hat{\Pi}_{A}^{j} \hat{\Pi}_{A}^{i}\right)$ has a well-defined

\footnotetext{
${ }^{5}$ Both $\hat{\Pi} \hat{\Pi}^{T}$ and $\hat{\Phi}^{T} \hat{\Phi}$ are non-negative matrices. If both $\tilde{\alpha}_{1}$ and $\tilde{\alpha}_{2}$ are positive, the only configuration that satisfies $\hat{H}_{v}=0$ is $\hat{\Pi} \hat{\Pi}^{T}=\hat{\Phi}^{T} \hat{\Phi}=0$ classically.
} 


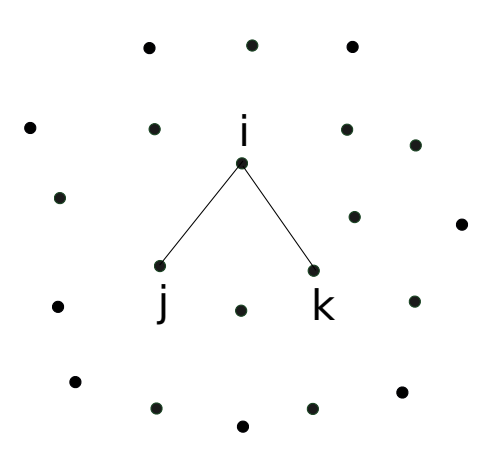

Figure 5. The local Hamiltonian density defined at site $i$ in eq. (3.22) includes hoppings between sites $j$ and $k$ which are connected through $i$ via two entanglement bonds. The hopping amplitude is proportional to the product of $\left(\hat{\Pi} \hat{\Pi}^{T}\right)^{i j}$ and $\left(\hat{\Pi} \hat{\Pi}^{T}\right)^{k i}$.

expectation value for $\mathrm{O}(M)$ invariant states. For the state in eq. (2.8), the expectation value of the bi-local operator is given by the collective variable

$$
\frac{1}{M} \frac{\left\langle t\left|\left(\hat{\Pi}_{A}^{j} \hat{\Pi}_{A}^{i}\right)\right| t\right\rangle}{\langle t \mid t\rangle}=-2 i\left(t^{-1}-t^{*-1}\right)_{i j}^{-1} .
$$

The collective variable $t^{i j}$ in turn controls the mutual information between sites $i$ and $j$ through eq. (2.9). Therefore, eq. (3.22) describes a relatively local kinetic term whose hopping amplitude is determined from the entanglement present between sites [25, 34]. Because entanglement is state dependent, so are the hopping amplitudes and the graph that is formed by the network of hopping amplitudes. Since the underlying matrix is dynamical, the emergent manifold that is formed by the entanglement bonds is fully dynamical. In a globally entangled state, the Hamiltonian acts as a non-local Hamiltonian with global hoppings. In a state with a local structure, the Hamiltonian acts as a local Hamiltonian in the corresponding dimension set by the local structure. ${ }^{6}$ For this reason, the dimension, topology and geometry are all dynamical in this theory. ${ }^{7}$

For each choice of the lapse tensor, the Hamiltonian is relatively local in the frame in which the lapse tensor is diagonal. The gauge freedom in the choice of lapse tensor includes not only the freedom to choose site dependent speed of time evolution in a given frame ${ }^{8}$ but also the freedom to rotate the frame in which the lapse tensor is diagonal. The space of lapse tensors in the present theory is much larger than that of lapse functions in the general relativity. In the general relativity, the rate of local time flow can be chosen independently at each site. In the presence of $L$ sites, this would give rise to $L$ independent directions of multi-fingered time evolutions. In the present theory, there are $\frac{L(L+1)}{2}$ independent parameters in the lapse tensor. The extra $\frac{L(L-1)}{2}$ off-diagonal elements come from the

\footnotetext{
${ }^{6}$ We note that this is different from the notion of relative locality introduced in ref. [44].

${ }^{7}$ The relatively local hopping term in eq. (3.19) is different from the one considered in [25] in its detail form. The difference is caused by the fact that we are imposing the condition that the Hamiltonian should form a representation under the generalized spatial diffeomorphism group.

${ }^{8}$ This freedom is actually a part of redefining the frame itself in which the the relative magnitudes of the $L$ vectors that form a frame is rescaled without rotating the directions of the vectors.
} 


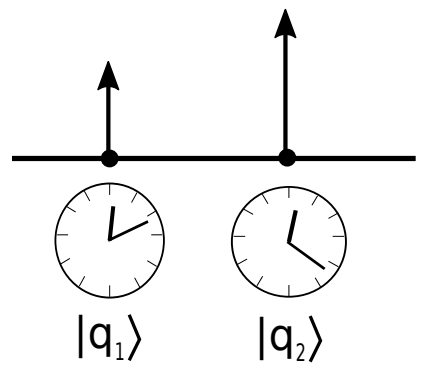

(a)

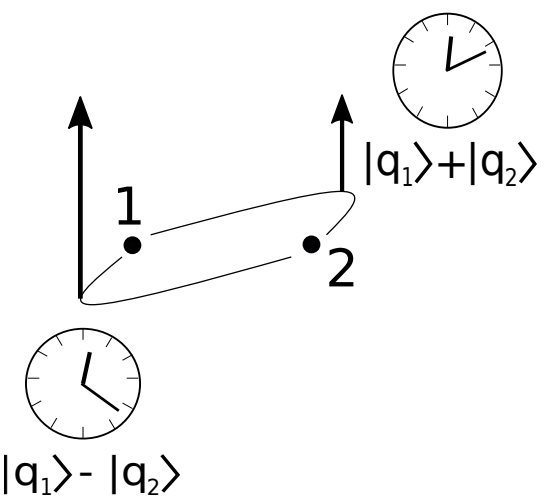

(b)

Figure 6. (a) In the general relativity, one can evolve a state defined on a Cauchy surface such that clocks at different locations in space run at different rates. This freedom of choosing space dependent lapse of time gives rise to the multi-fingered time evolutions. In the figure, $\left|q_{1}\right\rangle$ and $\left|q_{2}\right\rangle$ represent the clock degrees of freedom localized at sites 1 and 2 respectively. (b) In the present theory, there is a larger gauge freedom. One not only has a freedom to choose different rates for a given set of clocks, but also has a freedom to construct a whole different set of clocks out of degrees of freedom defined at multiple sites through linear superpositions. This extra freedom is encoded in the off-diagonal elements of the lapse tensor that rotates frame in eq. (3.21). In priori, there is no preferred frame in which the clocks are defined. In order to define a time evolution in a meaningful way, one has to define a set of clocks out of physical degrees of freedom within the theory and describe evolution of other degrees of freedom relative to the local evolution of the physical clocks.

freedom to rotate frames. As a result, the notion of the many-fingered time evolutions is generalized to a greater extent. This is illustrated in figure 6 .

In the general relativity, one can choose any lapse function to evolve a state defined on a time slice to a next time slice. In the presence of physical degrees of freedom which can be used to construct a set of local clocks, one can define a lapse function with respect to the clocks. For example, one can use radioactive atoms distributed over space as local clocks, and choose lapse such that the density of decayed atoms follow a specific profile in each time slices. In the present theory, one can follow time evolution in any frame by choosing general lapse tensor with off-diagonal elements. This gives rise to a larger gauge freedom. In the presence of physical degrees of freedom out of which a set of clocks can be constructed, one can choose a frame in which those clocks are local. If those clocks are initially unentangled, the lapse tensor that is diagonal in that frame evolves the clocks independently to the leading order in $M$. The time evolution of the remaining degrees of freedom generated by this Hamiltonian describes their evolution relative to the set of local clocks that tick independently. The relative motion of the other degrees of freedom measured against a chosen set of local clocks is the gauge invariant prediction of the theory. An example will be discussed in section 4.2.

\subsection{First-class constraint algebra}

The momentum and Hamiltonian constraints in eqs. (3.10) and (3.20) should be compared with $P[\vec{\xi}]$ and $H[\theta]$ respectively in eq. (3.2). $y$ and $v$ in the present theory play the 
role of $\xi^{\mu}(r)$ and $\theta(r)$ of the general relativity. Just as $\xi^{\mu}(r)$ and $\theta(r)$ are the Lagrangian multipliers that enforce $\mathscr{P}_{\mu}(r)|\Psi\rangle=0$ and $\mathscr{H}(r)|\Psi\rangle=0$ for a gauge invariant state at every point $r, y_{i}{ }_{i}$ and $v_{i j}$ enforce $\hat{G}^{i}{ }_{j}|\Psi\rangle=0$ and $\left(-\hat{\Pi} \hat{\Pi}^{T}+\frac{\tilde{\alpha}}{M^{2}} \hat{\Pi} \hat{\Pi}^{T} \hat{\Phi}^{T} \hat{\Phi} \hat{\Pi} \hat{\Pi}^{T}\right)^{i j}|\Psi\rangle=0$ for every $i$ and $j$, where $|\Psi\rangle$ is a gauge invariant state of the matrix $\Phi$.

In order for the momentum and Hamiltonian constraints to generate consistent gauge transformations of quantum gravity, they should satisfy a first-class constraint algebra. In this section, we check the algebra that momentum and Hamiltonian satisfy. The commutator between an operator and $\operatorname{SL}(L, \mathbb{R})$ generators is fixed by the representation that the operator forms under $\operatorname{SL}(L, \mathbb{R})$. $\Phi$ and $\Pi$ form the covariant and contravariant vectorial representations respectively as is shown in eq. (3.11). $\hat{G}$ and $\hat{H}$ are rank 2 mixed tensor and contravariant tensors respectively. This fixes their commutators with $\hat{G}$ to be

$$
\begin{aligned}
& {\left[\hat{G}_{x}, \hat{G}_{y}\right]=i \hat{G}_{(y x-x y)},} \\
& {\left[\hat{G}_{x}, \hat{H}_{v}\right]=i \hat{H}_{v x+x^{T} v} .}
\end{aligned}
$$

The commutator between Hamiltonians is more complicated. But, the form of the Hamiltonian in eq. (3.20) suggests that the commutator is proportional to the generators of $\mathrm{GL}(L, \mathbb{R})$ because the only non-trivial commutator arises from

$$
\left[\left(\hat{\Phi}^{T} \hat{\Phi}\right)_{i j},\left(\hat{\Pi} \hat{\Pi}^{T}\right)^{l m}\right]=4 i \hat{\mathbf{G}}_{[i}^{[l} \delta_{j]}^{m]},
$$

where $\hat{\mathbf{G}}_{[i}^{[l} \delta_{j]}^{m]}=\frac{1}{4}\left(\hat{\mathbf{G}}_{i}^{l} \delta_{j}^{m}+\hat{\mathbf{G}}_{j}^{l} \delta_{i}^{m}+\hat{\mathbf{G}}_{i}^{m} \delta_{j}^{l}+\hat{\mathbf{G}}_{j}^{m} \delta_{i}^{l}\right)$. An explicit calculation shows that the commutator actually depends only on the $\operatorname{SL}(L, \mathbb{R})$ generator and the Hamiltonian itself (see appendix C),

$$
\begin{aligned}
{\left[\hat{H}_{u}, \hat{H}_{v}\right]=} & -i \frac{4 \tilde{\alpha}}{M^{2}} \operatorname{tr}\left\{\left[\left(\hat{\Pi} \hat{\Pi}^{T}\right) u\left(\hat{\Pi} \hat{\Pi}^{T}\right) v-\left(\hat{\Pi} \hat{\Pi}^{T}\right) v\left(\hat{\Pi} \hat{\Pi}^{T}\right) u\right] \hat{G}\right\} \\
& +i \frac{4 \tilde{\alpha}^{2}}{M^{4}} u_{n k} v_{n^{\prime} k^{\prime}}\left[-\left(\hat{\Pi} \hat{\Pi}^{T}\right)^{k l}\left(\hat{\Phi}^{T} \hat{\Phi}\right)_{l i}\left(\hat{\Pi} \hat{\Pi}^{T}\right)^{k^{\prime} i^{\prime}}\left(\hat{\Pi} \hat{\Pi}^{T}\right)^{j^{\prime} n^{\prime}} \delta_{j}^{n}\right. \\
& +\left(\hat{\Pi} \hat{\Pi}^{T}\right)^{k i^{\prime}}\left(\hat{\Phi}^{T} \hat{\Phi}\right)_{j l}\left(\hat{\Pi} \hat{\Pi}^{T}\right)^{l n^{\prime}}\left(\hat{\Pi} \hat{\Pi}^{T}\right)^{j^{\prime} n} \delta_{i}^{k^{\prime}} \\
& +\left(\hat{\Pi} \hat{\Pi}^{T}\right)^{k i^{\prime}}\left(\hat{\Pi} \hat{\Pi}^{T}\right)^{k^{\prime} l}\left(\hat{\Phi}^{T} \hat{\Phi}\right)_{l i}\left(\hat{\Pi} \hat{\Pi}^{T}\right)^{j^{\prime} n} \delta_{j}^{n^{\prime}} \\
& -\left(\hat{\Pi} \hat{\Pi}^{T}\right)^{k^{\prime} i^{\prime}}\left(\hat{\Pi} \hat{\Pi}^{T}\right)^{j^{\prime} n^{\prime}}\left(\hat{\Phi}^{T} \hat{\Phi}\right)_{j l}\left(\hat{\Pi} \hat{\Pi}^{T}\right)^{l n} \delta_{i}^{k} \\
& +M\left(\hat{\Pi} \hat{\Pi}^{T}\right)^{k i^{\prime}}\left(\hat{\Pi} \hat{\Pi}^{T}\right)^{j^{\prime} n^{\prime}} \delta_{i}^{k^{\prime}} \delta_{j}^{n}+(M+2)\left(\hat{\Pi} \hat{\Pi}^{T}\right)^{k i^{\prime}}\left(\hat{\Pi} \hat{\Pi}^{T}\right)^{k^{\prime} n} \delta_{i}^{j^{\prime}} \delta_{j}^{n^{\prime}} \\
& +2\left(\hat{\Pi} \hat{\Pi}^{T}\right)^{k i^{\prime}}\left(\hat{\Pi} \hat{\Pi}^{T}\right)^{j^{\prime} n} \delta_{i}^{k^{\prime}} \delta_{j}^{n^{\prime}}-2\left(\hat{\Pi} \hat{\Pi}^{T}\right)^{k n^{\prime}}\left(\hat{\Pi} \hat{\Pi}^{T}\right)^{k^{\prime} i^{\prime}} \delta_{i}^{j^{\prime}} \delta_{j}^{n} \\
& \left.-2\left(\hat{\Pi} \hat{\Pi}^{T}\right)^{k n^{\prime}}\left(\hat{\Pi} \hat{\Pi}^{T}\right)^{j^{\prime} i^{\prime}} \delta_{i}^{k^{\prime}} \delta_{j}^{n}-2\left(\hat{\Pi} \hat{\Pi}^{T}\right)^{n k}\left(\hat{\Pi} \hat{\Pi}^{T}\right)^{n^{\prime} i^{\prime}} \delta_{i}^{j^{\prime}} \delta_{j}^{k^{\prime}}\right] \hat{G}_{\left[i^{\prime}\right.}^{[i} j_{\left.j^{\prime}\right]}^{j]} \\
& +\frac{\tilde{\alpha}}{M^{2}}\left[(M-2) \operatorname{tr}\left\{\left(v \hat{\Pi} \hat{\Pi}^{T} u-u \hat{\Pi} \hat{\Pi}^{T} v\right) \hat{H}\right\}+4\left(\operatorname{tr}\left\{\hat{\Pi} \hat{\Pi}^{T} v\right\} \operatorname{tr}\{\hat{H} u\}\right.\right. \\
& \left.\left.-\operatorname{tr}\left\{\hat{\Pi} \hat{\Pi}^{T} u\right\} \operatorname{tr}\{\hat{H} v\}\right)\right],
\end{aligned}
$$

where $\hat{G}_{[i}^{l} \delta_{j]}^{m]}=\frac{1}{4}\left(\hat{G}_{i}^{l} \delta_{j}^{m}+\hat{G}_{j}^{l} \delta_{i}^{m}+\hat{G}_{i}^{m} \delta_{j}^{l}+\hat{G}_{j}^{m} \delta_{i}^{l}\right)$. It is noted that $\left(\hat{\Pi} \hat{\Pi}^{T}\right),\left(\hat{\Phi}^{T} \hat{\Phi}\right), \hat{G}, \hat{H} \sim$ $\mathrm{O}(M)$ in the large $M$ limit. The first two terms in eq. (3.27) are $\mathrm{O}(M)$. The last term that 
depends on $\hat{H}$ is $\mathrm{O}(1)$, and is sub-leading in the large $M$ limit. ${ }^{9}$ The last term is generated as operators are ordered such that $\hat{G}$ appears at the far right in the first two terms. This ordering makes it manifest that states annihilated by $\hat{G}$ and $\hat{H}$ are automatically annihilated by their commutators. Therefore, no additional constraints are needed to define the space of gauge invariant states. In short, the momentum and Hamiltonian constraints form a first class algebra [45]. We note that the first class algebra summarized in eqs. (3.24), (3.25) and (3.27) is the operator algebra that holds independent of states. For example, physical states that are annihilated by the constraints may or may not have the $\mathrm{O}(M)$ flavour symmetry.

\section{Path integral representation of state projection}

\subsection{Projection}

The momentum and Hamiltonian are generators of gauge transformations. The physical Hilbert space is given by the set of gauge invariant states. Let $|0\rangle$ be a gauge invariant state which satisfies

$$
\hat{G}_{y}|0\rangle=\hat{H}_{v}|0\rangle=0
$$

for any choice of $y, v$. An example of gauge invariant states is $\int D \Phi|\Phi\rangle$. However, this state has infinite norm with respect to the inner product defined in eq. (2.1). This is a property that all gauge invariant states share. Gauge invariant states are non-normalizable because the Hilbert space and the gauge group are both non-compact. Wavefunctions of gauge invariant states are necessarily extended over unbounded regions in the phase space as is proven in appendix B.

On the other hand, quantum states to which probabilities can be assigned should be normalizable. Wavefunctions of normalizable states are localized within compact regions in the gauge orbit, breaking gauge symmetry [25]. The fact that all normalizable states break the gauge symmetry has a few consequences. First, there exists no normalizable state that is frozen in time in this theory. All physical states, in the sense that they are normalizable, must evolve non-trivially in time. This provides one possible explanation for why we physically perceive the continuous passage of time although time evolution is merely a gauge transformation. Second, the emergent time should be non-compact. Perfect periodic orbits are impossible because the existence of a periodic gauge orbit of normalizable states implies a normalizable gauge invariant state.

The incompatibility between gauge invariance and normalizability gives rise to a nontrivial evolution as normalizable states are projected toward a gauge invariant state [46, 47]. Let us denote a normalizable state as $|\chi\rangle$. The projection of $|\chi\rangle$ to a gauge invariant state, $|0\rangle$ is given by

$$
\langle 0 \mid \chi\rangle
$$

\footnotetext{
${ }^{9} \operatorname{tr}\left\{\left[\left(v \hat{\Pi} \hat{\Pi}^{T} u-u \hat{\Pi} \hat{\Pi}^{T} v\right)\right] \hat{H}\right\}$ is only $\mathrm{O}(M)$ as the leading order term vanishes.
} 
This can be viewed as the wavefunction of $|0\rangle$ written in the basis of $|\chi\rangle[48,83]$. In the following, we show that eq. (4.2) can be written as a path integration of collective variables that describe fluctuations of emergent spactime.

\subsection{Gauge invariant local structure}

Under the generalized spatial diffeomorphism, the definition of local Hilbert spaces and the local structure associated with the local Hilbert spaces change. There is no preferred frame in priori. A gauge invariant local Hilbert spaces can only be defined with reference to physical degrees of freedom within the theory. This is possible within a sub-Hilbert space in which $\mathrm{O}(M)$ flavour symmetry is spontaneously broken down to a smaller group. We consider states in which the $\mathrm{O}(M)$ symmetry is broken as $\Phi$ acquires non-zero expectation values in an $L \times L$ block. If the $L \times L$ block of $\Phi$ is non-singular, $\mathrm{O}(M)$ symmetry is broken down to $\mathrm{O}(N)$, where $N=M-L$. We consider the sub-Hilbert space in which the $\mathrm{O}(N)$ flavour symmetry is further broken down to $\mathrm{O}(N / 2) \times \mathrm{O}(N / 2)$. In order to distinguish the first $L$, the next $N / 2$ and the remaining $N / 2$ flavours, we write

$$
\Phi_{i}^{A}=\left\{\begin{array}{cll}
\sqrt{N} q_{i}^{A} & \text { for } \quad A=1,2, \ldots, L \\
\phi_{i}^{A-L} & \text { for } \quad A=L+1, L+2, \ldots, L+N / 2 \\
\varphi_{i}^{A-(L+N / 2)} & \text { for } \quad A=L+N / 2+1, L+N / 2+2, \ldots, L+N
\end{array} .\right.
$$

$q$ represents an $L \times L$ matrix that acquire non-zero expectation values. A factor of $\sqrt{N}$ is introduced as we will consider the large $N$ limit with $\langle q\rangle \sim \mathrm{O}(1)$. Under generalized spatial diffeomorphisms, $q$ is transformed as $q \rightarrow q g$, where $g \in \operatorname{SL}(L, \mathbb{R})$, and plays the role of a Stueckelberg field. If $q$ contains $L$ independent row vectors, one can define a frame in terms of those vectors. The local structure defined in this frame is gauge invariant. $\phi$ and $\varphi$ represent $N / 2 \times L$ matrices that have zero expectation value. Henceforth, we use $|q, \phi, \varphi\rangle$ in place of $|\Phi\rangle$.

In the frame chosen by the Stueckelberg field, sites are labeled by distinguishable physical flavour. However, it is not necessary to have distinguishable sites to define a frame. It is sufficient to have $L$ unordered independent vectors. In order to define an unordered set of row vectors, we consider states in which $S_{L}^{f}$ symmetry is unbroken, where $S_{L}^{f}$ is the permutation that acts on the first $L$ flavours. We refer to $S_{L}^{f}$ as the flavour permutation group. We denote the sub-Hilbert space with unbroken $S_{L}^{f} \times \mathrm{O}(N / 2) \times \mathrm{O}(N / 2)$ as $\mathscr{V}$.

The choice of the sub-Hilbert space with this particular flavour group is not crucial. If one chooses different sub-Hilbert spaces, the collective modes that describe fluctuations within the sub-Hilbert spaces changes. The number of collective variables increases as the symmetry of the sub-Hilbert space is lowered. Here, we choose $S_{L}^{f} \times \mathrm{O}(N / 2) \times \mathrm{O}(N / 2)$ as an example that gives rise to a minimal set of physical degrees of freedom including dynamical gravity. The counting of the propagating degrees of freedom in this sub-Hilbert space is given at the end of section 4.4.

General states in $\mathscr{V}$ can be parameterized in terms of collective variables. In order to construct basis states for $\mathscr{V}$, it is convenient to introduce

$$
|q, \phi, \varphi\rangle^{\prime}=\sum_{P^{f} \in S_{L}^{f}}\left|P^{f} q, \phi, \varphi\right\rangle
$$


that are symmetric under permutations of the first $L$ flavours. While the expectation value of $\phi^{a}{ }_{i}$ and $\varphi^{a}{ }_{i}$ is zero, $\mathrm{O}(N / 2) \times \mathrm{O}(N / 2)$ invariant operators can still have nonzero expectation values. Any wavefunction in $\mathscr{V}$ written in the basis of eq. (4.4) can be expressed as a function of $q^{\alpha}{ }_{i},\left(\phi_{i}^{a} \phi^{a}{ }_{j}\right)$ and $\left(\varphi_{i}^{a} \varphi^{a}{ }_{j}\right)$, where the flavour $a$ is summed from 1 to $N / 2$ in the last two operators. Therefore, states in $\mathscr{V}$ can be spanned by the following basis states labeled by three collective variables,

$$
\left|s, t_{1}, t_{2}\right\rangle=\int D q D \phi D \varphi e^{i \operatorname{tr}\left\{N s q+t_{1}\left(\phi^{T} \phi\right)+t_{2}\left(\varphi^{T} \varphi\right)\right\}}|q, \phi, \varphi\rangle^{\prime},
$$

where $\operatorname{tr}\{s q\}=s^{i}{ }_{\alpha} q_{i}^{\alpha}, \quad \operatorname{tr}\left\{t_{1}\left(\phi^{T} \phi\right)\right\}=t_{1}^{i j} \phi^{a}{ }_{j} \phi_{i}^{a}, \quad \operatorname{tr}\left\{t_{2}\left(\varphi^{T} \varphi\right)\right\}=t_{2}^{i j} \varphi_{j}^{a}{ }_{j} \varphi_{i}^{a} . \quad s^{i}{ }_{\alpha}$ is the conjugate variable of $q_{i}^{\alpha}$. $t_{c}^{i j}$ with $c=1,2$ are bi-local variables that are conjugate to $\phi_{i}^{a} \phi^{a}{ }_{j}$ and $\varphi_{i}^{a} \varphi_{j}^{a}$, respectively. Both $t_{c}$ and $s$ are invariant under $\mathrm{O}(N / 2) \times \mathrm{O}(N / 2)$. Because $|q, \phi, \varphi\rangle^{\prime}$ in eq. (4.4) is invariant under flavour permutations, so is $\left|s, t_{1}, t_{2}\right\rangle$,

$$
\left|s, t_{1}, t_{2}\right\rangle=\left|s P^{f}, t_{1}, t_{2}\right\rangle
$$

for any $P_{f} \in S_{L}^{f}$. For a later use, we also introduce permutations of sites which act on the site index of the collective variables as

$$
s \rightarrow P_{g} s, \quad t_{c} \rightarrow P_{g} t_{c} P_{g}^{T} .
$$

The site permutation group with the even parity, denoted as $S_{L}^{g}$, is a subgroup of the generalized spatial diffeomorphism group, $\operatorname{SL}(L, \mathbb{R})$.

General states in $\mathscr{V}$ can be written as

$$
|\chi\rangle=\int D s D t\left|s, t_{1}, t_{2}\right\rangle \chi\left(s, t_{1}, t_{2}\right) \text {. }
$$

Here $D s \equiv \prod_{i, \alpha} d s^{i}{ }_{\alpha}, D t \equiv \prod_{i \geq j}\left[d t_{1}^{i j} d t_{2}^{i j}\right]$, and the integrations of $d s^{i}{ }_{\alpha}$ and $d t_{c}^{i j}$ are defined along the real axis. $\chi\left(s, t_{1}, t_{2}\right)$ is a wavefunction of the collective variables. The states in eq. (4.8) form a complete basis of $\mathscr{V}$. This sub-Hilbert space forms the kinematic Hilbert space of the present theory.

\subsection{Path integration of collective variables}

Now we consider the projection of a normalizable state in $\mathscr{V}$ to a gauge invariant state in eq. (4.2) in the limit that $N \gg L \gg 1$. Thanks to the gauge invariance of $|0\rangle$, the overlap in eq. (4.2) is invariant under gauge rotation [39],

$$
\langle 0 \mid \chi\rangle=\left\langle 0\left|e^{-i \epsilon\left(\hat{H}_{v^{(1)}}+\hat{G}_{y^{(1)}}\right)}\right| \chi\right\rangle,
$$

where $v^{(1)}$ is a lapse tensor and $y^{(1)}$ is a shift tensor. If $\hat{G}$ and $\hat{H}$ are applied to the right in eq. (4.9), they generate a non-trivial evolution of $|\chi\rangle$,

$$
\begin{aligned}
& e^{-i \epsilon\left(\hat{H}_{v^{(1)}}+\hat{G}_{y}(1)\right)}|\chi\rangle \\
& =\int D s^{(0)} D t^{(0)} \int D q D \phi D \varphi|q, \phi, \varphi\rangle^{\prime} e^{i \operatorname{tr}\left\{N s^{(0)} q+t_{1}^{(0)}\left(\phi^{T} \phi\right)+t_{2}^{(0)}\left(\varphi^{T} \varphi\right)\right\}} \times \\
& \quad e^{-i \epsilon N \operatorname{tr}\left\{v^{(1)} \mathscr{H}\left[q, s^{(0)}, \frac{\left(\phi^{T} \phi\right)}{N}, t_{1}^{(0)}, \frac{\left(\varphi^{T} \varphi\right)}{N}, t_{2}^{(0)}\right]+y^{(1)} \mathscr{G}\left[q, s^{(0)}, \frac{\left(\phi^{T} \phi\right)}{N}, t_{1}^{(0)}, \frac{\left(\varphi^{T} \varphi\right)}{N}, t_{2}^{(0)}\right]\right\}} \chi\left(s^{(0)}, t_{1}^{(0)}, t_{2}^{(0)}\right),
\end{aligned}
$$


where

$$
\begin{aligned}
\mathscr{H}\left[q, s, p_{1}, t_{1}, p_{2}, t_{2}\right]= & -\left(s s^{T}+\sum_{c}\left[4 t_{c} p_{c} t_{c}-i t_{c}\right]\right)+\tilde{\alpha}\left(s s^{T}+\sum_{c}\left[4 t_{c} p_{c} t_{c}-i t_{c}\right]\right) \\
& \times\left(q^{T} q+p_{1}+p_{2}\right)\left(s s^{T}+\sum_{c^{\prime}}\left[4 t_{c^{\prime}} p_{c^{\prime}} t_{c^{\prime}}-i t_{c^{\prime}}\right]\right)+O\left(\frac{1}{N}\right), \\
\mathscr{G}\left[q, s, p_{1}, t_{1}, p_{2}, t_{2}\right]= & \left(s q+2 \sum_{c} t_{c} p_{c}-i \frac{M}{2 N} I\right),
\end{aligned}
$$

and $s$ and $t_{c}$ in eq. (4.8) are relabeled as $s^{(0)}$ and $t_{c}^{(0)}$ in eq. (4.10). The evolution generated by the constraints is a manifestation of the fact that $|\chi\rangle$ is not gauge invariant. The resulting state in eq. (4.10) is also in $\mathscr{V}$, and can be written as a linear superposition of eq. (4.5). This is expressed as an integration over another set of collective variables and their conjugate variables $\left(q^{(1)}, s^{(1)}, p_{c}^{(1)}, t_{c}^{(1)}\right)$,

$$
\begin{aligned}
\langle 0 \mid \chi\rangle= & \int D s^{(0)} D t^{(0)} D s^{(1)} D t^{(1)} D q^{(1)} D p^{(1)}\langle 0| s^{(1)}, t_{1}^{(1)}, t_{2}^{(1)} \\
& \times e^{-i N \operatorname{tr}\left\{q^{(1)}\left(s^{(1)}-s^{(0)}\right)+p_{c}^{(1)}\left(t_{c}^{(1)}-t_{c}^{(0)}\right)\right\}} \\
& \times e^{-i \epsilon N \operatorname{tr}\left\{v^{(1)} \mathscr{H}\left[q^{(1)}, s^{(0)}, p_{1}^{(1)}, t_{1}^{(0)}, p_{2}^{(1)}, t_{2}^{(0)}\right]+y^{(1)} \mathscr{G}\left[q^{(1)}, s^{(0)}, p_{1}^{(1)}, t_{1}^{(0)}, p_{2}^{(1)}, t_{2}^{(0)}\right]\right\}} \chi\left(s^{(0)}, t_{1}^{(0)}, t_{2}^{(0)}\right) .
\end{aligned}
$$

It is straightforward to check that eq. (4.13) is reduced to eq. (4.10) upon integrating out the collective variables. Upon integrating over $s^{(1)}$ and $t_{c}^{(1)}$, which play the role of dynamical sources, one obtains the delta functions that enforce the constraints for the conjugate variables, $q^{(1)}=q, p_{1}^{(1)}=\frac{\phi^{T} \phi}{N}, p_{2}^{(1)}=\frac{\varphi^{T} \varphi}{N}$. The following integration over $q^{(1)}$ and $p_{c}^{(1)}$, which represent dynamical operators, reproduces eq. (4.10). The new set of dynamical collective variables in eq. (4.13) removes terms that are non-linear in $q^{\alpha}{ }_{i}$, $\phi_{i}^{a} \phi_{j}^{a}$ and $\varphi_{i}^{a} \varphi_{j}^{a}$ in eq. (4.10). Eq. (4.11) and eq. (4.12) represent the Hamiltonian and momentum constraints induced for the collective variables. Since eq. (4.10) is independent of the lapse and shift tensors, $v^{(1)}$ and $y^{(1)}$ can be integrated over in eq. (4.13). They can be viewed as Lagrangian multipliers that enforce the constraints.

We repeat the procedure by inserting $e^{-i \epsilon\left(\hat{H}_{v^{(2)}}+\hat{G}_{y^{(2)}}\right)}$ between $\langle 0|$ and $\left|s^{(1)}, t_{1}^{(1)}, t_{2}^{(1)}\right\rangle$ in eq. (4.13). This gives rise to an evolution of $\left|s^{(1)}, t_{1}^{(1)}, t_{2}^{(1)}\right\rangle$. The resulting state can be again expressed as a linear superposition of $\left|s, t_{1}, t_{2}\right\rangle$, which is expressed as an integration over a yet another set of dynamical collective variables. Repeated insertions of the Hamiltonian and momentum give rise to a path integration of the collective variables [49],

$$
\langle 0 \mid \chi\rangle=\int D s^{(0)} D t^{(0)} \int \mathscr{D} s \mathscr{D} t \mathscr{D} q \mathscr{D} p \mathscr{D} v \mathscr{D} y\left\langle 0 \mid s^{(\infty)}, t_{1}^{(\infty)}, t_{2}^{(\infty)}\right\rangle e^{i S} \chi\left(s^{(0)}, t_{1}^{(0)}, t_{2}^{(0)}\right),
$$

where

$$
\begin{aligned}
S= & N \int_{0}^{\infty} d \tau \operatorname{tr}\left\{-q \partial_{\tau} s-p_{c} \partial_{\tau} t_{c}-v(\tau) \mathscr{H}\left[q(\tau), s(\tau), p_{1}(\tau), t_{1}(\tau), p_{2}(\tau), t_{2}(\tau)\right]\right. \\
& \left.-y(\tau) \mathscr{G}\left[q(\tau), s(\tau), p_{1}(\tau), t_{1}(\tau), p_{2}(\tau), t_{2}(\tau)\right]\right\}
\end{aligned}
$$




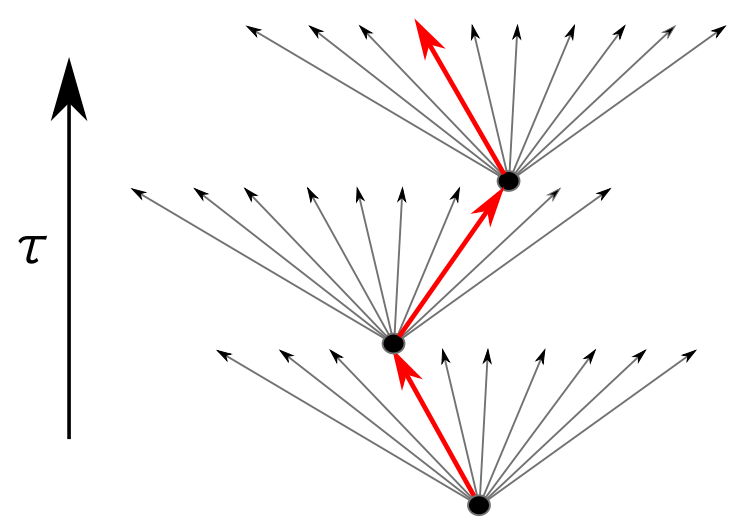

Figure 7. A state can be evolved by the constraints with different choices of lapse and shift tensors. A specific choice represents one of multi-fingered time evolutions.

Here $\mathscr{D} s \equiv \prod_{l=1}^{\infty} d s^{(l)}$ and $s(\tau)=s^{(l)}$ with $\tau=l \epsilon . \mathscr{D} q, \mathscr{D} t, \mathscr{D} p, \mathscr{D} v, \mathscr{D} y$, and $q(\tau), t_{c}(\tau)$, $p_{c}(\tau), v(\tau), y(\tau)$ are similarly defined. Here $\tau$ is a parameter time that labels different stages of evolution generated by the momentum and Hamiltonian constraints. Here $q$ and $p_{c}$ play the role of generalized coordinates and $s$ and $t_{c}$ are their conjugate momenta. ${ }^{10}$ The sum over the shift and lapse tensors in eq. (4.14) represents different paths in which the state in the kinematic Hilbert space is projected to the gauge invariant state. A particular path of the shift and lapse tensor represents one of the multi-fingered time evolutions. This is illustrated in figure 7. The collective variables $t_{c}(\tau), p_{c}(\tau), s(\tau), q(\tau)$ are generalized gravitational degrees of freedom that describe fluctuations of dimension, topology and geometry of spacetime. In the following section, we derive the precise relation between the collective variables and the metric of the emergent geometry.

\subsection{Gauge invariance of the action}

Because $\hat{H}$ and $\hat{G}$ obey the first-class constraint algebra, the action in eq. (4.15) is invariant under gauge trasformations generated by the constraints. To see this, we first note that the symplectic form in eq. (4.15) defines the Poisson bracket,

$$
\{A, B\}_{\mathrm{PB}}=\left(\frac{\partial A}{\partial q_{i}^{\alpha}} \frac{\partial B}{\partial s^{i}{ }_{\alpha}}-\frac{\partial A}{\partial s^{i}{ }_{\alpha}} \frac{\partial B}{\partial q_{i}^{\alpha}}\right)+\delta_{i j}^{k l}\left(\frac{\partial A}{\partial p_{c, i j}} \frac{\partial B}{\partial t_{c}^{k l}}-\frac{\partial A}{\partial t_{c}^{k l}} \frac{\partial B}{\partial p_{c, i j}}\right)
$$

where $\delta_{i j}^{k l}=\frac{1}{2}\left(\delta_{i}^{k} \delta_{j}^{l}+\delta_{i}^{l} \delta_{j}^{k}\right)$. To the leading order in $1 / N$, the Poisson brackets of eq. (4.11) and eq. (4.12) are given by (see appendix D)

$$
\begin{aligned}
\left\{\mathscr{G}^{i}, \mathscr{G}_{l}^{k}\right\}_{\mathrm{PB}} & =\mathscr{A}_{j l m}^{i k n} \mathscr{G}_{n}^{m}, \\
\left\{\mathscr{G}^{i}, \mathscr{H}^{k l}\right\}_{\mathrm{PB}} & =\mathscr{B}_{j m n}^{i k l} \mathscr{H}^{m n}, \\
\left\{\mathscr{H}^{i j}, \mathscr{H}^{k l}\right\}_{\mathrm{PB}} & =\mathscr{C}_{m}^{i j k l n} \mathscr{G}_{n}^{m},
\end{aligned}
$$

\footnotetext{
${ }^{10}$ An integration by part in eq. (4.15) gives the standard symplectic form, $\int d \tau \operatorname{tr}\left\{s \partial_{\tau} q+t_{c} \partial_{\tau} p_{c}\right\}$ with a boundary term.
} 
where

$$
\begin{aligned}
\mathscr{A}_{j l m}^{i k n}= & \delta_{j}^{k} \delta_{m}^{i} \delta_{l}^{n}-\delta_{l}^{i} \delta_{m}^{k} \delta_{j}^{n}, \\
\mathscr{B}_{j m n}^{i k l}= & \delta_{j}^{k} \delta_{m n}^{i l}+\delta_{j}^{l} \delta_{m n}^{k i}, \\
\mathscr{C}_{m}^{i j k l n}= & -4 \tilde{\alpha}\left[U^{n[j} U^{i][l} \delta_{m}^{k]}-U^{n[l} U^{k][j} \delta_{m}^{i]}\right] \\
& +4 \tilde{\alpha}^{2}\left[U^{n[j} U^{i] m^{\prime}} Q_{m^{\prime} n^{\prime}} U^{n^{\prime}[l} \delta_{m}^{k]}+U^{n[j} U^{i][l} U^{k] m^{\prime}} Q_{m^{\prime} n^{\prime}} \delta_{m}^{n^{\prime}}\right. \\
& \left.-U^{n[l} U^{k] m^{\prime}} Q_{m^{\prime} n^{\prime}} U^{n^{\prime}[j} \delta_{m}^{i]}-U^{n[l} U^{k][j} U^{i] m^{\prime}} Q_{m^{\prime} n^{\prime}} \delta_{m}^{n^{\prime}}\right]
\end{aligned}
$$

with

$$
\begin{aligned}
U^{i j} & =\left(s s^{T}+\sum_{c}\left[4 t_{c} p_{c} t_{c}-i t_{c}\right]\right)^{i j}, \\
Q_{i j} & =\left(q^{T} q+\sum_{c} p_{c}\right)_{i j} .
\end{aligned}
$$

In the last equation of eq. (4.20), the indices within brackets are symmetrized, e.g., $U^{n[j} U^{i][l} \delta_{m}^{k]}=\frac{1}{4}\left(U^{n j} U^{i l} \delta_{m}^{k}+U^{n i} U^{j l} \delta_{m}^{k}+U^{n j} U^{i k} \delta_{m}^{l}+U^{n i} U^{j k} \delta_{m}^{l}\right)$. Eqs. (4.17)-(4.19) have the same structure as eqs. (3.24), (3.25) and eq. (3.27) to the leading order in $1 / N$. The Hamiltonian that appears on the right hand side of eq. (3.27) is sub-leading.

The action in eq. (4.15) is invariant under the time-dependent gauge transformations,

$$
\begin{aligned}
\delta F & =\eta_{i}^{j}\left\{F, \mathscr{G}_{j}^{i}\right\}_{\mathrm{PB}}+\rho_{j i}\left\{F, \mathscr{H}^{i j}\right\}_{\mathrm{PB}}, \\
\delta v_{m n} & =\partial_{\tau} \rho_{m n}+\eta_{i}^{j} v_{l k} \mathscr{B}_{j m n}^{i k l}-\rho_{k l} y^{j}{ }_{i} \mathscr{B}_{j m n}^{i k l}, \\
\delta y_{m}^{n} & =\partial_{\tau} \eta_{m}^{n}+\eta_{i}^{j} y_{k}{ }_{k} \mathscr{A}_{j l m}^{i k n}+\rho_{j i} v_{l k} \mathscr{C}_{m}^{i j k l n},
\end{aligned}
$$

where $F=\{s, q, t, p\}$ denote the collective variables, and $\eta_{i}^{j}(\tau)$ and $\rho_{i j}(\tau)$ are infinitesimal gauge parameters. The action is invariant off-shell as the equation of motion is not needed for the invariance of the action. Besides the spacetime diffeomorphism, the theory is also invariant under the reversal of the parameter time,

$$
\begin{aligned}
i & \rightarrow-i, \\
\left\{p_{c}(\tau), q(\tau), v(\tau)\right\} & \rightarrow\left\{p_{c}(-\tau), q(-\tau), v(-\tau)\right\}, \\
\left\{t_{c}(\tau), s(\tau), y(\tau)\right\} & \rightarrow-\left\{t_{c}(-\tau), s(-\tau), y(-\tau)\right\} .
\end{aligned}
$$

Let us count the number of physical degrees of freedom. $q$ and $s$ are $L \times L$ matrices, and $t_{1}, t_{2}, p_{1}, p_{2}$ are $L \times L$ symmetric matrices. This give $D_{k}=2 L^{2}+2 L(L+1)$ phase space kinematic variables. On the other hand, $\mathscr{G}$ is traceless $L \times L$ matrix and $\mathscr{H}$ is $L \times L$ symmetric matrix. The total number of constraints is $D_{c}=\left(L^{2}-1\right)+\frac{L(L+1)}{2}$, and the dimension of the constraint hypersurface is $D_{k}-D_{c}$. The first-class constraints generate gauge orbits of dimension $D_{c}$ within the constraint hypersurface. Since points on a gauge orbit are physically identical, the total number of physical phase space variables is

$$
D_{k}-2 D_{c}=L(L+1)+2 .
$$




\section{Spacetime diffeomorphism and emergent geometry}

The path integral in eq. (4.14) consists of two parts. The first is the integration over $v(\tau)$ and $y(\tau)$. Each path of the lapse and shift tensors selects one of the multi-fingered time evolutions. Because of the gauge invariance in eq. (4.22), the shift and lapse tensors need to be fixed through a gauge fixing condition. This will be discussed in the next section. The remaining path integration is over the collective variables. Each path describes a history of the collective variables that represents a spacetime which emerges dynamically. In the large $N$ limit, the fluctuations of the collective variables become small, and the saddle-point approximation can be made. If the initial state $|\chi\rangle$ has a local structure in a frame, a spacetime manifold with well-defined dimension, topology and geometry emerges at the saddle point. In this section, we discuss how the geometry of the emergent manifold is determined from the collective variables. For this, we first extract the constraint algebra of the general relativity in eqs. (3.3)-(3.4) from eqs. (4.17)-(4.19).

\subsection{Momentum density}

We first identify the generators of smooth spacetime diffeomorphism for states that have local structures. Let $r_{i}^{\mu}$ be the mapping from sites to a manifold that is determined from the local structure in a frame. The tensor $\mathscr{G}_{j}{ }_{j}$ can be viewed as a bi-local field that depends on two positions on the manifold. If the collective variables change slowly in the manifold, $\mathscr{G}^{i}{ }_{j}$ varies slowly as a function of $r_{i}$ and $r_{j}$, and can be expanded in coordinates. By expanding $\mathscr{G}^{i}{ }_{j}$ around $j=i$, we write the $\mathrm{SL}(L, \mathbb{R})$ generator with shift tensor $y$ as

$$
\begin{aligned}
\mathscr{G}_{y} & =\mathscr{G}^{i}{ }_{j} y^{j}{ }_{i} . \\
& =\left[\mathscr{G}^{i}{ }_{i}+\left.\frac{\partial \mathscr{G}^{i}{ }_{j}}{\partial r_{j}^{\mu}}\right|_{j=i}\left(r_{j}^{\mu}-r_{i}^{\mu}\right)+\ldots\right] y^{j}{ }_{i} \\
& =\mathscr{G}_{i}^{i} \zeta_{y}\left(r_{i}\right)+\left.\frac{\partial \mathscr{G}^{i}{ }_{j}}{\partial r_{j}^{\mu}}\right|_{j=i} \xi_{y}^{\mu}\left(r_{i}\right)+\ldots,
\end{aligned}
$$

where $\zeta_{y}$ and $\xi_{y}^{\mu}$ represent the scalar and vector fields defined in eqs. (3.14)-(3.15) associated with shift tensor $y$. In the continuum limit, eq. (5.1) is written as

$$
\mathscr{G}_{y}=\int d r\left(\mathscr{D}(r) \zeta_{y}(r)+\mathscr{P}_{\mu}(r) \xi_{y}^{\mu}(r)+\ldots\right),
$$

where

$$
\begin{gathered}
\mathscr{D}\left(r_{i}\right)=V_{i}^{-1} \mathscr{G}_{i}^{i}, \\
\mathscr{P}_{\mu}\left(r_{i}\right)=\left.V_{i}^{-1} \frac{\partial \mathscr{G}^{i}{ }_{j}}{\partial r_{j}^{\mu}}\right|_{j=i} .
\end{gathered}
$$

$V_{i}$ is the coordinate volume assigned to site $i$ in the manifold (see figure 4). $V_{i}^{-1}$ in eq. (5.3) and eq. (5.4) densitizes the objects defined at sites. $\mathscr{D}\left(r_{i}\right)$ is the local dilatation density 
which generates the Weyl transformation. $\zeta_{y}$ corresponds to the temporal component of the non-compact Weyl U(1) gauge field in the Hamiltonian formalism. $\mathscr{P}_{\mu}\left(r_{i}\right)$ is identified as the momentum density, and $\xi_{y}$ becomes the shift. Now let us check that eq. (5.4) satisfies the constraint algebra of the general relativity given in eq. (3.3). The Poisson brackets of $\mathscr{D}\left(r_{i}\right)$ and $\mathscr{P}_{\mu}\left(r_{i}\right)$ are fixed by those of $\mathrm{SL}(L, \mathbb{R})$ generators. Eq. (4.17) implies $\left\{\mathscr{G}_{x}, \mathscr{G}_{y}\right\}_{\mathrm{PB}}=\mathscr{G}_{y x-x y}$, which can be written as

$$
\begin{aligned}
& \left\{\int d r\left(\mathscr{D}(r) \zeta_{x}(r)+\mathscr{P}_{\mu}(r) \xi_{x}^{\mu}(r)+\ldots\right), \int d r^{\prime}\left(\mathscr{D}\left(r^{\prime}\right) \zeta_{y}\left(r^{\prime}\right)+\mathscr{P}_{\nu}\left(r^{\prime}\right) \xi_{y}^{\nu}\left(r^{\prime}\right)+\ldots\right)\right\}_{\mathrm{PB}} \\
& =\int d r\left(\mathscr{D}(r) \zeta_{y x-x y}(r)+\mathscr{P}_{\mu}(r) \xi_{y x-x y}^{\mu}(r)+\ldots\right),
\end{aligned}
$$

where $\zeta_{y x-x y}(r)$ and $\xi_{y x-x y}^{\mu}(r)$ are the scalar and vector fields associated with the shift tensor, $y x-x y$. They are given by (see appendices E.1 and E.2 for derivation)

$$
\begin{aligned}
& \zeta_{y x-x y}(r)=\mathscr{L}_{\xi_{x}} \zeta_{y}(r)+O\left(\partial^{2}\right), \\
& \xi_{y x-x y}^{\mu}(r)=\left(\mathscr{L}_{\xi_{x}} \xi_{y}(r)\right)^{\mu}+O\left(\partial^{2}\right) .
\end{aligned}
$$

$O\left(\partial^{2}\right)$ denotes terms that involve two or more derivatives. Eqs. (5.6) and (5.7) imply that $\mathscr{P}_{\mu}$ defined in eq. (5.4) generates spatial diffeomorphism under which $\mathscr{D}$ and $\mathscr{P}_{\mu}$ transform as a scalar density and a vector density, respectively. Eq. (3.3) is indeed reproduced to the leading order in the derivative expansion.

\subsection{Hamiltonian density}

Now we write the Hamiltonian in the continuum limit. In a frame chosen by the local structure of a state, we divide the lapse tensor into the diagonal components and the off-diagonal components as

$$
\mathscr{H}_{v}=\sum_{i} \mathscr{H}^{i i} v_{i i}+\sum_{i \neq j} \mathscr{H}^{i j} v_{i j}
$$

In the continuum limit, eq. (5.8) can be written as

$$
\mathscr{H}_{v}=\int d r \theta_{v}(r) \mathscr{H}(r)+\int_{r^{\prime} \neq r} d r d r^{\prime} \lambda_{v}\left(r, r^{\prime}\right) \mathscr{H}^{(2)}\left(r, r^{\prime}\right) .
$$

Here,

$$
\begin{aligned}
\mathscr{H}\left(r_{i}\right) & =V_{i}^{-1} \mathscr{H}^{i i}, \\
\theta_{v}\left(r_{i}\right) & =v_{i i}
\end{aligned}
$$

are identified as the Hamiltonian density and the lapse function of the general relativity. The off-diagonal Hamiltonian and the off-diagonal lapse function are given by

$$
\begin{aligned}
\mathscr{H}^{(2)}\left(r_{i}, r_{j}\right) & =V_{i}^{-1} V_{j}^{-1} H^{i j}, \\
\lambda_{v}\left(r_{i}, r_{j}\right) & =v_{i j} .
\end{aligned}
$$


In the second term of eq. (5.9), the integration over $r^{\prime}$ excludes the region near $r^{\prime}=r$ with coordinate volume $V_{r}$. If one chooses the lapse that is diagonal in the frame, $\lambda_{v}\left(r_{i}, r_{j}\right)=0$. The off-diagonal contribution encodes the information about the mismatch between the frame chosen by the local structure and the frame in which the lapse tensor is diagonal.

While $\theta_{v}$ and $\lambda_{v}$ mix with each other under general $\operatorname{SL}(L, \mathbb{R})$ transformations, they don't mix to the leading order in the derivative expansion under smooth spatial diffeomorphism. Eq. (4.18) implies $\left\{\mathscr{G}_{x}, \mathscr{H}_{v}\right\}_{\mathrm{PB}}=\mathscr{H}_{v x+x^{T} v}$. This determines how the lapse function transforms under the spatial diffeomorphism in the continuum,

$$
\begin{aligned}
& \left\{\int d r^{\prime \prime}\left(\mathscr{D}\left(r^{\prime \prime}\right) \zeta_{x}\left(r^{\prime \prime}\right)+\mathscr{P}_{\mu}\left(r^{\prime \prime}\right) \xi_{x}^{\mu}\left(r^{\prime \prime}\right)+\ldots\right), \int d r \theta_{v}(r) \mathscr{H}(r)+\int_{r \neq r^{\prime}} d r d r^{\prime} \mathscr{H}^{(2)}\left(r, r^{\prime}\right) \lambda_{v}\left(r, r^{\prime}\right)\right\}_{\mathrm{PB}} \\
& =\int d r \theta_{v x+x^{T} v}(r) \mathscr{H}(r)+\int_{r \neq r^{\prime}} d r d r^{\prime} \mathscr{H}^{(2)}\left(r, r^{\prime}\right) \lambda_{v x+x^{T} v}\left(r, r^{\prime}\right),
\end{aligned}
$$

where the lapse function and the off-diagonal lapse function associated with $v x+x^{T} v$ are given by

$$
\begin{aligned}
\theta_{v x+x^{T} v}(r) & =2 \zeta_{x}(r) \theta_{v}(r)+\mathscr{L}_{\xi_{x}} \theta_{v}(r)+O\left(\partial^{2}\right), \\
\lambda_{v x+x^{T} v}\left(r, r^{\prime}\right) & =\left[\zeta_{x}(r)+\zeta_{x}\left(r^{\prime}\right)\right] \lambda_{v}\left(r, r^{\prime}\right)+\mathscr{L}_{\xi_{x}} \lambda_{v}\left(r, r^{\prime}\right)+\mathscr{L}_{\xi_{x}}^{\prime} \lambda_{v}\left(r, r^{\prime}\right)+O\left(\partial^{2}\right),
\end{aligned}
$$

where $\mathscr{L}_{\xi_{x}}$ and $\mathscr{L}_{\xi_{x}}^{\prime}$ represent the Lie derivative acting on $r$ and $r^{\prime}$, respectively. This is shown in appendix E.3. The Hamiltonian density in eq. (5.10) carries charge 2 under the Weyl transformation generated by $\mathscr{D}$, and transforms as a scalar density of weight 1 under the spatial diffeomorphism as expected. The off-diagonal Hamiltonian density in eq. (5.12) carries the same charge under the Weyl transformation, but transforms as a bi-local scalar density with weight 2 under the spatial diffeomorphism. The Hamiltonian density does not mix with the off-diagonal Hamiltonian density under the spatial diffeomorphism to the leading order in the derivative expansion. If $\lambda_{v}$ and $\zeta_{x}$ are turned off, eq. (3.4) is reproduced from eq. (5.15).

\subsection{Emergent metric}

In the general relativity, the Poisson bracket between two Hamiltonians is proportional to the momentum constraint with the structure factor given by the spatial metric and the signature of the metric as is shown in eq. (3.6). This implies that one can extract the spatial metric and the signature from the Poisson bracket of Hamiltonians. In the present theory, eq. (4.19) implies that the Poisson bracket between two Hamiltonians with different lapse tensors is proportional to $\mathscr{D}$ and $\mathscr{P}_{\mu}$ to the leading order in the derivative expansion in the present theory. This allows us to identify the spatial metric unambiguously in terms of the collective variables. Combining eqs. (4.19), (5.9) and (5.2), we obtain

$$
\begin{aligned}
& \left\{\int d r\left(\theta_{u}(r) \mathscr{H}(r)+\ldots\right), \int d r^{\prime}\left(\theta_{v}\left(r^{\prime}\right) \mathscr{H}\left(r^{\prime}\right)+\ldots\right)\right\}_{\mathrm{PB}} \\
& =\int d r\left(F^{\nu}(r) \mathscr{D}(r)+G^{\mu \nu}(r) \mathscr{P}_{\mu}(r)+\ldots\right)\left(\theta_{u}(r) \nabla_{\nu} \theta_{v}(r)-\theta_{v}(r) \nabla_{\nu} \theta_{u}(r)\right)+O\left(\partial^{2}\right),(5.1
\end{aligned}
$$


where

$$
\begin{aligned}
F^{\nu}\left(r_{m}\right) & =\frac{1}{2} \sum_{i, k, n} \mathscr{C}_{m}^{i i k k n}\left(r_{k}^{\nu}-r_{i}^{\nu}\right) \\
G^{\mu \nu}\left(r_{m}\right) & =\frac{1}{2} \sum_{i, k, n} \mathscr{C}_{m}^{i i k k n}\left(r_{n}^{\mu}-r_{m}^{\mu}\right)\left(r_{k}^{\nu}-r_{i}^{\nu}\right) .
\end{aligned}
$$

The derivation can be found in appendix E.4. The difference between two evolutions generated by Hamiltonians with different lapse tensors is given by an Weyl transformation and a spatial diffeomorphism. In order to identify the metric tensor, we decompose $G^{\mu \nu}$ into the symmetric and anti-symmetric parts as

$$
\begin{aligned}
-\mathcal{S} g^{\mu \nu} & =\frac{G^{\mu \nu}+G^{\nu \mu}}{2}, \\
b^{\mu \nu} & =\frac{G^{\mu \nu}-G^{\nu \mu}}{2} .
\end{aligned}
$$

The symmetric tensor is identified as $-\mathcal{S} g^{\mu \nu}$ in eq. (3.6). Here $g^{\mu \nu}$ is the spatial metric whose overall sign is chosen such that the signature of the first spatial component is positive. $\mathcal{S}$ is the signature of time relative that of the first spatial component. $b^{\mu \nu}$ is the antisymmetric component of $G^{\mu \nu}$.

In the generalized constraint algebra summarized in eqs. (5.5), (5.14) and (5.16), $\zeta_{x}$, $F^{\nu}, b^{\mu \nu}$ appear as extra dynamical fields besides the metric. The presence of such extra modes is expected because the bi-local collective variables can be viewed as an infinite tower of local fields with arbitrarily large spins once expanded on a manifold. The full theory in eq. (4.14) also includes non-perturbative modes associated with fluctuations of topology and dimension. In states in which the extra fields are turned off, the generalized constraint algebra in eqs. (5.5), (5.14) and (5.16) reduces to the hypersurface deformation algebra of the general relativity in eqs. (3.3), (3.4) and (3.5) up to the two derivative order in the gradient expansion.

\section{Classical equation of motion}

\subsection{Symmetry of semi-classical states}

We view the path integration in eq. (4.14) as the evolution of the initial state $|\chi\rangle$ in eq. (4.8) under the change of parameter time $\tau$. Since $N$ plays the role of the inverse of the Planck constant in eq. (4.15), the path integration in eq. (4.14) can be approximated by the saddle-point solution in the large $N$ limit, provided that the initial state is chosen to be a semi-classical state. The semi-classical wavefunction for the collective variable can be written as

$$
\begin{aligned}
& \chi_{q, s, p_{c}, t_{c}}\left(s, t_{1}, t_{2}\right)= \\
& \exp \left(-i N \operatorname{tr}\left\{q s+\sum_{c} p_{c} t_{c}\right\}-\frac{\sum_{i, \alpha}\left[(s)^{i}{ }_{\alpha}-s^{i}{ }_{\alpha}\right]^{2}+\sum_{c} \sum_{i j}\left[t_{c}^{i j}-t_{c}^{i j}\right]^{2}}{\Delta^{2}}\right) .
\end{aligned}
$$


In the $\frac{1}{N} \ll \Delta \ll 1$ limit, the wave packet has well-defined collective variables and conjugate momenta peaked at $s=s, t_{c}=t_{c}, q=q, p_{c}=p_{c}$. While the semi-classical states are labeled by $\left\{s, t_{c}, q, p_{c}\right\}$, there is a redundancy in labeling states in terms of the classical variables. Because the basis state in eq. (4.6) is chosen to be invariant under the permutations that exchange the first $L$ flavours, wavefunctions obtained by applying the flavour permutations to $q$, s represent the same physical state,

$$
\chi_{P_{f} q, s P_{f}^{T}, p_{c}, t_{c}}\left(s, t_{1}, t_{2}\right) \sim \chi_{q, s, p_{c}, t_{c}}\left(s, t_{1}, t_{2}\right),
$$

where $P_{f}^{T}$ is the transpose of $P_{f}$.

The symmetry of the semi-classical state is determined from the symmetry of the classical variables. If $q$ contains $L$ linearly independent row vectors, the vectors can be used as a frame. Since the frame can not be invariant under any infinitesimal $\operatorname{SL}(L, \mathbb{R})$ transformation, there is no unbroken continuous $\mathrm{SL}(L, \mathbb{R})$ symmetry. However, a discrete subgroup of $\mathrm{SL}(L, \mathbb{R})$ can be still preserved. Under the even site permutation group, which is a discrete subgroup of $\operatorname{SL}(L, \mathbb{R})$, the wavefunction is transformed as

$$
\chi_{q, s, p_{c}, t_{c}}\left(s, t_{1}, t_{2}\right) \rightarrow \chi_{q, s, p_{c}, t_{c}}\left(P_{g} s, P_{g} t_{1} P_{g}^{T}, P_{g} t_{2} P_{g}^{T}\right)
$$

as is shown in eq. (4.7). The right hand side of eq. (6.3) can be written as

$$
\chi_{q, s, \not_{c}, t_{c}}\left(P_{g} s, P_{g} t_{1} P_{g}^{T}, P_{g} t_{2} P_{g}^{T}\right)=\chi_{q} P_{g}, P_{g}^{T s}, P_{g}^{T} p_{c} P_{g}, P_{g}^{T} t_{c} P_{g}\left(s, t_{1}, t_{2}\right)
$$

because eq. (6.1) satisfies $\chi_{q} P^{T}, P s, P p_{c} P^{T}, P t_{c} P^{T}\left(P s, P t_{1} P^{T}, P t_{2} P^{T}\right)=\chi_{q, s, p_{c}, t_{c}}\left(s, t_{1}, t_{2}\right)$ for any site permutation, $P$. In eq. (6.4), $P^{T}=P^{-1}$ is used. Because of the redundancy in labeling physical states in terms of the collective variables, the state is invariant under eq. (6.3) if there exists a flavour permutation, $P_{f}$ that satisfies

$$
\begin{aligned}
q P_{g} & =P_{f} q, \quad P_{g}^{T} s=s P_{f}^{T}, \\
P_{g}^{T} p_{c} P_{g} & =p_{c}, \quad P_{g}^{T} t_{c} P_{g}=t_{c} .
\end{aligned}
$$

If there exists such $P_{f}$, the site permutation can be canceled by a flavour permutation through eq. (6.2). In this case, eq. (6.3) is a symmetry of the state,

$$
\chi_{q, s, p_{c}, t_{c}}\left(P_{g} s, P_{g} t_{1} P_{g}^{T}, P_{g} t_{2} P_{g}^{T}\right) \sim \chi_{q, s, p_{c}, t_{c}}\left(s, t_{1}, t_{2}\right) .
$$

Therefore, the unbroken subgroup of $\mathrm{SL}(L, \mathbb{R})$ is given by the set of $P_{g}$ that satisfies eq. (6.5) for some $P_{f}$. This subgroup is denoted as $\mathscr{I}_{q, s, p_{c}, t_{c}}$.

Let us examine the symmetry of the classical state in which $q$ is non-degenerate. In this case, it is convenient to choose the gauge in which $q$ is proportional to the identity matrix. With a generic choice of the remaining variables, $s, p_{c}, t_{c}, \mathrm{SL}(L, \mathbb{R})$ is completely broken, and $\mathscr{I}_{q, s, p_{c}, t_{c}}=\emptyset$. The maximal subset of $\operatorname{SL}(L, \mathbb{R})$ can be preserved if $s, p_{c}$, $t_{c}$ are all proportional to the identity matrix. This corresponds to a direct product state because all collective variables are diagonal. In this case, the full site permutation group remains unbroken: $\mathscr{I}_{q, s, p_{c}, t_{c}}=S_{L}^{g}$. This is because $P_{f}=P_{g}$ satisfies eq. (6.5) for any 


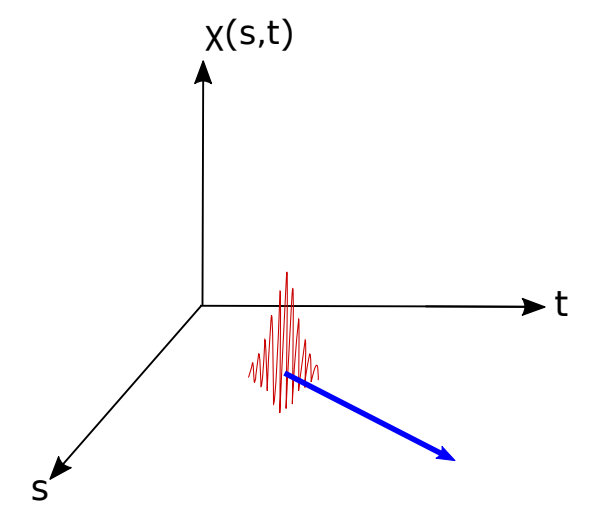

Figure 8. A wave packet that has a well-defined collective variables and the conjugate momenta. Under the time evolution, the classical variables evolve obeying the saddle-point equation.

$P_{g} \in S_{L}^{g}$. In order to have a non-trivial classical manifold, one needs to turn on off-diagonal elements of the classical variables that generate inter-site entanglement. If $(s q), p_{c}$ and $t_{c}$ have non-zero off-diagonal elements but $(s q)^{i}{ }_{j},\left(p_{c}\right)_{i j},\left(t_{c}\right)^{i j}$ depend only on $r_{i}-r_{j}$ in a manifold associated with a local structure, the state has an unbroken discrete translational symmetry. This discrete space symmetry can be enhanced to the continuous translation in the long distance limit. In section 7 , it will be shown that a discrete translational and rotational symmetry can be enhanced to a continuous group at long distances. We will also discuss an example in which the full Lorentz symmetry emerges as an isometry of the spacetime.

\subsection{Saddle-point equation of motion}

In the large $N$ limit, the path integration is dominated by the semi-classical path that satisfies the saddle-point equation of motion,

$$
\begin{aligned}
\partial_{\tau} \bar{t}_{c}= & 4 \bar{t}_{c} v \bar{t}_{c}-4 \tilde{\alpha}\left(\bar{t}_{c} \bar{Q} \bar{U} v \bar{t}_{c}+\bar{t}_{c} v \bar{U} \bar{Q} \bar{t}_{c}\right)-\tilde{\alpha} \bar{U} v \bar{U}-y \bar{t}_{c}-\bar{t}_{c} y^{T}, \\
\partial_{\tau} \bar{p}_{c}= & -\left[4 \bar{p}_{c} \bar{t}_{c} v+4 v \bar{t}_{c} \bar{p}_{c}-i v-4 \tilde{\alpha}\left(\bar{p}_{c} \bar{t}_{c} \bar{Q} \bar{U} v+v \bar{U} \bar{Q}_{c} \bar{p}_{c}\right)\right. \\
& \left.-4 \tilde{\alpha}\left(\bar{Q} \bar{U} v \bar{t}_{c} \bar{p}_{c}+\bar{p}_{c} \bar{t}_{c} v \bar{U} \bar{Q}\right)+i \tilde{\alpha}(\bar{Q} \bar{U} v+v \bar{U} \bar{Q})\right]+\bar{p}_{c} y+y^{T} \bar{p}_{c} \\
\partial_{\tau} \bar{s}= & -2 \tilde{\alpha} \bar{U} v \bar{U} \bar{q}^{T}-y \bar{s} \\
\partial_{\tau} \bar{q}= & -2 \bar{s}^{T} v+2 \tilde{\alpha}\left(\bar{s}^{T} \bar{Q} \bar{U} v+\bar{s}^{T} v \bar{U} \bar{Q}\right)+\bar{q} y
\end{aligned}
$$

with the initial condition, $\bar{t}_{c}(0)=t_{c}, \bar{p}_{c}(0)=p_{c}, \bar{s}(0)=s$ and $\bar{q}(0)=q \cdot y(\tau)$ and $v(\tau)$ are the time dependent shift and lapse tensors, respectively. They are free parameters. $y(\tau)$ sets the generalized spatial diffeomorphism within the constant time slice at $\tau$. The lapse tensor at each $\tau$ determines the rate of time evolution and the frame in which time evolution is generated.

In the large $N$ limit, eq. (6.1) can have a non-zero overlap with a gauge invariant state in eq. (4.2) only if the collective variables satisfy the constraints classically. This implies that one can not choose the classical values of the collective variables and their conjugate 
momenta arbitrarily. The classical collective variables should satisfy

$$
\begin{aligned}
\operatorname{tr}\left\{\left(\bar{s} \bar{q}+2 \sum_{c} \bar{t}_{c} \bar{p}_{c}-\frac{i}{2} I\right) y\right\} & =0, \\
\operatorname{tr}\{(-\bar{U}+\tilde{\alpha} \bar{U} \bar{Q} \bar{U}) v\} & =0
\end{aligned}
$$

for any traceless tensor $y$ and symmetric tensor $v$ at all $\tau$. In the phase space of the collective variables, the classical constraint hypersurface is defined by

$$
\begin{aligned}
\bar{s} \bar{q}+2 \sum_{c} \bar{t}_{c} \bar{p}_{c}-\frac{i}{2} I & =\beta I, \\
\tilde{\alpha} \bar{Q} \bar{U} & =I,
\end{aligned}
$$

where $\beta$ is an arbitrary constant. Thanks to the first-class nature of the constraints, eqs. (6.8) and (6.9) are conserved under the time evolution within the constraint hypersurface. Furthermore, $\beta$ is a constant of motion on the constraint hypersurface: the equations of motion in eq. (6.7) leads to

$$
\begin{aligned}
& \frac{\partial}{\partial \tau}\left(\bar{s} \bar{q}+2 \sum_{c} \bar{t}_{c} \bar{p}_{c}-\frac{i}{2} I\right)= \\
& 2(-\bar{U}+\tilde{\alpha} \bar{U} \bar{Q} \bar{U}) v-y\left(\bar{s} \bar{q}+2 \sum_{c} \bar{t}_{c} \bar{p}_{c}\right)+\left(\bar{s} \bar{q}+2 \sum_{c} \bar{t}_{c} \bar{p}_{c}\right) y .
\end{aligned}
$$

This vanishes on the constraint hypersurface for any value of $\beta$.

A few remarks are in order regarding the constraints that classical variables satisfy. First, the fact that the initial classical data should satisfy the constraints is a common feature of constraint systems including the general relativity. In the present theory, this is imposed through the condition that the state in the kinematic Hilbert space has a nonzero projection with a gauge invariant state in the large $N$ limit. Second, this is closely related to the well-known source-operator relation in the AdS/CFT correspondence $[9,10,50]$. In the AdS/CFT correspondence, sources fixed at a UV boundary (say through the Dirichlet boundary condition) fix their conjugate variables to avoid singularity in the bulk. In the present case, the collective variables (sources) and their conjugate momenta (operators) should satisfy the constraints to make sure that the overlap between the late time state given by

$$
|\chi(\tau)\rangle=\mathscr{T} e^{-i \int_{0}^{\tau} d \tau^{\prime}\left[\hat{H}_{v\left(\tau^{\prime}\right)}+\hat{G}_{y\left(\tau^{\prime}\right)}\right]}|\chi\rangle,
$$

and the gauge invariant state is non-zero in the large $N$ limit, where $\mathscr{T}$ time-orders the operators. If the collective variables do not satisfy the constraints classically, the state in eq. (6.13) will have a zero overlap with $\langle 0|$ in eq. (4.14) in the large $N$ limit. This is because the initial state and the final state have different 'energies' and 'momenta', and the two states are orthogonal to each other in the large $N$ limit. In this case, there is no classical path that connects the initial state to the final state. Alternatively, the vanishing overlap 
between the state at large $\tau$ and the gauge invariant state can be translated into a divergent boundary action present at future infinity in the path integration. A smooth boundary condition that avoids the divergent action (a catastrophic collapse of the wavefunction) at future infinity can be imposed only if the initial state satisfies the constraints classically. Third, the fact that the classical variables satisfy the constraints guarantees that $|\chi\rangle$ is invariant under the gauge transformation to the leading order in the large $N$ limit. The gauge symmetry is broken at the sub-leading order. This sub-leading gauge symmetry breaking is what makes sure that the state is normalizable and evolves non-trivially under time evolution [25].

\subsection{Gauge fixing}

The shift and lapse tensors are arbitrary, and we can choose them through a gauge fixing. It is convenient to use some physical degrees of freedom as a set of local clocks that fixes a frame in which a gauge invariant local structure is defined and time evolution is generated. To be concrete, let us consider an initial state with $\operatorname{det} \bar{q}(0)>0$. Without loss of generality, we can choose our initial frame such that $\bar{q}(0)$ is proportional to the identity matrix. Furthermore, we can make sure that $\bar{q}(\tau)$ remains proportional to the identity matrix at all $\tau$ by choosing the shift as

$$
y=\frac{1}{\bar{q}}(\bar{W}-<\bar{W}>I)
$$

where

$$
\bar{W}=2 \bar{s}^{T} v-2 \tilde{\alpha}\left(\bar{s}^{T} \bar{Q} \bar{U} v+\bar{s}^{T} v \bar{U} \bar{Q}\right)
$$

and $\left\langle\bar{W}>=\frac{\operatorname{tr}\{\bar{W}\}}{L}\right.$. In this gauge, $\bar{q}(\tau)$ remains proportional to the identity matrix at all $\tau$, and $\bar{q}(\tau)$ can be written as

$$
\bar{q}(\tau)=\bar{q}_{d}(\tau) I
$$

where $q_{d}$ is a single variable that represents the diagonal elements. This amounts to choosing a frame at each moment of time in terms of the $L$ independent vectors of $\bar{q}$. This frame defines a gauge invariant set of local Hilbert spaces and a local structure associated with them. The gauge freedom associated with the lapse tensor can be fixed so that the lapse tensor is diagonal in the frame in which $q$ is diagonal. This describes the time evolution of the system relative to a set of local clocks defined in the frame chosen by $q$. Here, we choose a uniform lapse tensor as

$$
v=I \text {. }
$$


We call eq. (6.14) and eq. (6.17) the unitary gauge condition. In the unitary gauge, the equations of motion become

$$
\begin{aligned}
\partial_{\tau} \bar{t}_{c}= & 4 \bar{t}_{c}^{2}-4 \tilde{\alpha}\left(\bar{t}_{c} \bar{Q} \bar{U} \bar{t}_{c}+\bar{t}_{c} \bar{U} \bar{Q}_{c}\right)-\tilde{\alpha} \bar{U}^{2}-\frac{1}{\bar{q}_{d}}\left(\bar{W} \bar{t}_{c}+\bar{t}_{c} \bar{W}^{T}-2<\bar{W}>\bar{t}_{c}\right), \\
\partial_{\tau} \bar{p}_{c}= & -\left[4 \bar{p}_{c} \bar{t}_{c}+4 \bar{t}_{c} \bar{p}_{c}-i-4 \tilde{\alpha}\left(\bar{p}_{c} \bar{t}_{c} \bar{Q} \bar{U}+\bar{U} \bar{Q} \bar{t}_{c} \bar{p}_{c}\right)-4 \tilde{\alpha}\left(\bar{Q} \bar{U} \bar{t}_{c} \bar{p}_{c}+\bar{p}_{c} \bar{t}_{c} \bar{U} \bar{Q}\right)\right. \\
& +i \tilde{\alpha}(\bar{Q} \bar{U}+\bar{U} \bar{Q})]+\frac{1}{\bar{q}_{d}}\left(\bar{p}_{c} \bar{W}+\bar{W}^{T} \bar{p}_{c}-2<\bar{W}>\bar{p}_{c}\right), \\
\partial_{\tau} \bar{s}= & -2 \tilde{\alpha} \bar{q}_{d} \bar{U}^{2}-\frac{1}{\bar{q}_{d}}(\bar{W}-<\bar{W}>) \bar{s} \\
\partial_{\tau} \bar{q}_{d}= & -<\bar{W}>.
\end{aligned}
$$

The equation of motion is simplified in terms of a new variable,

$$
\tilde{t}_{c}=\bar{t}_{c}-\frac{i}{8} \bar{p}_{c}^{-1} .
$$

On the constraint hypersurface, $W=-2 s^{T}$ in eq. (6.15), and the equation of motion for $\tilde{t}_{c}$ becomes

$$
\begin{aligned}
\partial_{\tau} \tilde{t}_{c} & =-4 \tilde{t}_{c}^{2}+\frac{1}{16} \bar{p}_{c}^{-2}-\tilde{\alpha} \bar{U}^{2}+\frac{2}{\bar{q}_{d}}\left(\bar{s}^{T} \tilde{t}_{c}+\tilde{t}_{c} \bar{s}-2<\bar{s}>\tilde{t}_{c}\right), \\
\partial_{\tau} \bar{p}_{c} & =4 \bar{p}_{c} \tilde{t}_{c}+4 \tilde{t}_{c} \bar{p}_{c}-\frac{2}{\bar{q}_{d}}\left(\bar{p}_{c} \bar{s}^{T}+\bar{s} \bar{p}_{c}-2<\bar{s}>\bar{p}_{c}\right), \\
\partial_{\tau} \bar{s} & =-2 \tilde{\alpha} \bar{q}_{d} \bar{U}^{2}+\frac{2}{\bar{q}_{d}}\left(\bar{s}^{T}-<\bar{s}>\right) \bar{s}, \\
\partial_{\tau} \bar{q}_{d} & =2<\bar{s}>,
\end{aligned}
$$

where $\left\langle\bar{s}>\equiv \frac{\operatorname{tr}\{\bar{s}\}}{L}, \bar{U}=\bar{s} \bar{s}^{T}+\sum_{c}\left[4 \tilde{t}_{c} \bar{p}_{c} \tilde{t}_{c}+\frac{1}{16} \bar{p}_{c}^{-1}\right]\right.$.

\section{Translationally invariant solution}

In this section, we solve eqs. (6.23)-(6.26) for an initial state that exhibits a $D$-dimensional local structure with a translational invariance. We choose a state with

$$
\begin{aligned}
\tilde{t}_{1}, \tilde{t}_{2} & =\tilde{t}, \\
\bar{p}_{1}, \bar{p}_{2} & =\frac{\bar{p}}{2},
\end{aligned}
$$

where $\tilde{t}$ and $\bar{p}$ describe a $D$-dimensional manifold with topology, $T^{D}$. For those states, it is natural to label sites in terms of $D$ integers that form the $D$-dimensional hyper-cubic lattice,

$$
\mathbf{r}=\left(n_{1}, n_{2}, \ldots, n_{D}\right)
$$


for $1 \leq n_{i} \leq L^{1 / D}$ with the identification $n_{i} \sim n_{i}+L^{1 / D}$. If the state has the $D$-dimensional translational invariance, collective variables can be represented in the momentum space,

$$
\begin{aligned}
& \tilde{t}_{\mathbf{k}}=\sum_{\mathbf{r}} e^{-i \mathbf{k r} r} \tilde{t}^{\tilde{r}^{\prime}+\mathbf{r}, \mathbf{r}^{\prime}}, \\
& \bar{p}_{\mathbf{k}}=\sum_{\mathbf{r}} e^{-i \mathbf{k r}} \bar{p}_{\mathbf{r}^{\prime}+\mathbf{r}, \mathbf{r}^{\prime}}, \\
& \bar{s}_{\mathbf{k}}=\sum_{\mathbf{r}} e^{-i \mathbf{k r}} \bar{s}_{\mathbf{r}^{\prime}+\mathbf{r}}^{\mathbf{r}^{\prime}},
\end{aligned}
$$

where $\mathbf{k}=\frac{2 \pi}{L^{1 / D}}\left(l_{1}, l_{2}, \ldots, l_{D}\right)$ with $-\frac{L^{1 / D}}{2} \leq l_{i}<\frac{L^{1 / D}}{2}$. For simplicity, we consider the case with the reflection symmetry and the discrete rotational symmetry. This guarantees $\tilde{t}_{\mathbf{k}}=\tilde{t}_{\mathbf{k}^{\prime}}, \bar{p}_{\mathbf{k}}=\bar{p}_{\mathbf{k}^{\prime}}, \bar{s}_{\mathbf{k}}=\bar{s}_{\mathbf{k}^{\prime}}$, and $\tilde{t}_{\mathbf{k}}=\tilde{t}_{\mathbf{R k}}, \bar{p}_{\mathbf{k}}=\bar{p}_{\mathbf{R k}}, \bar{s}_{\mathbf{k}}=\bar{s}_{\mathbf{R k}}$. Here $\mathbf{k}^{\prime}=\left(k_{1}, \ldots, k_{l-1},-k_{l}, k_{l+1}, \ldots, k_{D}\right)$ for some $l$. $\mathbf{R}$ is a $\frac{\pi}{2}$-rotation on any of the principal planes in the $D$-dimensional hypercubic lattice.

The hyper-cubic lattice breaks the continuous rotational symmetry to the discrete group. This is a spontaneously broken symmetry that is determined from the pattern of entanglement in the state. Nonetheless, we expect that the continuous rotational symmetry emerges at long distance scales. To see this, one can expand the collective variables in powers of $\mathbf{k}$,

$$
\tilde{t}_{\mathbf{k}}=\sum_{n} \tilde{t}^{\mu_{1} \mu_{2} \ldots \mu_{n}} k_{\mu_{1}} k_{\mu_{2}} \ldots k_{\mu_{n}}
$$

where $t^{\mu_{1} \mu_{2} \ldots \mu_{n}}$ represents a field with spin $n$. Due to the reflection symmetry, all odd spin fields vanish. Furthermore, the discrete rotational symmetry guarantees that $t^{\mu \nu} \propto \delta^{\mu \nu}$, and any spin 2 field should respect the full rotational symmetry. This implies that the spatial metric should be invariant under the full rotational symmetry as well. Higher spin fields do break the continuous rotational symmetry, but they become less important in the small k limit.

The collective variables at each $\mathbf{k}$ satisfy

$$
\begin{aligned}
& \partial_{\tau} \tilde{t}_{\mathbf{k}}=-4 \tilde{t}_{\mathbf{k}}^{2}+\frac{1}{4} \bar{p}_{\mathbf{k}}^{-2}-\tilde{\alpha} \bar{U}_{\mathbf{k}}^{2}+\frac{2}{\bar{q}_{d}}\left(\bar{s}_{-\mathbf{k}} \tilde{t}_{\mathbf{k}}+\tilde{t}_{\mathbf{k}} \bar{s}_{\mathbf{k}}-2<\bar{s}>\tilde{t}_{\mathbf{k}}\right), \\
& \partial_{\tau} \bar{p}_{\mathbf{k}}=8 \bar{p}_{\mathbf{k}} \tilde{t}_{\mathbf{k}}-\frac{2}{\bar{q}_{d}}\left(\bar{p}_{\mathbf{k}} \bar{s}_{-\mathbf{k}}+\bar{s}_{\mathbf{k}} \bar{p}_{\mathbf{k}}-2<\bar{s}>\bar{p}_{\mathbf{k}}\right), \\
& \partial_{\tau} \bar{s}_{\mathbf{k}}=-2 \tilde{\alpha} \bar{q}_{d} \bar{U}_{\mathbf{k}}^{2}+\frac{2}{\bar{q}_{d}}\left(\bar{s}_{-\mathbf{k}}-<\bar{s}>\right) \bar{s}_{\mathbf{k}}, \\
& \partial_{\tau} \bar{q}_{d}=2<\bar{s}>
\end{aligned}
$$

where

$$
\bar{U}_{\mathbf{k}}=\bar{s}_{\mathbf{k}} \bar{s}_{-\mathbf{k}}+4 \tilde{t}_{\mathbf{k}}^{2} \bar{p}_{\mathbf{k}}+\frac{1}{4} \bar{p}_{\mathbf{k}}^{-1}
$$

$<\bar{s}>=\frac{1}{L} \sum_{\mathbf{k}} \bar{s}_{\mathbf{k}}$, and we use $\left(\bar{p}^{-1}\right)_{\mathbf{k}}=\left(\bar{p}_{\mathbf{k}}\right)^{-1}$. 
The constraints in eqs. (6.10) and (6.11) imply

$$
\begin{array}{r}
\bar{q}_{d} \bar{s}_{\mathbf{k}}+2 \tilde{t}_{\mathbf{k}} \bar{p}_{\mathbf{k}}=\beta, \\
\tilde{\alpha}\left(\bar{q}_{d}^{2}+\bar{p}_{\mathbf{k}}\right)\left(\bar{s}_{\mathbf{k}} \bar{s}_{-\mathbf{k}}+4 \tilde{t}_{\mathbf{k}}^{2} \bar{p}_{\mathbf{k}}+\frac{1}{4} \bar{p}_{\mathbf{k}}^{-1}\right)=1
\end{array}
$$

for all $\mathbf{k}$. We can use the constraints to solve $s_{\mathbf{k}}$ and $\tilde{t}_{\mathbf{k}}$ in terms of $\bar{p}_{\mathbf{k}}$ and $\bar{q}_{d}$ as

$$
\begin{aligned}
& s_{\mathbf{k}}=\frac{1}{\bar{q}_{d}}\left(\beta-2 \tilde{t}_{\mathbf{k}} \bar{p}_{\mathbf{k}}\right), \\
& \tilde{t}_{\mathbf{k}}=T_{ \pm}\left[\bar{p}_{\mathbf{k}}, \bar{q}_{d}\right],
\end{aligned}
$$

where

$$
T_{ \pm}\left[\bar{p}_{\mathbf{k}}, \bar{q}_{d}\right]=\frac{2 \beta \bar{p}_{\mathbf{k}} \pm \bar{q}_{d} \sqrt{\gamma^{2} \bar{p}_{\mathbf{k}}-\bar{q}_{d}^{2}}}{4 \bar{p}_{\mathbf{k}}\left(\bar{p}_{\mathbf{k}}+\bar{q}_{d}^{2}\right)}
$$

with $\gamma \equiv \sqrt{\frac{1}{\tilde{\alpha}}\left(4-\tilde{\alpha}-4 \tilde{\alpha} \beta^{2}\right)}$. Here we consider $\tilde{\alpha}>0$ and $\beta>0$ with $\frac{1}{\tilde{\alpha}}\left(4-\tilde{\alpha}-4 \tilde{\alpha} \beta^{2}\right)>$ 0 . Here $T_{+}\left[\bar{p}_{\mathbf{k}}, \bar{q}_{d}\right]$ and $T_{-}\left[\bar{p}_{\mathbf{k}}, \bar{q}_{d}\right]$ represent two possible branches of $\tilde{t}_{\mathbf{k}}$ that satisfy the constraints for given $\bar{p}_{\mathbf{k}}$ and $\bar{q}_{d}$. The time evolutions of $\bar{p}_{\mathbf{k}}$ an $\bar{q}_{d}$ can be determined by eq. (6.19) and eq. (6.21),

$$
\begin{aligned}
& \partial_{\tau} \bar{q}_{d}=\frac{2}{\bar{q}_{d}}\left(\beta-\frac{2}{L} \sum_{\mathbf{k}} \tilde{t}_{\mathbf{k}} \bar{p}_{\mathbf{k}}\right) \\
& \partial_{\tau} \bar{p}_{\mathbf{k}}=8 \tilde{t}_{\mathbf{k}} \bar{p}_{\mathbf{k}}+\frac{8}{\bar{q}_{d}^{2}}\left(\tilde{t}_{\mathbf{k}} \bar{p}_{\mathbf{k}}-\frac{1}{L} \sum_{\mathbf{k}^{\prime}} \tilde{t}_{\mathbf{k}^{\prime}} \bar{p}_{\mathbf{k}^{\prime}}\right) \bar{p}_{\mathbf{k}} .
\end{aligned}
$$

The metric in eq. (5.19), which is independent of $\mathbf{r}$, becomes (see appendix F)

$$
-\delta g^{\mu \nu}=4 \tilde{\alpha}\left(\frac{\partial \bar{U}_{\mathbf{k}}}{\partial k_{\mu}} \frac{\partial \bar{U}_{\mathbf{k}}}{\partial k_{\nu}}+\bar{U}_{\mathbf{k}} \frac{\partial^{2} \bar{U}_{\mathbf{k}}}{\partial k_{\mu} \partial k_{\nu}}\right)_{\mathbf{k}=0} .
$$

By using eq. (6.11), eq. (7.17) can be written as

$$
-\mathcal{S} g^{\mu \nu}=-\left.\frac{4}{\tilde{\alpha}} \frac{1}{\left(\bar{p}_{\mathbf{k}}+\bar{q}_{d}^{2}\right)^{3}} \frac{\partial^{2} \bar{p}_{\mathbf{k}}}{\partial k_{\mu} \partial k_{\nu}}\right|_{\mathbf{k}=0} .
$$

Here we use eq. (7.9) and $\left.\frac{\partial \bar{p}_{\mathbf{k}}}{\partial k_{\mu}}\right|_{\mathbf{k}=0}=0$ in the presence of the inversion symmetry. This fixes the signature of spacetime upto the overall sign. Due to the emergent rotational symmetry, one can write

$$
g^{\mu \nu}(\tau)=a(\tau)^{-1} \delta^{\mu \nu},
$$

where $a(\tau)$ is the scale factor. With the choice of the positive signature for the spatial metric $(a>0)$, the signature of time is given by

$$
\mathcal{S}=\operatorname{sgn}\left(\left.\frac{1}{\tilde{\alpha}\left(\bar{p}_{0}+\bar{q}_{d}^{2}\right)^{3}} \frac{\partial^{2}}{\partial k_{1}^{2}} \bar{p}_{\mathbf{k}}\right|_{\mathbf{k}=0}\right),
$$


and the inverse of the scale factor becomes

$$
a^{-1}=\left|\frac{4}{\tilde{\alpha}} \frac{1}{\left(\bar{p}_{0}+\bar{q}_{d}^{2}\right)^{3}} \frac{\partial^{2}}{\partial k_{1}^{2}} \bar{p}_{\mathbf{k}}\right|_{\mathbf{k}=0} \mid .
$$

Due to the rotational symmetry, one can use any spatial direction to define $\delta$ and $a$ in eq. (7.20) and eq. (7.21).

According to eq. (7.21), the proper size of the emergent space becomes smaller for $\bar{p}_{\mathbf{k}}$ that varies more sharply in the momentum space. This can be understood intuitively. $\bar{p}_{\mathbf{k}}$ that changes sharply in the momentum space leads to $\bar{p}_{\mathbf{r}, \mathbf{r}^{\prime}}$ that decays slowly in $\mathbf{r}-\mathbf{r}^{\prime}$. The slowly decaying collective variables in the real space creates entanglement bonds that connect sites that are far from each other in coordinate. The long-ranged entanglement bonds bring those sites close in physical distance because they become strongly coupled under the relatively local Hamiltonian. This results in the decrease of the scale factor of space.

For $\tilde{\alpha}>0$ and $\bar{p}_{0}+\bar{q}_{d}^{2}>0$, the signature of time is given by the sign of $\frac{\partial^{2}}{\partial k_{1}^{2}} \bar{p}_{\mathbf{k}}$ at $\mathbf{k}=0$. The signature changes sign when the collective variable undergoes a Lifshitz transition at which the second derivative of $\bar{p}_{\mathbf{k}}$ changes sign. This can happen if $\bar{p}_{\mathbf{k}}$ becomes flat as a function of $\mathbf{k}$ either locally near $\mathbf{k}=0$ or globally at all $\mathbf{k}$. If $\bar{p}_{\mathbf{k}}$ becomes flat at all $\mathbf{k}$, we call the transition a global Lifshitz transition. If only the second derivative of $\bar{p}_{\mathbf{k}}$ vanishes without a global Lifshitz transition, we call it a local Lifshitz transition. At both local and global Lifshitz critical points, $g^{\mu \nu}$ vanishes, and the scale factor of the space diverges. This can be understood intuitively. When the second derivative of the collective variable vanishes, the dispersion of the collective variables becomes flat near $\mathbf{k}=0$ in the momentum space. As the band becomes flatter in the momentum space, $p_{\mathbf{r}, \mathbf{r}^{\prime}}$ and $t^{\mathbf{r}, \mathbf{r}^{\prime}}$ decay faster as a function of $\mathbf{r}-\mathbf{r}^{\prime}$ in real space. This, in turn, reduces inter-site entanglement, which results in an increase in the proper distance in space. The proper distance between two given points in the manifold increases as the collective variables lose dispersion in $\mathbf{k}$. If the band becomes completely flat, the collective variables become ultra-local, and the state becomes unentangled to the leading order in $1 / N$. This is a fragmented space [39, 51]. According to eq. (7.21), the proper volume of space diverges as far as its second derivative vanishes at $\mathbf{k}=0$ with or without a global flattening. At local Lifshitz critical points, the scale factor of the universe diverges although there still exists non-zero inter-site entanglement mediated by higher order $\mathbf{k}$ dependence of $\bar{p}_{\mathbf{k}}$. In this case, the non-trivial spatial entanglement is not encoded in the metric but in higher spin fields. This shows that the metric carries only a partial information on the pattern of entanglement. The full structure of entanglement is encoded in the complete set of collective variables. It is also these higher spin fields that carry the information that the continuous rotational symmetry is spontaneously broken to a discrete symmetry by the hypercubic lattice. Colloquially speaking, the pattern of entanglement fixes the emergent geometry, but not the other way around.

\subsection{Numerical solution}

Collective variables with different $\mathbf{k}$ remain coupled with each other through $\langle\bar{s}\rangle$ in eqs. (7.5)-(7.8). This makes it hard to solve the equations of motion in a closed form. In 


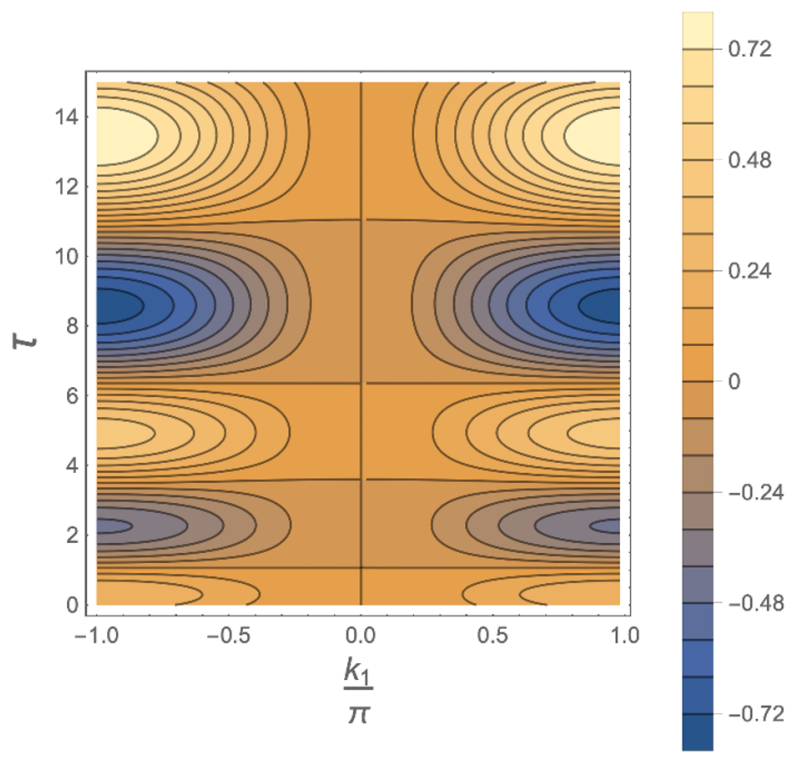

Figure 9. A contour plot of $\bar{p}_{\mathbf{k}}(\tau)$ in the plane of $\tau$ and $\mathbf{k}=\left(k_{1}, 0,0\right)$.

order to gain some insight, we first solve the equations of motion numerically for a finite system with $L=10^{6}$ and $N=\infty$. We choose the Hamiltonian with $\tilde{\alpha}=1$. For an initial state, we consider a state which has a three-dimensional local structure with the discrete translation, the $\frac{\pi}{2}$-rotation and the reflection symmetry of the cubic lattice. We choose $\bar{p}_{\mathbf{r}^{\prime}, \mathbf{r}}(0)$ that is non-zero only for nearest neighbour $\mathbf{r}^{\prime}$ and $\mathbf{r}$. In the momentum space, this gives

$$
\bar{p}_{\mathbf{k}}(0)=\bar{p}_{d}-\epsilon\left(\cos \left(k_{1}\right)+\cos \left(k_{2}\right)+\cos \left(k_{3}\right)\right),
$$

where $\bar{p}_{d}$ represents the ultra-local part of $\bar{p}_{\mathbf{r}^{\prime}, \mathbf{r}}(0)$, and $\epsilon$ determines the strength of the nearest neighbour entanglement bonds. The initial conditions for $\tilde{t}_{\mathbf{k}}$ and $\bar{s}_{\mathbf{k}}$ are fixed by the constraints with $\tilde{t}_{k}(0)=T_{+}\left[\bar{p}_{\mathbf{k}}(0), \bar{q}_{d}(0)\right]$ and $\beta=0.1$ in eq. (7.12) and eq. (7.13). The numerical results that follow is obtained for $\bar{p}_{d}=1, \bar{q}_{d}(0)=1$ and $\epsilon=0.1$.

In figure 9 , we show the evolution of $\bar{p}_{\mathbf{k}}(\tau)$ as a function of $\tau$ along one direction of $\mathbf{k}$. At $\tau=0, \bar{p}_{\mathbf{k}}$ is convex near $\mathbf{k}=0$. As $\tau$ increases, the $\mathbf{k}$ dependence becomes weaker, and $\bar{p}_{\mathbf{k}}$ becomes flatter as a function of $\mathbf{k}$. At a critical time $\tau_{1}^{*} \approx 1.065, \bar{p}_{\mathbf{k}}$ becomes independent of $\mathbf{k}$ and completely flat at all $\mathbf{k}$. As time increases further, $\bar{p}_{\mathbf{k}}$ becomes concave in $\mathbf{k}$. This is a global Lifshitz transition in which the second derivative of $\bar{p}_{\mathbf{k}}$ at $\mathbf{k}=0$ flips the sign. At the critical point, the band becomes globally dispersionless. The evolution of $\bar{p}_{\mathbf{k}}$ in $\tau$ near the critical time is shown in figure 10(a). As $\tau$ increases further, $\bar{p}_{\mathbf{k}}$ undergoes a second Lifshitz transition at $\tau_{2}^{*} \approx 3.6$. This time, the Lifshitz transition is local: $\frac{\partial^{2}}{\partial k_{\mu} \partial k_{\nu}} \bar{p}_{\mathbf{k}}$ at $\mathbf{k}=0$ changes sign from negative to positive while the band does not become globally flat. The profile of $\bar{p}_{\mathbf{k}}$ near the local Lifshitz transition is shown in figure 10(b). As time keeps increasing, the second set of global and local Lifshitz transitions occur at $\tau_{3}^{*} \approx 6.365$ and $\tau_{4}^{*} \approx 11.035$, respectively as is shown in figure 10(c) and figure 10(d). This evolution 


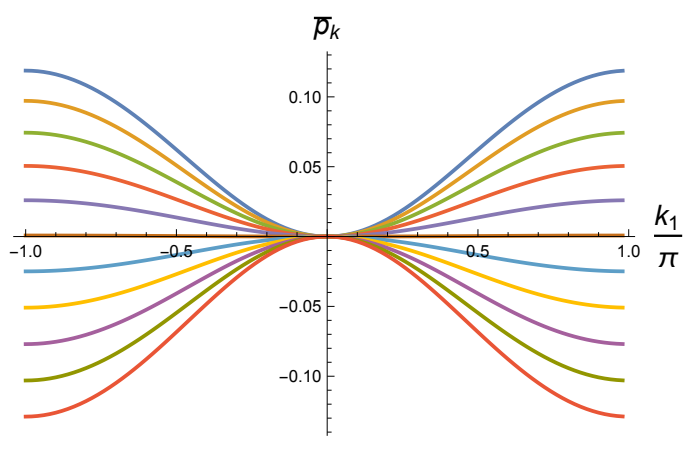

(a)

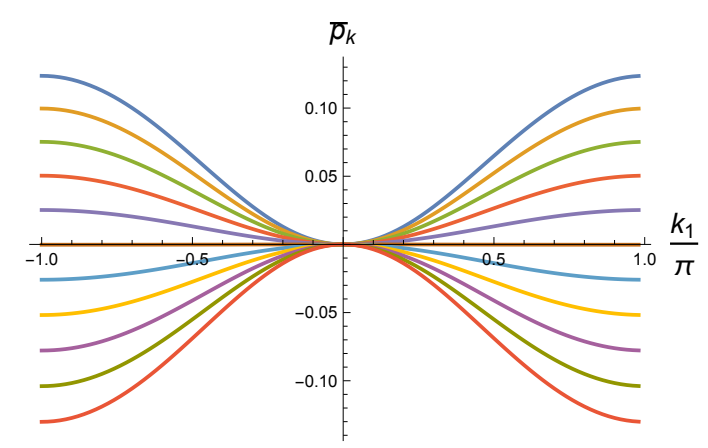

(c)

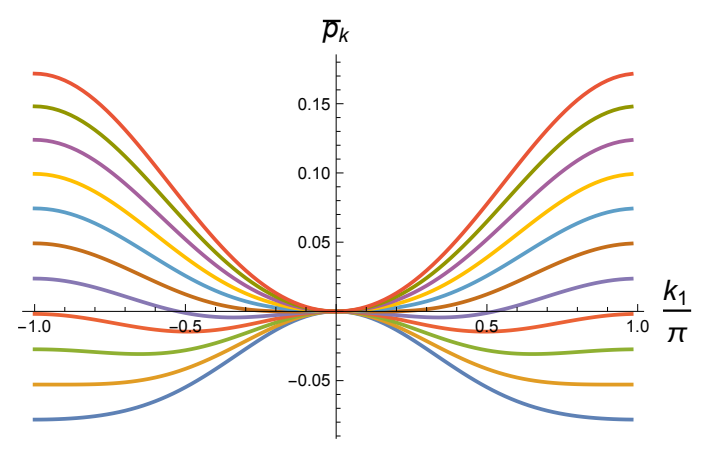

(b)

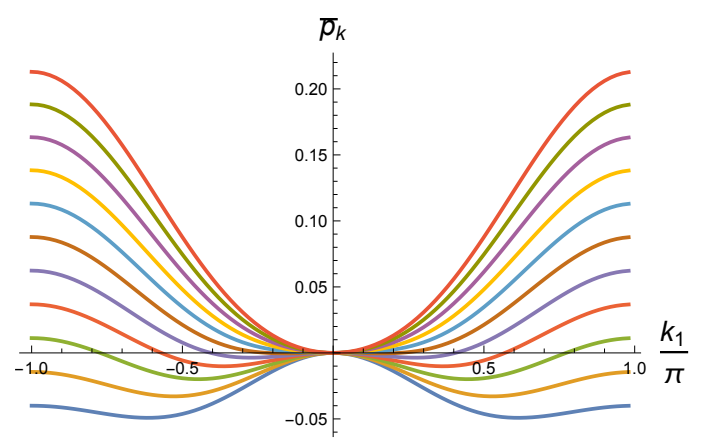

(d)

Figure 10. $\bar{p}_{\mathbf{k}}(\tau)$ plotted as a function of $\mathbf{k}=\left(k_{1}, 0,0\right)$ at different time slices. (a) From the top to bottom curves, $\tau$ changes from 0.815 to 1.315 in the step size of 0.05 . At $\tau=\tau_{1}^{*} \approx 1.065$, the collective variable that describes the pattern of entanglement undergoes a global Lifshitz transition, where $\bar{p}_{\mathbf{k}}$ becomes flat at all $\mathbf{k}$. (b) From the bottom to top curves, $\tau$ changes from 3.35 to 3.85 in the step size of 0.05 . At $\tau=\tau_{2}^{*} \approx 3.6$, the collective variabl undergoes a local Lifshitz transition, where the second derivative of $\bar{p}_{\mathbf{k}}$ vanishes at $\mathbf{k}=0$ while higher derivatives remain non-zero. (c) From the top to bottom curves, $\tau$ changes from 6.115 to 6.615 in the step size of 0.05 . At $\tau=\tau_{3}^{*} \approx 6.365$, the collective variable undergoes the second global Lifshitz transition. (d) From the bottom to top curves, $\tau$ changes from 10.785 to 11.285 in the step size of 0.05 . At $\tau=\tau_{4}^{*} \approx 11.035$, the collective variable undergoes the second local Lifshitz transition.

of $\bar{p}_{\mathbf{k}}$ near the second global Lifshitz transition is almost identical to the evolution near the first transition. This 'universality' can be understood from an analytic solution that is valid near the global Lifshitz transitions. This will be discussed in the following section.

Although the profile of $\bar{q}_{d}(\tau)$ is not exactly periodic, it follows an oscillatory pattern. In figure $11(\mathrm{a})$, we plot $\bar{q}_{d}(\tau)$ as a function of $\tau$. During one oscillation of $\bar{q}_{d}$, the collective variable undergoes four Lifshitz transitions, alternating between global and local Lifshitz transitions as is shown in figure 9. The global Lifshitz transitions coincide with the points at which $\bar{q}_{d}$ vanishes. This concurrence will be explained through analytic solutions in the next section. At the Lifshitz critical points (either global or local), the scale factor diverges, and the signature of time changes as is shown in figure 11(b) and figure 11(c). During the periods in which $\delta<0$, we have de Sitter-like spacetimes with the Lorentzian 


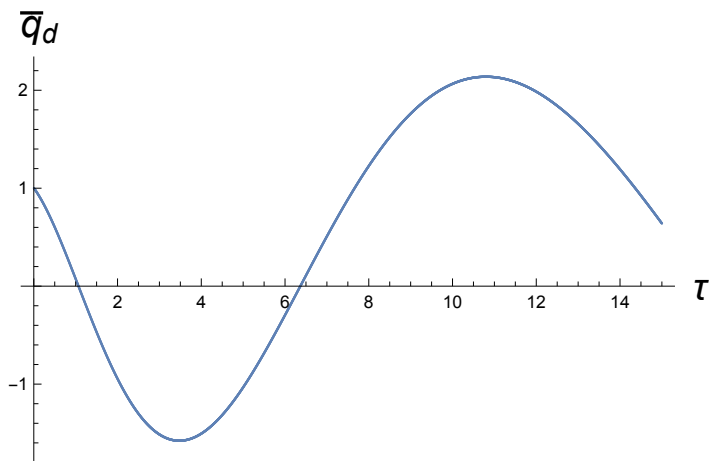

(a)

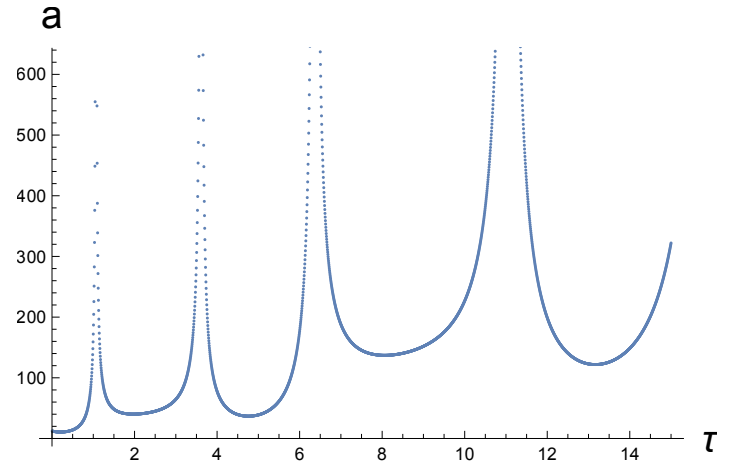

(b)

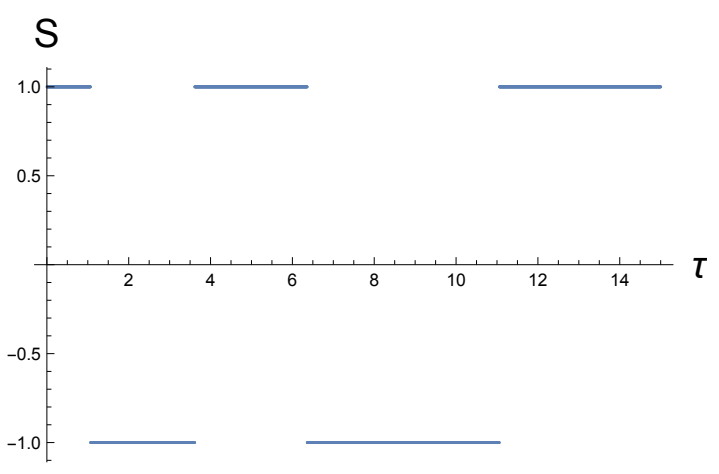

(c)

Figure 11. $\bar{q}_{d}, a, \mathcal{S}$ plotted as a function of $\tau$. Here, $\bar{q}_{d}$ is the scalar defined in eq. (6.16). $a$ is the scale factor of the space defined by $g_{\mu \nu}=a \delta_{\mu \nu}$ for the space with the translational symmetry and the reflection symmetry. $\mathcal{S}$ is the signature of time.

signature [52]. Within one epoch of the de Sitter spacetime, the universe initially starts with the infinite size, contracts to reach a minimum size, and bounces back to expand to the infinite size again. In one epoch, $\bar{q}_{d}$ varies monotonically, and can be used as a clock. Multiple de Sitter-like spacetimes are connected by four dimensional Euclidean spaces.

\subsection{Analytic solution}

The global Lifshitz transitions occur when $\bar{q}_{d}$ vanishes. When $\bar{q}_{d}$ is small, the equation of motion can be solved analytically using $\bar{q}_{d}$ as a small parameter. In this section, we present the analytical solution valid when $\bar{q}_{d}$ is small. The analytic solution confirms the features observed in the numerical solution.

In the small $\bar{q}_{d}$ limit, eqs. (7.14) and (7.12) becomes

$$
\begin{aligned}
T_{ \pm}\left[\bar{p}_{\mathbf{k}}, \bar{q}_{d}\right] & =\frac{\beta}{2 \bar{p}_{\mathbf{k}}} \pm \frac{\gamma}{4 \bar{p}_{\mathbf{k}}^{3 / 2}} \bar{q}_{d}+O\left(\bar{q}_{d}^{2}\right), \\
\bar{s}_{\mathbf{k}} & =\mp \frac{\gamma}{2 \bar{p}_{\mathbf{k}}^{1 / 2}}+O\left(\bar{q}_{d}\right) .
\end{aligned}
$$


To the leading order in $\bar{q}_{d}$, eq. (7.15) and eq. (7.16) become

$$
\begin{aligned}
& \partial_{\tau} \bar{q}_{d}=\mp \gamma<\bar{p}^{-\frac{1}{2}}> \\
& \partial_{\tau} \bar{p}_{\mathbf{k}}= \pm \frac{2 \gamma}{\bar{q}_{d}}\left(\bar{p}_{\mathbf{k}}^{-\frac{1}{2}}-<\bar{p}^{-\frac{1}{2}}>\right) \bar{p}_{\mathbf{k}}
\end{aligned}
$$

for branches $T_{+}\left[\bar{p}_{\mathbf{k}}, \bar{q}_{d}\right]$ and $T_{-}\left[\bar{p}_{\mathbf{k}}, \bar{q}_{d}\right]$, respectively, where $<\bar{p}^{-\frac{1}{2}}>\equiv \frac{1}{L} \sum_{\mathbf{k}} \bar{p}_{\mathbf{k}}^{-\frac{1}{2}}$. If $\bar{q}_{d}(\tau)=$ 0 at $\tau=\tau^{*}$, the solution to eq. (7.24) is given by

$$
\bar{q}_{d}(\tau)=\mp \gamma<\bar{p}\left(\tau^{*}\right)^{-\frac{1}{2}}>\left(\tau-\tau^{*}\right)+O\left(\left(\tau-\tau^{*}\right)^{2}\right) .
$$

To keep track of momentum dependece of $\bar{p}_{\mathbf{k}}$, it is convenient to consider the equation of motion for $\delta \bar{p}_{\mathbf{k}}=\bar{p}_{\mathbf{k}}-\bar{p}_{0}$. To the leading order in $\left(\tau-\tau^{*}\right)$ and $\delta \bar{p}_{\mathbf{k}}$, the equation of motion for $\delta \bar{p}_{\mathbf{k}}$ is given by

$$
\partial_{\tau} \delta \bar{p}_{\mathbf{k}}=\mp \gamma\left(2<\bar{p}^{-\frac{1}{2}}>-\bar{p}_{0}^{-\frac{1}{2}}\right) \frac{\delta \bar{p}_{\mathbf{k}}}{\bar{q}_{d}}
$$

To the leading order in $\left(\tau-\tau^{*}\right)$, the solutions to eq. (7.27) is obtained to be

$$
p_{\mathbf{k}}(\tau)=\bar{p}_{0}(\tau)+\left(\tau-\tau^{*}\right) f_{\mathbf{k}},
$$

where $f_{\mathbf{k}}$ is a function of $\mathbf{k}$ that is determined by matching $\bar{p}_{\mathbf{k}}(\tau)$ away from $\tau=\tau^{*}$. In eq. (7.28) we use the fact that $\left\langle p\left(\tau^{*}\right)^{-\frac{1}{2}}>=\bar{p}_{0}\left(\tau^{*}\right)^{-\frac{1}{2}}\right.$, which holds because $\delta \bar{p}_{\mathbf{k}}\left(\tau^{*}\right)=0$. Eq. (7.28) implies that near $\tau=\tau^{*}$ the momentum dependence in $\delta \bar{p}_{\mathbf{k}}$ linearly vanishes in $\tau-\tau^{*}$ so that $\bar{p}_{\mathbf{k}}$ becomes independent of $\mathbf{k}$ at $\tau^{*}$. For $\tilde{\alpha}>0$ and $\bar{p}_{0}\left(\tau^{*}\right)>0$, the signature and the scale factor of the universe are given by

$$
\begin{aligned}
\mathcal{S} & =\operatorname{sgn}\left(\left(\tau-\tau^{*}\right) \frac{\partial^{2}}{\partial k_{1}^{2}} f_{\mathbf{k}}\right), \\
a(\tau) & =\left|\frac{1}{\left.\frac{4}{\tilde{\alpha} \bar{p}_{0}\left(\tau^{*}\right)^{3}} \frac{\partial^{2}}{\partial k_{1}^{2}} f_{\mathbf{k}}\right|_{\mathbf{k}=0}\left(\tau-\tau^{*}\right)}\right| .
\end{aligned}
$$

This explains why the signature changes and the scale factor diverges at the global Lifshitz critical points.

Near the local Lifshitz transitions, the collective variables varies in time such that only the second derivative in $\mathbf{k}$ vanishes linearly in time as

$$
p_{\mathbf{k}}(\tau)=\bar{p}_{0}(\tau)+B\left(\tau-\tau^{*}\right) \mathbf{k}^{2}+O\left(\mathbf{k}^{4}\right)
$$

where $B$ is a constant and $\tau^{*}$ is the critical point. This also causes the change in the signature of time, and the divergence of the scale factor as $a \propto \frac{1}{\left|\tau-\tau^{*}\right|}$ at the local Lifshitz critical points. The emergent geometry is insensitive to the terms that are quartic and higher order in $k$, and contains only a partial information on the pattern of entanglement of the microscopic degree of freedom. 


\subsection{Emergent Lorentz symmetry}

Does the spacetime with the Lorentzian signature respect the Lorentz symmetry? We answer this question for the saddle point solution of the form in eq. (7.1) with the discrete translational symmetry. The full saddle-point solution is not invariant under the Lorentz transformation because the terms that are quartic or higher in $\mathbf{k}$ in eq. (7.30) break the rotational symmetry. Nonetheless, the full rotational symmetry is restored in the small $\mathbf{k}$ limit as the higher order terms are suppressed. Since the translational and rotational symmetries emerge in the long wavelength limit, we only need to check the boost to see whether the full Lorentz symmetry emerges.

We consider a boost generated by

$$
K_{\vec{\zeta}}=\mathscr{G}_{\eta}+\mathscr{H}_{\rho}+\mathscr{T}_{\tilde{o}_{L}}
$$

Here $\mathscr{G}$ and $\mathscr{H}$ are the generators of the generalized spatial diffeomorphism and time translation defined in eqs. (4.12) and (4.11), respectively. $\eta$ and $\rho$ are the shift and lapse tensors given by

$$
\begin{aligned}
\eta_{i}^{j} & =\frac{\varepsilon}{2}\left(\delta_{r_{j}-r_{i}, \tau \zeta}-\delta_{r_{j}-r_{i},-\tau \zeta}\right), \\
\rho_{i j} & =\varepsilon\left(\vec{r}_{i} \cdot \vec{\zeta}\right) \delta_{i j},
\end{aligned}
$$

where $\varepsilon$ is an infinitesimal parameter. $\mathscr{G}_{\eta}$ generates the time-dependent spatial translation by $\varepsilon \tau \vec{\zeta}$, where $\vec{\zeta}$ is a spatial vector. $\mathscr{H}_{\rho}$ generates the space-dependent temporal translation by $\varepsilon \vec{r} \cdot \vec{\zeta} \equiv \varepsilon g_{\mu \nu} r^{\mu} \zeta^{\nu}$. Combined, they generate the boost along $\vec{\zeta}$ in the continuum limit. The boost is augmented with an $\mathrm{O}(L) \subset \mathrm{O}(M)$ flavour transformation,

$$
\mathscr{T}_{\tilde{o}_{L}}=\operatorname{tr}\left\{q s \tilde{o}_{L}\right\}
$$

where $\tilde{o}_{L}$ is an $L \times L$ anti-symmetric matrix. Eq. (7.34) is independent of $t_{c}$ and $p_{c}$ because they are singlets under the $\mathrm{O}(L)$ flavour symmetry. The flavour rotation is included in eq. (7.34) because some solutions are invariant under a combination of the boost and an internal flavour rotation, but not under the boost alone. From now on, we refer to the combined transformation as boost.

The boost is a part of the full spacetime gauge symmetry and the global symmetry, and the gauge constraints remain satisfied under the boost. Since $s$ and $t_{c}$ are determined from $q, p_{c}$ through the gauge constraints in eqs. (7.12) and (7.13), we only need to check how $y, v, p_{c}$ and $q$ transform under the boost. In order to understand the isometry of the emergent spacetime, we focus on the shift vector, the lapse function and the spatial metric, which together form the spacetime metric. Since the spatial metric is determined from $p_{c}$ and $q$ through eq. (7.18), we consider the transformations of $p_{c}, q$, the shift vector $\left(\xi^{\mu}\right)$ and the lapse function $(\theta)$ generated by the boost. It is straightforward to show that the boost generates the following transformations (see appendix $\mathrm{G}$ for derivation),

$$
\begin{aligned}
\delta \xi^{\mu} & =\varepsilon[1+\delta] \zeta^{\mu}, & & \delta \theta=0, \\
\delta p_{c} & =4\left(p_{c} \tilde{t}_{c} \rho+\rho \tilde{t}_{c} p_{c}\right), & \delta q & =2 s^{T} \rho,
\end{aligned}
$$


where $\tilde{o}_{L}=-\eta$ is chosen. The shift vector and the lapse function are invariant under the boost for the spacetime with the Lorentzian signature $(\delta=-1) \cdot p_{c}$ and $q$ remain invariant only if $\tilde{t}_{c}=0$ and $s=0$. It is not surprising that the Lorentz symmetry is broken by non-zero $\tilde{t}_{c}$ and $s$ because they are odd under the time-reversal symmetry (see eq. (4.23)). What is less trivial is the fact that the Lorentz symmetry indeed emerges as an isometry of the spacetime as far as the time-reversal symmetry are kept along with the discrete translational and rotational symmetry.

The saddle-point solution that follows from a generic initial condition is not static, and does not respect the Lorentz symmetry. However, Lorentz-invariant static solutions can also be found. For example, with the choice of $\tilde{\alpha}=4$,

$$
\begin{aligned}
y & =0, \quad v=I, \\
s & =0, \quad q=0, \\
\tilde{t}_{c} & =0, \quad p_{c}=\frac{1}{2}\left[\bar{p}_{d}-\epsilon\left(\cos \left(k_{1}\right)+\cos \left(k_{2}\right)+\cos \left(k_{3}\right)\right)\right],
\end{aligned}
$$

with $\bar{p}_{d}>0$ satisfies the equation of motion in eq. (6.7). This is a static solution with the time-reversal symmetry. For $\epsilon<0$, the emergent spacetime metric is invariant under the Lorentz symmetry. This gives rise to the Minkowski spacetime in the continuum. For $\epsilon>0$, the resulting spacetime is Euclidean. The Minkowski spacetime is a fine-tuned solution within the present theory.

\subsection{Effective theory}

In this section, we derive the effective theory that describes fluctuations of the collective variables around the general time-dependent saddle-point configuration. The microscopic theory does not have a fixed background. However, the saddle-point configuration of the collective variables provides the spacetime on which their fluctuations propagate. We expand the collective variables around the saddle point in eq. (7.1),

$$
\begin{aligned}
p_{c}^{\prime} & =p_{c}-\frac{\bar{p}}{2}, \quad t_{c}^{\prime}=t_{c}-\frac{i}{8} p_{c}^{-1}-\tilde{t}, \\
q^{\prime} & =q-\bar{q}_{d} I, \quad s^{\prime}=s-\bar{s},
\end{aligned}
$$

where $\bar{p}, \tilde{t}, \bar{q}_{d}, \bar{s}$ satisfy the saddle-point equations in eqs. (7.5)-(7.8). Here $t_{c}^{\prime}$ represents the fluctuation of $t_{c}-\frac{i}{8} p_{c}^{-1}$ whose saddle point value is $\tilde{t}$ (see eq. (6.22)). With the shift and lapse tensors given by eqs. (6.14) and (6.17), the quadratic action for the fluctuations of the collective variables is written as

$$
S_{2}=N \int_{0}^{\infty} d \tau \operatorname{tr}\left\{s^{\prime} \partial_{\tau} q^{\prime}+t_{c}^{\prime} \partial_{\tau} p_{c}^{\prime}-\mathscr{H}_{2}-\mathscr{G}_{2}\right\} .
$$

Here boundary terms are not shown. The quadratic Hamiltonian and the $\operatorname{SL}(L, \mathbb{R})$ generator are given by

$$
\begin{aligned}
\mathscr{H}_{2}= & s^{\prime} s^{\prime T}+\tilde{\alpha} q^{\prime} \bar{U}^{2} q^{\prime T}+\tilde{\alpha}\left(2 \bar{U} U^{\prime} Q^{\prime}+\bar{Q} U^{\prime} U^{\prime}\right) \\
& +\sum_{c=1,2}\left[4 \tilde{t}\left(t_{c}^{\prime} p_{c}^{\prime}+p_{c}^{\prime} t_{c}^{\prime}\right)+2 \bar{p} t_{c}^{\prime} t_{c}^{\prime}+\frac{1}{2} \frac{1}{\bar{p}} p_{c}^{\prime} \frac{1}{\bar{p}} p_{c}^{\prime} \frac{1}{\bar{p}}\right], \\
\mathscr{G}_{2}= & -\frac{2}{\bar{q}_{d}}(\bar{s}-<\bar{s}>)\left(s^{\prime} q^{\prime}+2 \sum_{c=1,2} t_{c}^{\prime} p_{c}^{\prime}\right) .
\end{aligned}
$$


$\bar{U}=\bar{s} \bar{s}^{T}+4 \tilde{t} \bar{p} \tilde{t}+\frac{1}{4} \frac{1}{\bar{p}}$ and $\bar{Q}=\left(\bar{q}_{d}^{2} I+\bar{p}\right)$ represent the saddle-point of $U$ and $Q$ defined in eq. (4.21). $U^{\prime}$ and $Q^{\prime}$ represent fluctuations of $U$ and $Q$ which are linear superpositions of $t_{c}^{\prime}, p_{c}^{\prime}, s^{\prime}$ and $q^{\prime}: U^{\prime}=s^{\prime} \bar{s}+\bar{s} s^{\prime T}+\sum_{c=1,2}\left[2 t_{c}^{\prime} \bar{p} \tilde{t}+4 \tilde{t} p_{c}^{\prime} \tilde{t}+2 \tilde{t} \bar{p} t_{c}^{\prime}-\frac{1}{4} \frac{1}{\bar{p}} p_{c}^{\prime} \frac{1}{\bar{p}}\right]$ and $Q^{\prime}=$ $\bar{q}_{d}\left(q^{\prime}+q^{\prime}\right)+\sum_{c=1,2} p_{c}^{\prime}$. The shift and lapse tensors are fixed to be eq. (6.14) and eq. (6.17), respectively. In eq. (7.39), we use $y=-\frac{2}{\bar{q}_{d}}(\bar{s}-<\bar{s}>)$ on the constraint hypersurface. Since $U^{\prime}$ and $Q^{\prime}$ depend on $t_{1}^{\prime}$ and $t_{2}^{\prime}$ (and $p_{1}^{\prime}$ and $p_{2}^{\prime}$ ) only through their symmetric combinations, it is useful to introduce

$$
\begin{array}{ll}
t_{+}^{\prime}=\frac{t_{1}^{\prime}+t_{2}^{\prime}}{\sqrt{2}}, & t_{-}^{\prime}=\frac{t_{1}^{\prime}-t_{2}^{\prime}}{\sqrt{2}}, \\
p_{+}^{\prime}=\frac{p_{1}^{\prime}+p_{2}^{\prime}}{\sqrt{2}}, & p_{-}^{\prime}=\frac{p_{1}^{\prime}-p_{2}^{\prime}}{\sqrt{2}}
\end{array}
$$

to rewrite $\mathscr{H}_{2}$ and $\mathscr{G}_{2}$ as

$$
\begin{aligned}
\mathscr{H}_{2}= & s^{\prime} s^{\prime T}+\tilde{\alpha} q^{\prime} \bar{U}^{2} q^{\prime T}+\tilde{\alpha}\left(2 \bar{U} U^{\prime} Q^{\prime}+\bar{Q} U^{\prime} U^{\prime}\right) \\
& +\left[4 \tilde{t}\left(t_{+}^{\prime} p_{+}^{\prime}+p_{+}^{\prime} t_{+}^{\prime}\right)+2 \bar{p} t_{+}^{\prime} t_{+}^{\prime}+\frac{1}{2} \frac{1}{\bar{p}} p_{+}^{\prime} \frac{1}{\bar{p}} p_{+}^{\prime} \frac{1}{\bar{p}}\right] \\
& +\left[4 \tilde{t}\left(t_{-}^{\prime} p_{-}^{\prime}+p_{-}^{\prime} t_{-}^{\prime}\right)+2 \bar{p} t_{-}^{\prime} t_{-}^{\prime}+\frac{1}{2} \frac{1}{\bar{p}} p_{-}^{\prime} \frac{1}{\bar{p}} p_{-}^{\prime} \frac{1}{\bar{p}}\right], \\
\mathscr{S}_{2}= & -\frac{2}{\bar{q}_{d}}(\bar{s}-<\bar{s}>)\left(s^{\prime} q^{\prime}+2 t_{+}^{\prime} p_{+}^{\prime}+2 t_{-}^{\prime} p_{-}^{\prime}\right),
\end{aligned}
$$

where

$$
\begin{aligned}
U^{\prime} & =s^{\prime} \bar{s}+\bar{s} s^{\prime T}+\sqrt{2}\left[2 t_{+}^{\prime} \bar{p} \tilde{t}+4 \tilde{t} p_{+}^{\prime} \tilde{t}+2 \tilde{t} \bar{p} t_{+}^{\prime}-\frac{1}{4} \frac{1}{\bar{p}} p_{+}^{\prime} \frac{1}{\bar{p}}\right], \\
Q^{\prime} & =\bar{q}_{d}\left(q^{\prime T}+q^{\prime}\right)+\sqrt{2} p_{+}^{\prime} .
\end{aligned}
$$

$t_{ \pm}^{\prime}, p_{ \pm}^{\prime}, s^{\prime}, q^{\prime}$ are all $L \times L$ matrices which become bi-local fields in the continuum. Due to the local structure, $\tilde{t}^{\mathbf{r}_{1}, \mathbf{r}_{2}}, \bar{p}_{\mathbf{r}_{1}, \mathbf{r}_{2}}, \bar{s}^{\mathbf{r}_{1}}{ }_{\mathbf{r}_{2}}$ decay exponentially in $\mathbf{r}_{1}-\mathbf{r}_{2}$, and $\tilde{t}_{\mathbf{k}}, \bar{p}_{\mathbf{k}}, \bar{s}_{\mathbf{k}}$ are analytic functions of momentum. This guarantees that the effective theory that describes propagation of the fluctuating modes is local.

We can write the effective theory in the gradient expansion. To organize the gradient expansion, it is useful to note that $\bar{s}_{\mathbf{k}}$ and $\tilde{t}_{\mathbf{k}}$ are both determined from $\bar{p}_{\mathbf{k}}$ and $\bar{q}_{d}$ through eqs. (7.12) and (7.13). Since the spatial metric is directly related to $\bar{U}_{\mathbf{k}}$ through eq. (7.17), it is convenient to take $\bar{U}_{\mathbf{k}}$ and $\bar{q}_{d}$ as independent variables, and write $\bar{s}_{\mathbf{k}}, \tilde{t}_{\mathbf{k}}, \bar{p}_{\mathbf{k}}$ as functions of $\bar{U}_{\mathbf{k}}$ and $\bar{q}_{d}$ as

$$
\bar{s}_{\mathbf{k}}=S\left[\bar{U}_{\mathbf{k}}, \bar{q}_{d}\right], \quad \tilde{t}_{\mathbf{k}}=T\left[\bar{U}_{\mathbf{k}}, \bar{q}_{d}\right], \quad \bar{p}_{\mathbf{k}}=P\left[\bar{U}_{\mathbf{k}}, \bar{q}_{d}\right]
$$

$\bar{U}_{\mathbf{k}}$ is an analytic function of $\mathbf{k}$. In real space, $\bar{U}^{\mathbf{r}_{1}, \mathbf{r}_{2}}$ decays exponentially over the coordinate scale that corresponds to a unit proper distance. ${ }^{11}$ Consequently, $\bar{s}, \tilde{t}, \bar{p}$ all decay

\footnotetext{
${ }^{11}$ This is how the metric is defined in eq. (7.17).
} 
exponentially in real space in the same manner. Expanding $\bar{U}_{\mathbf{k}}, \bar{s}_{\mathbf{k}}, \tilde{t}_{\mathbf{k}}, \bar{p}_{\mathbf{k}}, y_{\mathbf{k}}$ in $\mathbf{k}$, we write

$$
\begin{aligned}
\bar{U}_{\mathbf{k}} & =\bar{U}^{[0]}+\bar{U}^{[2]} g^{\mu \nu} k^{\mu} k^{\nu}+O\left(k^{4}\right), \\
\bar{s}_{\mathbf{k}} & =\bar{s}^{[0]}+\bar{s}^{[2]} g^{\mu \nu} k^{\mu} k^{\nu}+O\left(k^{4}\right), \\
\tilde{t}_{\mathbf{k}} & =\tilde{t}^{[0]}+\tilde{t}^{[2]} g^{\mu \nu} k^{\mu} k^{\nu}+O\left(k^{4}\right), \\
\bar{p}_{\mathbf{k}} & =\bar{p}^{[0]}+\bar{p}^{[2]} g^{\mu \nu} k^{\mu} k^{\nu}+O\left(k^{4}\right), \\
\bar{y}_{\mathbf{k}} & =\bar{y}^{[0]}+\bar{y}^{[2]} g^{\mu \nu} k^{\mu} k^{\nu}+O\left(k^{4}\right),
\end{aligned}
$$

where $\bar{U}^{[2]}=\left.\frac{1}{8 \tilde{\alpha} \bar{U}_{\mathbf{k}}}\right|_{\mathbf{k}=0}, \bar{s}^{[0]}=S\left[\bar{U}^{[0]}, \bar{q}_{d}\right], \bar{s}^{[2]}=\left.\frac{1}{8 \tilde{\alpha} \bar{U}_{\mathbf{k}}} \frac{\partial S\left[\bar{U}_{\mathbf{k}}, \bar{q}_{d}\right]}{\partial \bar{U}_{\mathbf{k}}}\right|_{\mathbf{k}=0}, \tilde{t}^{[0]}=T\left[\bar{U}^{[0]}, \bar{q}_{d}\right], \tilde{t}^{[2]}=$ $\left.\frac{1}{8 \tilde{\alpha} \bar{U}_{\mathbf{k}}} \frac{\partial T\left[\bar{U}_{\mathbf{k}}, \bar{q}_{d}\right]}{\partial \bar{U}_{\mathbf{k}}}\right|_{\mathbf{k}=0}, \bar{p}^{[0]}=P\left[\bar{U}^{[0]}, \bar{q}_{d}\right], \bar{p}^{[2]}=\left.\frac{1}{8 \tilde{\alpha} \bar{U}_{\mathbf{k}}} \frac{\partial P\left[\bar{U}_{\mathbf{k}}, \bar{q}_{d}\right]}{\partial \bar{U}_{\mathbf{k}}}\right|_{\mathbf{k}=0}, \bar{y}^{[0]}=-\frac{2}{\bar{q}_{d}}\left(\bar{s}^{[0]}-<\bar{s}>\right)$ and $\bar{y}^{[2]}=-\left.\frac{1}{\bar{q}_{d}} \frac{1}{4 \tilde{\alpha} \bar{U}_{\mathbf{k}}} \frac{\partial S\left[\bar{U}_{\mathbf{k}}, \bar{q}_{d}\right]}{\partial \bar{U}_{\mathbf{k}}}\right|_{\mathbf{k}=0} \cdot \bar{y}_{\mathbf{k}}$ is the shift tensor in eq. (6.14) written in the momentum space. $\bar{s}^{[0]}, \bar{s}^{[2]}, \tilde{t}^{[0]}, \tilde{t}^{[2]}, \bar{p}^{[0]}, \bar{p}^{[2]}, \bar{y}^{[0]}, \bar{y}^{[2]}$ are all functions of $\bar{q}_{d}(\tau)$ and $\bar{U}^{[0]}(\tau)$. In the coordinate system in eq. (7.2), the effective theory for the propagating bi-local modes becomes

$$
\begin{aligned}
& S_{2}=N \int d \tau d \mathbf{r}_{A} d \mathbf{r}_{B}\left\{s^{\prime}\left(\mathbf{r}_{A}, \mathbf{r}_{B}\right) \partial_{\tau} q^{\prime}\left(\mathbf{r}_{B}, \mathbf{r}_{A}\right)+\sum_{\sigma= \pm} t_{\sigma}^{\prime}\left(\mathbf{r}_{A}, \mathbf{r}_{B}\right) \partial_{\tau} p_{\sigma}^{\prime}\left(\mathbf{r}_{B}, \mathbf{r}_{A}\right)\right. \\
& -s^{\prime}\left(\mathbf{r}_{A}, \mathbf{r}_{B}\right) s^{\prime}\left(\mathbf{r}_{A}, \mathbf{r}_{B}\right)-\tilde{\alpha} q^{\prime}\left(\mathbf{r}_{A}, \mathbf{r}_{B}\right)\left(\bar{U}^{[0] 2}-2 \bar{U}^{[0]} \bar{U}^{[2]} \nabla_{B}^{2}\right) q^{\prime}\left(\mathbf{r}_{A}, \mathbf{r}_{B}\right) \\
& -\sum_{\sigma= \pm}\left[4 p_{\sigma}^{\prime}\left(\mathbf{r}_{A}, \mathbf{r}_{B}\right)\left(\tilde{t}^{[0]}-\tilde{t}^{[2]} \nabla^{2}\right) t_{\sigma}^{\prime}\left(\mathbf{r}_{A}, \mathbf{r}_{B}\right)\right. \\
& +4 t_{\sigma}^{\prime}\left(\mathbf{r}_{A}, \mathbf{r}_{B}\right)\left(\tilde{t}^{[0]}-\tilde{t}^{[2]} \nabla^{2}\right) p_{\sigma}^{\prime}\left(\mathbf{r}_{A}, \mathbf{r}_{B}\right) \\
& +2 t_{\sigma}^{\prime}\left(\mathbf{r}_{A}, \mathbf{r}_{B}\right)\left(\bar{p}^{[0]}-\bar{p}^{[2]} \nabla^{2}\right) t_{\sigma}^{\prime}\left(\mathbf{r}_{A}, \mathbf{r}_{B}\right) \\
& \left.+\frac{1}{2}\left(\frac{1}{\bar{p}^{[0] 2}}+2 \frac{\bar{p}^{[2]}}{\bar{p}^{[0] 3}} \nabla_{A}^{2}\right) p_{\sigma}^{\prime}\left(\mathbf{r}_{A}, \mathbf{r}_{B}\right)\left(\frac{1}{\bar{p}^{[0]}}+\frac{\bar{p}^{[2]}}{\bar{p}^{[0] 2}} \nabla_{B}^{2}\right) p_{\sigma}^{\prime}\left(\mathbf{r}_{A}, \mathbf{r}_{B}\right)\right] \\
& -2 \tilde{\alpha} Q^{\prime}\left(\mathbf{r}_{A}, \mathbf{r}_{B}\right)\left(\bar{U}^{[0]}-\bar{U}^{[2]} \nabla^{2}\right) U^{\prime}\left(\mathbf{r}_{A}, \mathbf{r}_{B}\right)-U^{\prime}\left(\mathbf{r}_{A}, \mathbf{r}_{B}\right)\left(\frac{1}{\bar{U}[0]}+\frac{\bar{U}^{[2]}}{\bar{U}^{[0] 2}} \nabla^{2}\right) U^{\prime}\left(\mathbf{r}_{A}, \mathbf{r}_{B}\right) \\
& \left.-q^{\prime}\left(\mathbf{r}_{B}, \mathbf{r}_{A}\right)\left(\bar{y}^{[0]}-\bar{y}^{[2]} \nabla_{A}^{2}\right) s^{\prime}\left(\mathbf{r}_{A}, \mathbf{r}_{B}\right)-2 \sum_{\sigma= \pm} p_{\sigma}^{\prime}\left(\mathbf{r}_{B}, \mathbf{r}_{A}\right)\left(\bar{y}^{[0]}-\bar{y}^{[2]} \nabla^{2}\right) t_{\sigma}^{\prime}\left(\mathbf{r}_{A}, \mathbf{r}_{B}\right)\right\}
\end{aligned}
$$

up to two derivative order, where $\nabla_{A}^{2}=g^{\mu \nu} \frac{\partial^{2}}{\partial r_{A}^{\mu} \partial r_{A}^{\nu}}, \nabla_{B}^{2}=g^{\mu \nu} \frac{\partial^{2}}{\partial r_{B}^{\mu} \partial r_{B}^{\nu}}$ and $\nabla^{2}=\frac{\nabla_{A}^{2}+\nabla_{B}^{2}}{2}$. Here $\bar{Q}=\frac{1}{\tilde{\alpha} U}$ is used. In eq. (7.45), all bi-local fields depend on $\tau$. The propagating degrees of freedom are kinematically non-local, namely, bi-local in this case [53, 54]. Nonetheless, the theory is dynamically local [55] in that the theory does not allow bi-local objects to suddenly jump from one location to another location non-locally. The locality of the effective theory is guaranteed by the fact that the saddle-point configurations of collective variables decay exponentially in the relative coordinate due to the local structure. As a result, the gradient expansion is well defined. At length scale larger than the scale set by the local structure, the higher order derivative terms are negligible. 


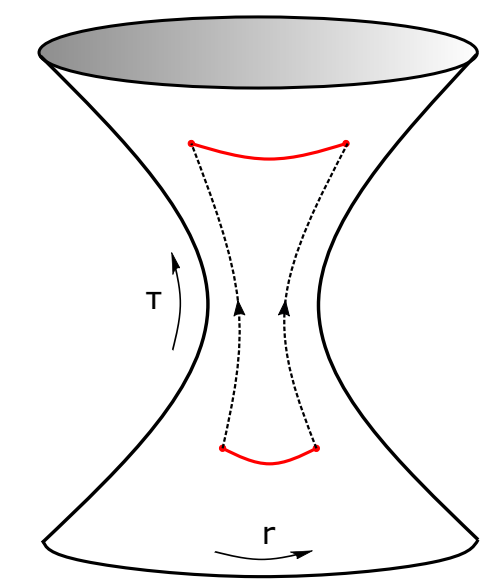

Figure 12. The hyperboloid represents the de Sitter-like spacetime that arises from the saddlepoint solution. Small fluctuations of the bi-local collective variables propagate in the spacetime, obeying the dynamics controlled by a local effective theory. The dashed lines represent the world lines of the end points of the bi-local fields.

The effective action includes terms that are cubic and higher order in the bi-local fields. For example, the cubic action takes the form of

$$
S_{3} \sim N \int d \tau d r_{A} d r_{B} d r_{C} f_{1}\left(r_{A}, r_{B}\right) f_{2}\left(r_{B}, r_{C}\right) f_{3}\left(r_{C}, r_{A}\right)
$$

where $f_{i}\left(r_{A}, r_{B}\right)$ represents one of the bi-local fields and their derivatives. Again the higher derivative terms are suppressed by the scale associated with the local structure, and the interaction terms are also local. In the large $N$ limit, the interactions are suppressed, and the bi-local fields are weakly interacting. To the leading order in $1 / N$, the bi-local objects freely propagate in the spacetime that is determined from the saddle-point configuration of the collective variables. This is illustrated in figure 12 .

While the effective theory in eq. (7.45) is local, it is not a local field theory of point-like particles. If one starts with a state with one bi-local excitation, its size can grow in time without a bound because there is no potential that confines the end points in the large $N$ limit. This is because two end points of the bi-local fields propagate freely to the leading order in the $1 / N$ expansion. The sub-leading interactions such as the one in eq. (7.46) can create bound states which behave as point particles. Only at length scales larger than the length scale of the bound state, one may use a local field theory description of point particles. However, the legnth scale of the bound state scales with $N$ in the large $N$ limit. For this reason, the present theory is far from the theory of pure gravity.

As is discussed in 4.3 , only $L(L+1)+2$ phase space variables represent physical degrees of freedom. One possible gauge choice is to determine $t_{+}^{\prime}$ and the traceless part of $s^{\prime}$ to solve the Hamiltonian constraint and the momentum constraint, respectively. Furthermore, one can fix $p_{+}^{\prime}$ and the traceless part of $q^{\prime}$ to fix the gauge associated with the Hamiltonian and the momentum constraints, respectively. This leaves $t_{-}^{\prime}, p_{-}^{\prime}$ and the trace parts of $q^{\prime}$ and $s^{\prime}$ as physical degrees of freedom. 


\section{Summary and discussion}

In this paper, we present a model for a background independent quantum gravity in which dimension, topology and geometry are all dynamical. The fundamental degree of freedom is a rectangular matrix. Matrix elements within each column define a local Hilbert space. The pattern of entanglement across local Hilbert spaces determines a spatial manifold if it has a local structure. A state has a local structure if it is short-ranged entangled when viewed as a state defined on a Riemannian manifold in which the column indices of the matrix is embedded. The theory does not have a manifold with a fixed dimension, and the spatial diffeomorphism of the general relativity is generalized to a larger group that includes diffeomorphism in arbitrary dimensions in the large $L$ limit. The Hamiltonian that generates a background independent dynamics is relatively local in that the effective interaction between sites is determined by the pre-existing entanglement between the sites. The generalized momentum and Hamiltonian constraints obey a first-class constraint algebra that is reduced to that of the hypersurface deformation algebra of the general relativity in a special case. Using the constraints, we express the projection of a state with a finite norm to a gauge invariant state as a path integration of collective variables that describe fluctuating spacetime. In the limit that the size of the matrix is large, the path integration can be replaced with a saddle-point that satisfies the classical equation of motion. The equation of motion is solved both numerically and analytically for a state that has a three-dimensional local structure with a translational symmetry. We obtain a solution that describes a series of $(3+1)$-dimensional de Sitter-like spacetimes with the Lorentzian signature which are bridged by 4 -dimensional Euclidean spaces in between. We find that the dynamical phase transitions at which the signature of spacetime changes are triggered by Lifshitz transitions of the collective variables that control the pattern of entanglement for the underlying matrix. The gravitational degrees of freedom can be identified as a composite of the collective variables. It is shown that the geometry encodes only a partial information on the entanglement of the underlying quantum matter. There exists nongeometric entanglement that is encoded in higher spin fields of the theory. ${ }^{12}$ The effective theory describes bi-local excitations that propagate in the spacetime formed by the condensate of the collective variables. We conclude with discussions on the connection to the dS/CFT and AdS/CFT correspondences and open problems.

\section{1 dS/CFT}

The present construction can be viewed as a non-perturbative formulation of the de Sitter space and conformal field theory (dS/CFT) correspondence [52, 56-61]. To illustrate the connection through a concrete example, let us consider the projection of a normalizable state to the gauge invariant state, $|0\rangle=\int D \Phi|\Phi\rangle$. For the semi-classical normalizable

\footnotetext{
${ }^{12}$ This might be phrased as $E P R>E R$.
} 
state given by eq. (4.5) with eq. (6.1), the projection is written as

$$
\begin{aligned}
\langle 0 \mid \chi\rangle= & \int D q D \phi D \varphi D s D t \times \\
& e^{i \operatorname{tr}\left\{N s q+t_{1}\left(\phi^{T} \phi\right)+t_{2}\left(\varphi^{T} \varphi\right)\right\}-i N \operatorname{tr}\left\{q s+p_{c} t_{c}\right\}-\frac{\sum_{i, \alpha}\left[s^{i}{ }^{-s^{i}} \alpha\right]^{2}+\sum_{c, i j}\left[t_{c}^{i j}-t_{c}^{i j}\right]^{2}}{\Delta^{2}} .} .
\end{aligned}
$$

Upon integrating over $s, t_{c}$, the projection is expressed as a matrix integration,

$$
\begin{aligned}
\langle 0 \mid \chi\rangle= & \int D q D \phi D \varphi e^{i N \operatorname{tr}\left\{s(q-q)+t_{1}\left(\frac{\phi^{T} \phi}{N}-p_{1}\right)+t_{2}\left(\frac{\varphi^{T} \varphi}{N}-p_{2}\right)\right\} \times} \times \\
& e^{-\frac{N^{2} \Delta^{2}}{4} \operatorname{tr}\left\{(q-q)^{T}(q-q)+\left(\frac{\phi^{T} \phi}{N}-p_{1}\right)^{2}+\left(\frac{\varphi^{T} \varphi}{N}-p_{2}\right)^{2}\right\}} .
\end{aligned}
$$

Suppose that $t_{c}$ and $p_{c}$ has a local structure. Namely, there exists a mapping from sites to a Riemannian manifold such that $t_{c, i j}$ and $p_{c}^{i j}$ decays exponentially in the proper distance between $r_{i}$ and $r_{j}$, where $r_{i}$ is the mapping from site $i$ to the manifold. In this case, eq. (8.2) can be viewed as the generating function of a local (non-unitary) field theory defined on the Riemannian manifold. The equivalence between eq. (8.2) and eq. (4.14) shows that the generating function of the non-unitary field theory is given by the path integration of the quantum gravity in which the de Sitter-like spacetime emerges at the saddle-point.

\subsection{AdS/CFT}

In order to make a connection with the AdS/CFT correspondence [8-10], one should consider a unitary field theory defined on a manifold with the Lorentzian signature instead of the Riemannian manifold. If $t_{c}$ and $p_{c}$ in eq. (8.2) exhibit a local structure, the local structure determines the signature of the manifold on which the field theory is defined. If there is a translational invariance, the signature of each direction in the manifold is determined by the sign of the second derivative of the bi-local fields in the momentum space. If the second derivative of the collective variable has the opposite sign in one direction compared with other directions, this gives rise to a Lorentzian metric. In this case, eq. (8.2) corresponds to the generating function of a Lorentzian field theory in the continuum limit. It is useful to continue to think of the generating function as an overlap of two wavefunctions, one given by $\Psi_{1}=1$ and the other given by $\Psi_{2}=e^{i S}$, where $S$ is the action of the Lorentzian quantum field theory [39]. The wavefunctions are defined on spacetime (not just in space). The Hamiltonian is then replaced with a generator of coarse graining. The fact that $\Psi_{1}$ is invariant under the transformation generated by the coarse graining implies that $\Psi_{1}$ is a fixed point action that is scale invariant. In particular, $\Psi_{1}=1$ represents the trivial insulating fixed point.

Just as the overlap between a gauge invariant state and a state with a finite norm is invariant under the gauge transformation generated by the Hamiltonian and momentum constraints in eq. (4.9), the generating function is invariant under an insertion of $e^{-i \hat{H} \tau}$, where $\hat{H}$ is the generator of coarse graining [39]. An evolution generated by successive applications of coarse graining gives rise to the exact Wilsonian renormalization group 
(RG) flow [62]. The exponent of $\Psi_{2}(\tau)=e^{-i \hat{H} \tau} \Psi_{2}$ represents the effective action defined at a length scale $\tau$. In the effective action, all couplings that are allowed by symmetry are generally turned on. While the flow of $\Psi_{2}(\tau)$ can be tracked in terms of the classical couplings that parameterize the renormalized action, it is more natural to view the RG flow as an evolution of the wavefunction in the vector space. In particular, one can choose a set of basis wavefunctions that spans the full space of wavefunctions. Because the set of single-trace operators generate all multi-trace operators, the basis states can be chosen so that their wavefunctions only include the single-trace operators. As a result, $\Psi_{2}(\tau)$ can be represented as a linear superposition of states whose actions include only single-trace operators. In this way, the classical Wilsonian RG flow defined in the space of all couplings is replaced with a quantum evolution of wavefunction defined in the space of the singletrace couplings [49]. The full Wilsonian renormalization group flow is projected to the space of single-trace operators at the expense of promoting the sources for the single-trace operators to dynamical variables. Since the source for the single-trace energy-momentum tensor is also promoted to dynamical variable, the path integration of the dynamical source represents a dynamical gravity in the bulk. This may eventually provide a non-perturbative formulation of quantum gravity in the anti-de Sitter space. One missing piece in the puzzle is to construct a UV finite coarse graining operator such as the one in eq. (3.20) that gives rise to a background independent gravity in the bulk.

\subsection{Open questions}

Here we list some open questions.

- Physical spectrum

In general, saddle-point configurations do not have temporal Killing vector. It would be interesting to find a saddle-point configuration that support a temporal Killing vector and extract the physical energy spectrum of the propagating modes in this theory $[63,64]$

- Local field theory of point particles

Background independent theories of quantum gravity include kinematically non-local objects. In the present theory, it is the bi-local field. The ultimate goal is to construct a background independent theory that reduces to a field theory of a small number of point-like particles at low energies. However, the present theory does not achieve this goal because point-like particles emerge only through the interactions between bilocal fields that are suppressed by $1 / N$. This is because the present theory is similar to vector models $[39,53,54,65-77]$ although the microscopic degree of freedom is a matrix. One index of the matrix is used to generate an emergent space, and the internal symmetry acts only on the remaining one index. In order to construct a quantum theory of gravity that reduces to the Einstein's general relativity with a small number of additional fields, one needs a mechanism that keeps dynamical objects finite to the leading order in $1 / N$. For this, tensor models may be a natural direction [78-81]. In tensor models with rank greater than 2 , where one index labels 
sites and the remaining indices label internal flavour (or color), one expects to have multi-local fields as emergent degree of freedom. In this case, a non-zero tension may naturally arise to keep kinematically non-local objects finite. In relation to a non-perturbative formulation of the AdS/CFT correspondence for gauge theories, we expect that the background independent coarse grainer takes a form of a relatively local tensor model with rank 3.

\section{Acknowledgments}

I thank Bianca Dittrich for discussions. The research was supported by the Natural Sciences and Engineering Research Council of Canada. Research at the Perimeter Institute is supported in part by the Government of Canada through Industry Canada, and by the Province of Ontario through the Ministry of Research and Information.

\section{A Derivation of eq. (3.21)}

Using the singular value decomposition, a symmetric matrix can be written as $v=O_{v}^{T} D_{v} O_{v}$, where $O_{v}$ is an orthogonal matrix and $D_{v}=\left(\begin{array}{ccc}d_{1} & & \\ & d_{2} & \\ & & \ddots\end{array}\right)$ is a diagonal matrix. If $v$ is non-singular, $d_{i} \neq 0$. One can then write the diagonal matrix as $D_{v}=n_{v} X_{v} S_{v} X_{v}$, where $n_{v}=\left|\prod_{i} d_{i}\right|^{\frac{1}{L}}, X_{v}=\frac{1}{\left|\prod_{i} d_{i}\right|^{\frac{1}{2 L}}}\left(\begin{array}{lll}\sqrt{\left|d_{1}\right|} & & \\ & \sqrt{\left|d_{2}\right|} & \\ & & \ddots\end{array}\right)$ and $S_{v}=\left(\begin{array}{lll}\operatorname{sgn}\left(d_{1}\right) & & \\ & \operatorname{sgn}\left(d_{2}\right) & \\ & & \ddots\end{array}\right)$.

Therefore, $v$ can be written as $v=n_{v} g_{v}^{T} S_{v} g_{v}$, where $g_{v}=X_{v} O_{v} \in \operatorname{SL}(L, \mathbb{R})$.

\section{B Non-normalizability of gauge invariant state}

In this appendix, we prove that all gauge invariant states have infinite norm with respect to the norm defined in eq. (2.1). Consider a gauge invariant wavefunction $\Psi\left(\Phi^{A}{ }_{i}\right)$ defined in the space of $\left\{\Phi^{A}{ }_{i}\right\}$. Suppose that the wavefunction has a support away from the origin in the $M L$-dimensional space. Without loss of generality, let us choose the frame such that a point in the support is given by $\Phi^{A}{ }_{i}=\Phi_{0} \delta_{1}^{A} \delta_{i}^{1}$, where $\Phi_{0}$ is a positive constant. Under the $\operatorname{SL}(L, \mathbb{R})$ transformation generated by $\hat{G}^{1}{ }_{2}+\hat{G}^{2}{ }_{1}$, the point can be mapped into any point on the hyperbola defined by $\left(\Phi_{1}^{1}\right)^{2}-\left(\Phi_{2}^{1}\right)^{2}=\Phi_{0}^{2}$ with $\Phi_{1}^{1}>0$. In order for the wavefunction to be invariant under the transformation, the wavefunction must have amplitude $\Phi_{0}$ along the entire hyperbola. Because the hyperbola is unbounded, the norm of the wavefunction is infinite. The only state which does not have support away from the origin is $\Psi\left(\Phi_{i}^{A}\right) \propto \Pi_{A, i} \delta\left(\Phi_{i}^{A}\right)$. However, this has an infinite norm as well because the wavefunction is constant in the conjugate space. 


\section{Computation of the commutator of the Hamiltonians}

The commutator between $\hat{H}_{u}$ and $\hat{H}_{v}$ can be written as a sum of two contributions,

$$
\left[\hat{H}_{u}, \hat{H}_{v}\right]=\hat{C}_{1}+\hat{C}_{2}
$$

where

$$
\begin{aligned}
& \hat{C}_{1}=-\frac{\tilde{\alpha}}{M^{2}}\left[\operatorname{tr}\left\{\hat{\Pi} \hat{\Pi}^{T} u\right\}, \operatorname{tr}\left\{\hat{\Pi} \hat{\Pi}^{T} \hat{\Phi}^{T} \hat{\Phi} \hat{\Pi} \hat{\Pi}^{T} v\right\}\right]+\frac{\tilde{\alpha}}{M^{2}}\left[\operatorname{tr}\left\{\hat{\Pi} \hat{\Pi}^{T} v\right\}, \operatorname{tr}\left\{\hat{\Pi} \hat{\Pi}^{T} \hat{\Phi}^{T} \hat{\Phi} \hat{\Pi} \hat{\Pi}^{T} u\right\}\right], \\
& \hat{C}_{2}=\frac{\tilde{\alpha}^{2}}{M^{4}}\left[\operatorname{tr}\left\{\hat{\Pi} \hat{\Pi}^{T} \hat{\Phi}^{T} \hat{\Phi} \hat{\Pi} \hat{\Pi}^{T} u\right\}, \operatorname{tr}\left\{\hat{\Pi} \hat{\Pi}^{T} \hat{\Phi}^{T} \hat{\Phi} \hat{\Pi} \hat{\Pi}^{T} v\right\}\right] .
\end{aligned}
$$

From eq. (3.26) we obtain

$$
\hat{C}_{1}=-\frac{4 i \tilde{\alpha}}{M^{2}} \operatorname{tr}\left\{\left(\hat{\Pi} \hat{\Pi}^{T} u \hat{\Pi} \hat{\Pi}^{T} v-\hat{\Pi} \hat{\Pi}^{T} v \hat{\Pi} \hat{\Pi}^{T} u\right) \hat{\mathbf{G}}\right\} .
$$

Generators of $\operatorname{GL}(L, \mathbb{R})$ can be decomposed into generators of $\operatorname{SL}(L, \mathbb{R})$ and a global dilatation generator in eq. (3.8). The contribution of the latter vanishes due to the cyclic property of the trace. This gives

$$
\hat{C}_{1}=-\frac{4 i \tilde{\alpha}}{M^{2}} \operatorname{tr}\left\{\left(\hat{\Pi} \hat{\Pi}^{T} u \hat{\Pi} \hat{\Pi}^{T} v-\hat{\Pi} \hat{\Pi}^{T} v \hat{\Pi} \hat{\Pi}^{T} u\right) \hat{G}\right\}
$$

Similarly, $\hat{C}_{2}$ is given by

$$
\begin{aligned}
\hat{C}_{2}= & 4 i \frac{\tilde{\alpha}^{2}}{M^{4}} u_{n k} v_{n^{\prime} k^{\prime}}\left[-\left(\hat{\Pi} \hat{\Pi}^{T}\right)^{k l}\left(\hat{\Phi}^{T} \hat{\Phi}\right)_{l m}\left(\hat{\Pi} \hat{\Pi}^{T}\right)^{k^{\prime} l^{\prime}} \hat{\mathbf{G}}_{\left[l^{\prime}\right.}^{[m} \delta_{\left.m^{\prime}\right]}^{n]}\left(\hat{\Pi} \hat{\Pi}^{T}\right)^{m^{\prime} n^{\prime}}\right. \\
& +\left(\hat{\Pi} \hat{\Pi}^{T}\right)^{k l} \hat{\mathbf{G}}_{[l}^{\left[k^{\prime}\right.} \delta_{m]}^{\left.l^{\prime}\right]}\left(\hat{\Phi}^{T} \hat{\Phi}\right)_{l^{\prime} m^{\prime}}\left(\hat{\Pi} \hat{\Pi}^{T}\right)^{m^{\prime} n^{\prime}}\left(\hat{\Pi} \hat{\Pi}^{T}\right)^{m n} \\
& +\left(\hat{\Pi} \hat{\Pi}^{T}\right)^{k l}\left(\hat{\Pi} \hat{\Pi}^{T}\right)^{k^{\prime} l^{\prime}}\left(\hat{\Phi}^{T} \hat{\Phi}\right)_{l^{\prime} m^{\prime}} \hat{\mathbf{G}}_{[l}^{\left[m^{\prime}\right.} \delta_{m]}^{\left.n^{\prime}\right]}\left(\hat{\Pi} \hat{\Pi}^{T}\right)^{m n} \\
& \left.-\left(\hat{\Pi} \hat{\Pi}^{T}\right)^{k^{\prime} l^{\prime}} \hat{\mathbf{G}}_{\left[l^{\prime}\right.}^{[k} \delta_{\left.m^{\prime}\right]}^{l]}\left(\hat{\Pi} \hat{\Pi}^{T}\right)^{m^{\prime} n^{\prime}}\left(\hat{\Phi}^{T} \hat{\Phi}\right)_{l m}\left(\hat{\Pi} \hat{\Pi}^{T}\right)^{m n}\right]
\end{aligned}
$$

In the above expression, the $\operatorname{GL}(L, \mathbb{R})$ generators can be pushed to the far right using the following commutators,

$$
\begin{aligned}
& {\left[\hat{\mathbf{G}}_{[k}^{[i} \delta_{l]}^{j]},\left(\hat{\Pi} \hat{\Pi}^{T}\right)^{m n}\right]=2 i \delta_{[k}^{[i} \delta_{l]}^{<m}\left(\hat{\Pi} \hat{\Pi}^{T}\right)^{j] n>},} \\
& {\left[\hat{\mathbf{G}}_{[k}^{[i} \delta_{l]}^{j]},\left(\hat{\Phi} \hat{\Phi}^{T}\right)_{m n}\right]=-2 i \delta_{[k}^{[i} \delta_{<m}^{j]}\left(\hat{\Phi} \hat{\Phi}^{T}\right)_{l] n>},}
\end{aligned}
$$

where pairs of indices enclosed by [ ] and $<>$ are symmetrized, e.g., $\delta_{[k}^{[i} \delta_{l]}^{<m} A^{j] n>}=$ $\frac{1}{8}\left(\delta_{k}^{i} \delta_{l}^{m} A^{j n}+\delta_{k}^{i} \delta_{l}^{n} A^{j m}+\delta_{l}^{i} \delta_{k}^{m} A^{j n}+\delta_{l}^{i} \delta_{k}^{n} A^{j m}+\delta_{k}^{j} \delta_{l}^{m} A^{i n}+\delta_{k}^{j} \delta_{l}^{n} A^{i m}+\delta_{l}^{j} \delta_{k}^{m} A^{i n}+\delta_{l}^{j} \delta_{k}^{n} A^{i m}\right)$. This allows us to write $\hat{C}_{2}$ as

$$
\hat{C}_{2}=\hat{C}_{21}+\hat{C}_{22}
$$


where

$$
\begin{aligned}
\hat{C}_{21}= & 4 i \frac{\tilde{\alpha}^{2}}{M^{4}} u_{n k} v_{n^{\prime} k^{\prime}}\left[-\left(\hat{\Pi} \hat{\Pi}^{T}\right)^{k l}\left(\hat{\Phi}^{T} \hat{\Phi}\right)_{l i}\left(\hat{\Pi} \hat{\Pi}^{T}\right)^{k^{\prime} i^{\prime}}\left(\hat{\Pi} \hat{\Pi}^{T}\right)^{j^{\prime} n^{\prime}} \delta_{j}^{n}\right. \\
& +\left(\hat{\Pi} \hat{\Pi}^{T}\right)^{k i^{\prime}}\left(\hat{\Phi}^{T} \hat{\Phi}\right)_{j l}\left(\hat{\Pi} \hat{\Pi}^{T}\right)^{l n^{\prime}}\left(\hat{\Pi} \hat{\Pi}^{T}\right)^{j^{\prime} n} \delta_{i}^{k^{\prime}} \\
& +\left(\hat{\Pi} \hat{\Pi}^{T}\right)^{k i^{\prime}}\left(\hat{\Pi} \hat{\Pi}^{T}\right)^{k^{\prime} l}\left(\hat{\Phi}^{T} \hat{\Phi}\right)_{l i}\left(\hat{\Pi} \hat{\Pi}^{T}\right)^{j^{\prime} n} \delta_{j}^{n^{\prime}} \\
& \left.-\left(\hat{\Pi} \hat{\Pi}^{T}\right)^{k^{\prime} i^{\prime}}\left(\hat{\Pi} \hat{\Pi}^{T}\right)^{j^{\prime} n^{\prime}}\left(\hat{\Phi}^{T} \hat{\Phi}\right)_{j l}\left(\hat{\Pi} \hat{\Pi}^{T}\right)^{l n} \delta_{i}^{k}\right] \hat{\mathbf{G}}_{\left[i^{\prime}\right.}^{[i} \delta_{\left.j^{\prime}\right]}^{j]}, \\
\hat{C}_{22}= & \frac{\tilde{\alpha}}{M^{2}}\left[(M-2) \operatorname{tr}\left\{\left(v \hat{\Pi} \hat{\Pi}^{T} u-u \hat{\Pi} \hat{\Pi}^{T} v\right) \hat{H}\right\}\right. \\
& \left.+4\left(\operatorname{tr}\left\{\hat{\Pi} \hat{\Pi}^{T} v\right\} \operatorname{tr}\{\hat{H} u\}-\operatorname{tr}\left\{\hat{\Pi} \hat{\Pi}^{T} u\right\} \operatorname{tr}\{\hat{H} v\}\right)\right] \\
& +4 i \frac{\tilde{\alpha}^{2}}{M^{4}} u_{n k} v_{n^{\prime} k^{\prime}}\left[M\left(\hat{\Pi} \hat{\Pi}^{T}\right)^{k i^{\prime}}\left(\hat{\Pi} \hat{\Pi}^{T}\right)^{j^{\prime} n^{\prime}} \delta_{i}^{k^{\prime}} \delta_{j}^{n}+(M+2)\left(\hat{\Pi} \hat{\Pi}^{T}\right)^{k i^{\prime}}\left(\hat{\Pi} \hat{\Pi}^{T}\right)^{k^{\prime} n} \delta_{i}^{j^{\prime}} \delta_{j}^{n^{\prime}}\right. \\
& +2\left(\hat{\Pi} \hat{\Pi}^{T}\right)^{k i^{\prime}}\left(\hat{\Pi} \hat{\Pi}^{T}\right)^{j^{\prime} n} \delta_{i}^{k^{\prime}} \delta_{j}^{n^{\prime}}-2\left(\hat{\Pi} \hat{\Pi}^{T}\right)^{k n^{\prime}}\left(\hat{\Pi} \hat{\Pi}^{T}\right)^{k^{\prime} i^{\prime}} \delta_{i}^{j^{\prime}} \delta_{j}^{n} \\
& \left.-2\left(\hat{\Pi} \hat{\Pi}^{T}\right)^{k n^{\prime}}\left(\hat{\Pi} \hat{\Pi}^{T}\right)^{j^{\prime} i^{\prime}} \delta_{i}^{k^{\prime}} \delta_{j}^{n}-2\left(\hat{\Pi} \hat{\Pi}^{T}\right)^{n k}\left(\hat{\Pi} \hat{\Pi}^{T}\right)^{n^{\prime} i^{\prime}} \delta_{i}^{j^{\prime}} \delta_{j}^{k^{\prime}}\right] \hat{\mathbf{G}}_{\left[i^{\prime}\right.}^{[i} \delta_{\left.j^{\prime}\right]}^{j]}
\end{aligned}
$$

By writing $\hat{\mathbf{G}}_{\left[i^{\prime}\right.}^{[i} \delta_{\left.j^{\prime}\right]}^{j]}=\hat{G}_{\left[i^{\prime}\right.}^{[i} \delta_{\left.j^{\prime}\right]}^{j]}+\hat{G}_{0} \delta_{\left[i^{\prime}\right.}^{[i} \delta_{\left.j^{\prime}\right]}^{j]}$ following eq. (3.8), the contributions from the $\mathrm{SL}(L, \mathbb{R})$ generators and the global dilatation can be separated. The terms that are proportional to $\hat{G}_{0}$ are canceled between $C_{21}$ and $C_{22}$, and we obtain

$$
\begin{aligned}
\hat{C}_{2}= & 4 i \frac{\tilde{\alpha}^{2}}{M^{4}} u_{n k} v_{n^{\prime} k^{\prime}}\left[-\left(\hat{\Pi} \hat{\Pi}^{T}\right)^{k l}\left(\hat{\Phi}^{T} \hat{\Phi}\right)_{l i}\left(\hat{\Pi} \hat{\Pi}^{T}\right)^{k^{\prime} i^{\prime}}\left(\hat{\Pi} \hat{\Pi}^{T}\right)^{j^{\prime} n^{\prime}} \delta_{j}^{n}\right. \\
& +\left(\hat{\Pi} \hat{\Pi}^{T}\right)^{k i^{\prime}}\left(\hat{\Phi}^{T} \hat{\Phi}\right)_{j l}\left(\hat{\Pi} \hat{\Pi}^{T}\right)^{l n^{\prime}}\left(\hat{\Pi} \hat{\Pi}^{T}\right)^{j^{\prime} n} \delta_{i}^{k^{\prime}} \\
& +\left(\hat{\Pi} \hat{\Pi}^{T}\right)^{k i^{\prime}}\left(\hat{\Pi} \hat{\Pi}^{T}\right)^{k^{\prime} l}\left(\hat{\Phi}^{T} \hat{\Phi}\right)_{l i}\left(\hat{\Pi} \hat{\Pi}^{T}\right)^{j^{\prime} n} \delta_{j}^{n^{\prime}} \\
& -\left(\hat{\Pi} \hat{\Pi}^{T}\right)^{k^{\prime} i^{\prime}}\left(\hat{\Pi} \hat{\Pi}^{T}\right)^{j^{\prime} n^{\prime}}\left(\hat{\Phi}^{T} \hat{\Phi}\right)_{j l}\left(\hat{\Pi} \hat{\Pi}^{T}\right)^{l n} \delta_{i}^{k} \\
& +M\left(\hat{\Pi} \hat{\Pi}^{T}\right)^{k i^{\prime}}\left(\hat{\Pi} \hat{\Pi}^{T}\right)^{j^{\prime} n^{\prime}} \delta_{i}^{k^{\prime}} \delta_{j}^{n}+(M+2)\left(\hat{\Pi} \hat{\Pi}^{T}\right)^{k i^{\prime}}\left(\hat{\Pi} \hat{\Pi}^{T}\right)^{k^{\prime} n} \delta_{i}^{j^{\prime}} \delta_{j}^{n^{\prime}} \\
& +2\left(\hat{\Pi} \hat{\Pi}^{T}\right)^{k i^{\prime}}\left(\hat{\Pi} \hat{\Pi}^{T}\right)^{j^{\prime} n} \delta_{i}^{k^{\prime}} \delta_{j}^{n^{\prime}}-2\left(\hat{\Pi} \hat{\Pi}^{T}\right)^{k n^{\prime}}\left(\hat{\Pi} \hat{\Pi}^{T}\right)^{k^{\prime} i^{\prime}} \delta_{i}^{j^{\prime}} \delta_{j}^{n} \\
& \left.\left.-2\left(\hat{\Pi} \hat{\Pi}^{T}\right)^{k n^{\prime}}\left(\hat{\Pi} \hat{\Pi}^{T}\right)^{j^{\prime} i^{\prime}} \delta_{i}^{k^{\prime}} \delta_{j}^{n}-2\left(\hat{\Pi} \hat{\Pi}^{T}\right)^{n k}\left(\hat{\Pi} \hat{\Pi}^{T}\right)^{n^{\prime} i^{\prime}} \delta_{i}^{j^{\prime}} \delta_{j}^{k^{\prime}}\right] \hat{G}_{\left[i^{\prime}\right.}^{[i} j_{\left.j^{\prime}\right]}\right] \\
& +\frac{\tilde{\alpha}}{M}\left[(M-2) \operatorname{tr}\left\{\left(v \hat{\Pi} \hat{\Pi}^{T} u-u \hat{\Pi} \hat{\Pi}^{T} v\right) \hat{H}\right\}+4\left(\operatorname{tr}\left\{\hat{\Pi} \hat{\Pi}^{T} v\right\} \operatorname{tr}\{\hat{H} u\}\right.\right. \\
& \left.\left.-\operatorname{tr}\left\{\hat{\Pi} \hat{\Pi}^{T} u\right\} \operatorname{tr}\{\hat{H} v\}\right)\right] .
\end{aligned}
$$

Combining eq. (C.9) with eq. (C.4), we obtain eq. (3.27). 


\section{Poisson brackets of the momentum and Hamiltonian}

From eq. (4.16), the Poisson bracket between momentum constraints is given by

$$
\begin{aligned}
\left\{\mathscr{G}_{x}, \mathscr{G}_{y}\right\}_{\mathrm{PB}} & =\left\{\operatorname{tr}\left\{\left(s q+2 t_{c} p_{c}\right) x\right\}, \operatorname{tr}\left\{\left(s q+2 t_{c^{\prime}} p_{c^{\prime}}\right) y\right\}\right\}_{\mathrm{PB}} \\
& =\operatorname{tr}\left\{x s q y-q x y s+2\left(x t_{c} p_{c} y+x t_{c} y^{T} p_{c}\right)-2\left(p_{c} x y t_{c}+p_{c} x t_{c} y^{T}\right)\right\} \\
& =\mathscr{G}_{y x-x y} .
\end{aligned}
$$

By choosing $x_{i^{\prime}}^{j^{\prime}}=\delta_{j}^{j^{\prime}} \delta_{i^{\prime}}^{i}, y_{k^{\prime}}^{l^{\prime}}=\delta_{l}^{l^{\prime}} \delta_{k^{\prime}}^{k}$, we obtain eq. (4.17).

To prove eq. (4.18), we first note

$$
\begin{aligned}
& \left\{\mathscr{G}_{x}, \operatorname{tr}\{U v\}\right\}_{\mathrm{PB}}=U_{v x+x^{T} v}, \\
& \left\{\mathscr{G}_{x}, \operatorname{tr}\{Q \tilde{v}\}\right\}_{\mathrm{PB}}=-Q_{x \tilde{v}+\tilde{v} x^{T}} .
\end{aligned}
$$

Here $U=\left(s s^{T}+\sum_{c}\left[4 t_{c} p_{c} t_{c}-i t_{c}\right]\right)$ and $Q=\left(q^{T} q+\sum_{c} p_{c}\right) . v$ and $\tilde{v}$ are covariant and contravariant symmetric tensors that are independent of $s, q, t_{c}, p_{c}$, respectively. Using the distribution rule, we write the Poisson bracket between the momentum and the Hamiltonian constraints as

$$
\begin{aligned}
\left\{\mathscr{G}_{x}, \mathscr{H}_{v}\right\}_{\mathrm{PB}} & =\left\{\mathscr{G}_{x},(-U+\tilde{\alpha} U Q U)_{v}\right\}_{\mathrm{PB}} \\
& =-\left\{\mathscr{G}_{x}, U_{v}\right\}_{\mathrm{PB}}+\tilde{\alpha}\left\{\mathscr{G}_{x}, U_{Q U v}\right\}_{\mathrm{PB}}+\tilde{\alpha}\left\{\mathscr{G}_{x}, Q_{U v U}\right\}_{\mathrm{PB}}+\tilde{\alpha}\left\{\mathscr{G}_{x}, U_{v U Q}\right\}_{\mathrm{PB}} .
\end{aligned}
$$

Here $\left\{\mathscr{G}_{x}, U_{A}\right\}_{\mathrm{PB}} \equiv\left\{\mathscr{G}^{i}{ }_{j}, U^{l m}\right\}_{\mathrm{PB}} x_{i}{ }_{i} A_{m l}$ and $\left\{\mathscr{G}_{x}, Q_{B}\right\}_{\mathrm{PB}} \equiv\left\{\mathscr{G}^{i}{ }_{j}, Q_{l m}\right\}_{\mathrm{PB}} x_{i}{ }_{i} B^{m l}$. $A$ and $B$ are covariant and contravariant tensors that are made of $v, x, s, q, t, p$. From eq. (D.2), we obtain

$$
\left\{\mathscr{G}_{x}, \mathscr{H}_{v}\right\}_{\mathrm{PB}}=(-U+\tilde{\alpha} U Q U)_{v x+x^{T} v} .
$$

In order to compute the Poisson bracket between Hamiltonians, we use

$$
\begin{aligned}
& \left\{\operatorname{tr}\left\{U v_{1}\right\}, \operatorname{tr}\left\{U v_{2}\right\}\right\}_{\mathrm{PB}}=0, \\
& \left\{\operatorname{tr}\left\{Q v_{1}\right\}, \operatorname{tr}\left\{Q v_{2}\right\}\right\}_{\mathrm{PB}}=0, \\
& \left\{\operatorname{tr}\left\{U v_{1}\right\}, \operatorname{tr}\left\{Q v_{2}\right\}\right\}_{\mathrm{PB}}=-4 \mathscr{G}_{v_{2} v_{1}} .
\end{aligned}
$$

Similar to eq. (D.3), we write the Poisson bracket between Hamiltonians as

$$
\begin{aligned}
\left\{\mathscr{H}_{v_{1}}, \mathscr{H}_{v_{2}}\right\}_{\mathrm{PB}}= & \left\{\operatorname{tr}\left\{(-U+\tilde{\alpha} U Q U) v_{1}\right\}, \operatorname{tr}\left\{(-U+\tilde{\alpha} U Q U) v_{2}\right\}\right\}_{\mathrm{PB}} \\
= & \tilde{\alpha}\left[-\left\{U_{v_{1}}, Q_{U v_{2} U}\right\}_{\mathrm{PB}}+\left\{U_{v_{2}}, Q_{U v_{1} U}\right\}_{\mathrm{PB}}\right] \\
& +\tilde{\alpha}^{2}\left[\left\{U_{Q U v_{1}}, Q_{U v_{2} U}\right\}_{\mathrm{PB}}+\left\{Q_{U v_{1} U}, U_{Q U v_{2}}\right\}_{\mathrm{PB}}\right. \\
& \left.+\left\{Q_{U v_{1} U}, U_{v_{2} U Q}\right\}_{\mathrm{PB}}+\left\{U_{v_{1} U Q}, Q_{U v_{2} U}\right\}_{\mathrm{PB}}\right] .
\end{aligned}
$$


From eq. (D.5), we obtain

$$
\begin{aligned}
\left\{\mathscr{H}_{v_{1}}, \mathscr{H}_{v_{2}}\right\}_{\mathrm{PB}}= & -4 \operatorname{tr}\left\{\mathscr { G } \left[-\tilde{\alpha}\left(U v_{2} U v_{1}-U v_{1} U v_{2}\right)\right.\right. \\
& \left.\left.+\tilde{\alpha}^{2}\left(U v_{2} U Q U v_{1}+U v_{2} U v_{1} U Q-U v_{1} U Q U v_{2}-U v_{1} U v_{2} U Q\right)\right]\right\} .
\end{aligned}
$$

By choosing $\left(v_{1}\right)_{i^{\prime} j^{\prime}}=\delta_{i^{\prime} j^{\prime}}^{i j},\left(v_{2}\right)_{l^{\prime} k^{\prime}}=\delta_{l^{\prime} k^{\prime}}^{l k}$, we obtain eq. (4.19).

\section{E Derivations of the transformed shift and lapse functions}

In this appendix, we derive eqs. (5.6), (5.7), (5.15) and (5.16).

\section{E.1 Eq. (5.6)}

From eq. (3.14), the scalar field associated with the shift tensor $y x-x y$ is given by

$$
\zeta_{y x-x y}\left(r_{i}\right)=\sum_{j, k}\left(y_{k}^{j} x_{i}^{k}-x_{k}^{j} y_{i}^{k}\right)
$$

The sum over $j$ gives

$$
\zeta_{y x-x y}\left(r_{i}\right)=\sum_{k}\left(\zeta_{y}\left(r_{k}\right) x_{i}^{k}-\zeta_{x}\left(r_{k}\right) y_{i}^{k}\right)
$$

Now, we expand $\zeta_{y}\left(r_{k}\right)$ and $\zeta_{x}\left(r_{k}\right)$ around $r_{i}$ to write

$$
\begin{aligned}
\zeta_{y x-x y}\left(r_{i}\right)= & \sum_{k}\left(\left[\zeta_{y}\left(r_{i}\right)+\partial_{\mu} \zeta_{y}\left(r_{i}\right)\left(r_{k}^{\mu}-r_{i}^{\mu}\right)+O\left(\partial^{2}\right)\right] x_{i}^{k}\right. \\
& \left.-\left[\zeta_{x}\left(r_{i}\right)+\partial_{\mu} \zeta_{x}\left(r_{i}\right)\left(r_{k}^{\mu}-r_{i}^{\mu}\right)+O\left(\partial^{2}\right)\right] y^{k}\right) \\
= & \xi_{x}^{\mu}\left(r_{i}\right) \partial_{\mu} \zeta_{y}\left(r_{i}\right)-\xi_{y}^{\mu}\left(r_{i}\right) \partial_{\mu} \zeta_{x}\left(r_{i}\right)+O\left(\partial^{2}\right) .
\end{aligned}
$$

E.2 Eq. (5.7)

From eq. (3.15), the vector field associated with the shift tensor $y x-x y$ is given by

$$
\xi_{y x-x y}^{\mu}\left(r_{i}\right)=\sum_{j, k}\left(y_{k}^{j} x_{i}^{k}-x_{k}^{j} y_{i}^{k}\right)\left(r_{j}^{\mu}-r_{i}^{\mu}\right) .
$$

Expanding $y^{j}{ }_{k}$ and $x_{k}^{j}$ around $r_{k}=r_{i}$ gives

$$
\begin{aligned}
\xi_{y x-x y}^{\mu}\left(r_{i}\right)= & \sum_{j, k}\left(\left[y_{i}^{j}+\frac{\partial y^{j}}{\partial r_{i}^{\nu}}\left(r_{k}^{\nu}-r_{i}^{\nu}\right)+O\left(\partial^{2}\right)\right] x^{k}{ }_{i}\right. \\
& \left.-\left[x^{j}{ }_{i}+\frac{\partial x^{j}{ }_{i}}{\partial r_{i}^{\nu}}\left(r_{k}^{\nu}-r_{i}^{\nu}\right)+O\left(\partial^{2}\right)\right] y_{i}^{k}\right)\left(r_{j}^{\mu}-r_{i}^{\mu}\right) \\
= & \xi_{y}^{\mu}\left(r_{i}\right) \zeta_{x}\left(r_{i}\right)+\xi_{x}^{\nu}\left(r_{i}\right)\left(\frac{\partial \xi_{y}^{\mu}\left(r_{i}\right)}{\partial r_{i}^{\nu}}+\delta_{\nu}^{\mu} \zeta_{y}\left(r_{i}\right)\right) \\
& -\xi_{x}^{\mu}\left(r_{i}\right) \zeta_{y}\left(r_{i}\right)-\xi_{y}^{\nu}\left(r_{i}\right)\left(\frac{\partial \xi_{x}^{\mu}\left(r_{i}\right)}{\partial r_{i}^{\nu}}+\delta_{\nu}^{\mu} \zeta_{x}\left(r_{i}\right)\right)+O\left(\partial^{2}\right) \\
= & \xi_{x}^{\nu}\left(r_{i}\right) \partial_{\nu} \xi_{y}^{\mu}\left(r_{i}\right)-\xi_{y}^{\nu}\left(r_{i}\right) \partial_{\nu} \xi_{x}^{\mu}\left(r_{i}\right)+O\left(\partial^{2}\right) .
\end{aligned}
$$


E.3 Eq. (5.15)

From eq. (5.11), the lapse function associated with the shift tensor $v x+x^{T} v$ is given by

$$
\begin{aligned}
\theta_{v x+x^{T} v}\left(r_{i}\right) & =2 \sum_{k} v_{i k} x_{i}^{k}, \\
\lambda_{v x+x^{T} v}\left(r_{i}, r_{j}\right) & =\sum_{k}\left(v_{i k} x^{k}{ }_{j}+v_{j k} x_{i}^{k}\right) .
\end{aligned}
$$

Since $\theta\left(r_{i}\right)$ can be obtained from $\lambda\left(r_{i}, r_{j}\right)$ by setting $r_{j}=r_{i}$, we first consider the latter. Expanding $v_{i k}$ around $r_{k}=r_{j}$, and $v_{j k}$ around $r_{k}=r_{i}$ gives

$$
\begin{aligned}
\lambda_{v x+x^{T} v}\left(r_{i}, r_{j}\right) & =\sum_{k}\left(\left[v_{i j}+\frac{\partial v_{i j}}{\partial r_{j}^{\mu}}\left(r_{k}^{\mu}-r_{j}^{\mu}\right)+O\left(\partial^{2}\right)\right] x^{k}{ }_{j}+\left[v_{j i}+\frac{\partial v_{j i}}{\partial r_{i}^{\mu}}\left(r_{k}^{\mu}-r_{i}^{\mu}\right)+O\left(\partial^{2}\right)\right] x_{i}^{k}\right) \\
& =v_{i j}\left[\zeta_{x}\left(r_{j}\right)+\zeta_{x}\left(r_{i}\right)\right]+\frac{\partial v_{i j}}{\partial r_{j}^{\mu}} \xi_{x}^{\mu}\left(r_{j}\right)+\frac{\partial v_{i j}}{\partial r_{i}^{\mu}} \xi_{x}^{\mu}\left(r_{i}\right)+O\left(\partial^{2}\right) \\
& =\left[\zeta_{x}\left(r_{i}\right)+\zeta_{x}\left(r_{j}\right)\right] \lambda_{v}\left(r_{i}, r_{j}\right)+\xi_{x}^{\mu}\left(r_{j}\right) \frac{\partial \lambda_{v}\left(r_{i}, r_{j}\right)}{\partial r_{j}^{\mu}}+\xi_{x}^{\mu}\left(r_{i}\right) \frac{\partial \lambda_{v}\left(r_{i}, r_{j}\right)}{\partial r_{i}^{\mu}}+O\left(\partial^{2}\right) .
\end{aligned}
$$

For $\theta_{v x+x^{T} v}$, we can simply set $r_{j}=r_{i}$ in eq. (E.7),

$$
\theta_{v x+x^{T} v}\left(r_{i}\right)=2 \zeta_{x}\left(r_{i}\right) \theta_{v}\left(r_{i}\right)+\xi_{x}^{\mu}\left(r_{i}\right) \frac{\partial \theta_{v}\left(r_{i}\right)}{\partial r_{i}^{\mu}}
$$

\section{E.4 Eq. (5.16)}

From eq. (4.19), we have

$$
\left\{H_{u}, H_{v}\right\}_{\mathrm{PB}}=\mathscr{G}_{n}^{m} \mathscr{C}_{m}^{i j k l n} u_{j i} v_{l k}
$$

where $\mathscr{C}_{m}^{i j k l n}$ is given by eq. (4.20). For diagonal $u$ and $v$, we write

$$
\left\{H_{u}, H_{v}\right\}_{\mathrm{PB}}=\mathscr{G}_{n}^{m} \mathscr{C}_{m}^{r_{1} r_{1} r_{2} r_{2} n} \theta_{u}\left(r_{1}\right) \theta_{v}\left(r_{2}\right)+O\left(\partial^{2}\right),
$$

where eq. (5.11) is used. Now we expand $\theta_{u}\left(r_{1}\right)$ and $\theta_{v}\left(r_{2}\right)$ near $r_{1}=r_{m}$ and $r_{2}=r_{m}$ to write

$$
\begin{aligned}
& \left\{H_{u}, H_{v}\right\}_{\mathrm{PB}}=\mathscr{G}_{n}^{m} \mathscr{C}_{m}^{r_{1} r_{1} r_{2} r_{2} n}\left[\theta_{u}\left(r_{m}\right)+\nabla_{\mu} \theta_{u}\left(r_{m}\right)\left(r_{1}^{\mu}-r_{m}^{\mu}\right)\right]\left[\theta_{v}\left(r_{m}\right)+\nabla_{\nu} \theta_{v}\left(r_{m}\right)\left(r_{2}^{\nu}-r_{m}^{\nu}\right)\right] \\
& +O\left(\partial^{2}\right) \text {. }
\end{aligned}
$$

Because $\mathscr{C}_{m}^{r_{1} r_{1} r_{2} r_{2} n}=-\mathscr{C}_{m}^{r_{2} r_{2} r_{1} r_{1} n}$, eq. (E.11) can be written as

$$
\begin{aligned}
\left\{H_{u}, H_{v}\right\}_{\mathrm{PB}}= & \frac{1}{2} \mathscr{G}_{n}^{m} \mathscr{C}_{m}^{r_{1} r_{1} r_{2} r_{2} n}\left\{\left[\theta_{u}\left(r_{m}\right)+\nabla_{\mu} \theta_{u}\left(r_{m}\right)\left(r_{1}^{\mu}-r_{m}^{\mu}\right)\right]\left[\theta_{v}\left(r_{m}\right)+\nabla_{\nu} \theta_{v}\left(r_{m}\right)\left(r_{2}^{\nu}-r_{m}^{\nu}\right)\right]\right. \\
& \left.-\left[\theta_{u}\left(r_{m}\right)+\nabla_{\mu} \theta_{u}\left(r_{m}\right)\left(r_{2}^{\mu}-r_{m}^{\mu}\right)\right]\left[\theta_{v}\left(r_{m}\right)+\nabla_{\nu} \theta_{v}\left(r_{m}\right)\left(r_{1}^{\nu}-r_{m}^{\nu}\right)\right]\right\}+O\left(\partial^{2}\right) .
\end{aligned}
$$


To the leading order in the derivative expansion, we obtain

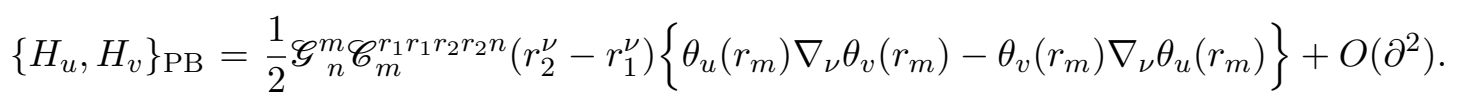

By using eq. (5.1) and eq. (5.2), we obtain eq. (5.16).

\section{F Metric in translationally invariant states}

We first note that $C_{m}^{i j k l n}$ in eq. (4.20) reduces to

$$
C_{m}^{i j k l n}=-4 \tilde{\alpha}\left(U^{n[l} U^{k][j} \delta_{m}^{i]}-U^{n[j} U^{i][l} \delta_{m}^{k]}\right)
$$

on the constraint hypersurface that satisfy $\tilde{\alpha} Q U=\tilde{\alpha} U Q=I$. Inserting eq. (F.1) to eq. (5.18) and eq. (5.19), we write

$$
\begin{aligned}
-\mathcal{S} g^{\mu \nu} & =\frac{1}{4} \sum_{i, l, n} C_{m}^{i i l l n}\left(r_{n m}^{\mu} r_{l i}^{\nu}+r_{n m}^{\nu} r_{l i}^{\mu}\right) \\
& =-2 \tilde{\alpha} \sum_{l, n} U^{n l} U^{l m}\left(r_{n m}^{\mu} r_{l m}^{\nu}+r_{n m}^{\nu} r_{l m}^{\mu}\right),
\end{aligned}
$$

where $r_{n m}^{\mu}=r_{n}^{\mu}-r_{m}^{\mu}$. In the presence of the translational symmetry, we write

$$
U^{n l}=\frac{1}{L} \sum_{\mathbf{k}} e^{i \mathbf{k}\left(\mathbf{r}_{n}-\mathbf{r}_{l}\right)} U_{\mathbf{k}}
$$

to rewrite the metric as

$$
-\mathcal{S} g^{\mu \nu}=-2 \tilde{\alpha} \sum_{l, n} \int \frac{d \mathbf{k} d \mathbf{k}^{\prime}}{(2 \pi)^{2 D}} U_{\mathbf{k}} U_{\mathbf{k}^{\prime}}\left[-\left(\frac{\partial}{\partial k_{\mu}}+\frac{\partial}{\partial k_{\mu}^{\prime}}\right) \frac{\partial}{\partial k_{\nu}^{\prime}}-\left(\frac{\partial}{\partial k_{\nu}}+\frac{\partial}{\partial k_{\nu}^{\prime}}\right) \frac{\partial}{\partial k_{\mu}^{\prime}}\right] e^{i \mathbf{k r}_{n l}+i \mathbf{k}^{\prime} \mathbf{r}_{l m}} .
$$

Integrating $\mathbf{k}, \mathbf{k}^{\prime}$ by part followed by the sum over $r_{l}, r_{n}$ results in

$$
-\mathcal{S} g^{\mu \nu}=4 \tilde{\alpha}\left(\frac{\partial U_{\mathbf{k}}}{\partial k_{\mu}} \frac{\partial U_{\mathbf{k}}}{\partial k_{\nu}}+U_{\mathbf{k}} \frac{\partial^{2} U_{\mathbf{k}}}{\partial k_{\mu} \partial k_{\nu}}\right)_{\mathbf{k}=0} .
$$

\section{G Transformation of the collective variables under boost}

In this appendix, we derive eq. (7.35).

\section{G.1 The shift vector}

From eq. (4.22), the shift tensor is transformed as

$$
\begin{aligned}
\delta y^{n}{ }_{m} & =\partial_{\tau} \eta_{m}^{n}+\eta_{i}^{j} y_{k}^{l} \mathscr{A}_{j l m}^{i k n}+\rho_{j i} v_{l k} \mathscr{C}_{m}^{i j k l n} \\
& =\left(\partial_{\tau} \eta+y \eta-\eta y\right)_{m}^{n}+\rho_{i i} v_{k k} \mathscr{C}_{m}^{i i k k n} .
\end{aligned}
$$


The change in the shift vector is given by

$$
\delta \xi^{\mu}\left(r_{m}\right)=\sum_{n} \delta y_{m}^{n}\left(r_{n}^{\mu}-r_{m}^{\mu}\right) .
$$

Since $y$ in eq. (6.14) is symmetric and $\eta$ is anti-symmetric, $(y \eta-\eta y)$ is a symmetric matrix. Furthermore, $(y \eta-\eta y)_{m}^{n}$ depends on $n$ and $m$ only through $r_{n}-r_{m}$ due to the spatial translational invariance of $y$ and $\eta$ in eq. (6.14) and eq. (7.32). As a result,

$$
\begin{aligned}
(*) & \equiv \sum_{n}(y \eta-\eta y)_{m}^{n}\left(r_{n}^{\mu}-r_{m}^{\mu}\right)=\sum_{n}(y \eta-\eta y)_{0}^{n} r_{n}^{\mu} \\
& =\sum_{n}(y \eta-\eta y)_{0}^{-n} r_{-n}^{\mu}=\sum_{n}(y \eta-\eta y)_{n}^{0} r_{-n}^{\mu} \\
& =-\sum_{n}(y \eta-\eta y)_{0}^{n} r_{n}^{\mu}=-(*) .
\end{aligned}
$$

Here we choose the label of sites such that $r_{-n}=-r_{n}$, and use the fact that $(y \eta-\eta y)^{n}{ }_{m}=$ $(y \eta-\eta y)_{n}^{m}=(y \eta-\eta y)_{m+k}^{n+k}$ for any $k$. This shows $(*)=0$, and

$$
\begin{aligned}
\delta \xi^{\mu}\left(r_{m}\right) & =\partial_{\tau} \sum_{n} \eta_{m}^{n}\left(r_{n}^{\mu}-r_{m}^{\mu}\right)-4 \tilde{\alpha} \sum_{n, i}\left[U^{n i} U^{i m} \rho_{m m}-U^{n i} \rho_{i i} U^{i m}\right]\left(r_{n}^{\mu}-r_{m}^{\mu}\right) \\
& =\left[\varepsilon \zeta^{\mu}+4 \tilde{\alpha} \sum_{n, i} U^{n i} \rho_{i i} U^{i m}\left(r_{n}^{\mu}-r_{m}^{\mu}\right)\right] \\
& =\varepsilon[1+\mathcal{S}] \zeta^{\mu} .
\end{aligned}
$$

In the first and the second lines, we use eq. (F.1) and $\sum_{n, i} U^{n i} U^{i m} \rho_{m m}\left(r_{n}^{\mu}-r_{m}^{\mu}\right)=0$ which follows from the facts that $\sum_{i} U^{n i} U^{i m}$ is symmetric under the exchange of $n$ and $m$, and depends only on $r_{n}-r_{m}$ due to the translational invariance of the saddle point solution. From the second line to the third line, we use $\sum_{n, i} U^{n i} \rho_{i i} U^{i m}\left(r_{n}^{\mu}-r_{m}^{\mu}\right)=\varepsilon \zeta_{\nu} \sum_{n, i} U^{n i} U^{i m} r_{i}^{\nu}\left(r_{n}^{\mu}-\right.$ $\left.r_{m}^{\mu}\right)=\varepsilon \zeta_{\nu} \sum_{n, i} U^{n i} U^{i m}\left(r_{i}^{\nu}-r_{m}^{\nu}+r_{m}^{\nu}\right)\left(r_{n}^{\mu}-r_{m}^{\mu}\right)=\varepsilon \zeta_{\nu} \sum_{n, i} U^{n i} U^{i m}\left(r_{i}^{\nu}-r_{m}^{\nu}\right)\left(r_{n}^{\mu}-r_{m}^{\mu}\right)=$ $\varepsilon \frac{\mathcal{S}}{4 \tilde{\alpha}} g^{\nu \mu} \zeta_{\nu}$ which follows from eq. (F.2). Eq. (G.4) vanishes when the spacetime has the Lorentzian signature, $\delta=-1$.

\section{G.2 The lapse function}

Under the boost, the diagonal component of the lapse tensor is transformed as

$$
\begin{aligned}
\delta v_{n n} & =\partial_{\tau} \rho_{n n}+\eta_{i}^{j} v_{l k} \mathscr{B}_{j n n}^{i k l}-\rho_{k l} y_{i}^{j} \mathscr{B}_{j n n}^{i k l} \\
& =2\left(\eta_{n}^{n} v_{n n}-y_{n}^{n} \rho_{n n}\right)=0 .
\end{aligned}
$$

Here $n$ is not summed over. This vanishes because $\eta$ in eq. (7.32) is anti-symmetric, and $y_{n}^{n}=0$ for the translationally invariant saddle-point solution in eq. (6.14). Therefore, $\delta \theta(r)=0$.

\section{G.3 $p_{c}$}

Under the boost, $p_{c}$ transforms as

$$
\begin{aligned}
\delta p_{c} & =\left\{p_{c}, \mathscr{G}_{\eta}\right\}_{\mathrm{PB}}+\left\{p_{c}, \mathscr{H}_{\rho}\right\}_{\mathrm{PB}} \\
& =\left(p_{c} \eta+\eta^{T} p_{c}\right)+\left(4 p_{c} t_{c} \rho+4 \rho t_{c} p_{c}-i \rho\right) \\
& =4\left(p_{c} \tilde{t}_{c} \rho+\rho \tilde{t}_{c} p_{c}\right) .
\end{aligned}
$$


The first term in the second line vanishes because $\left(p_{c} \eta+\eta^{T} p_{c}\right)_{i j}=p_{c, i l} \eta_{j}^{l}+p_{c, j l} \eta_{i}^{l}=$ $p_{c, i l} \eta_{j}^{l}+p_{c, j(j+i-l)} \eta_{i}^{j+i-l}=p_{c, i l} \eta_{j}^{l}+p_{c, l i} \eta_{l}^{j}=p_{c, i l} \eta_{j}^{l}-p_{c, i l} \eta_{j}^{l}=0$, where we use the fact that $p_{c, i l}=p_{c, l i}=p_{c,(i+k)(l+k)}$ and $\eta_{j}^{i}=-\eta_{i}^{j}=\eta_{j+k}^{i+k}$ for any $k$. From the second line to the third line, we use eq. (6.22).

\section{G.4 $q$}

Under the boost generated by eq. (7.31), $q$ transforms as

$$
\begin{aligned}
\delta q & =\left\{q, \mathscr{G}_{\eta}\right\}_{\mathrm{PB}}+\left\{q, \mathscr{H}_{\rho}\right\}_{\mathrm{PB}}+\left\{q, \mathscr{T}_{\tilde{o}_{L}}\right\}_{\mathrm{PB}} \\
& =\left(\bar{q} \eta+2 s^{T} \rho+\tilde{o}_{L} \bar{q}\right),
\end{aligned}
$$

Since $\eta$ is anti-symmetric and $\bar{q}=q_{d} I$, the transformation generated by $\mathscr{G}_{\eta}$ can be canceled by a $\mathrm{O}(L)$ flavour rotation. By choosing $\tilde{o}_{L}=-\eta$, we obtain

$$
\delta q=2 s^{T} \rho .
$$

Open Access. This article is distributed under the terms of the Creative Commons Attribution License (CC-BY 4.0), which permits any use, distribution and reproduction in any medium, provided the original author(s) and source are credited.

\section{References}

[1] A. Einstein, Die Grundlage der allgemeinen Relativitätstheorie, Ann. Phys. 49 (1916) 769.

[2] B.S. DeWitt, Quantum Theory of Gravity. 1. The Canonical Theory, Phys. Rev. 160 (1967) 1113 [INSPIRE].

[3] T. Regge, General relativity without coordinates, Nuovo Cim. 19 (1961) 558.

[4] S. Weinberg, Ultraviolet divergences in quantum theories of gravitation, in General Relativity: An Einstein Centenary Survey, Cambridge University Press, Cambridge U.K. (1980), pg. 790.

[5] A. Ashtekar, New Variables for Classical and Quantum Gravity, Phys. Rev. Lett. 57 (1986) 2244 [INSPIRE].

[6] P. Horava, Quantum Gravity at a Lifshitz Point, Phys. Rev. D 79 (2009) 084008 [arXiv: 0901.3775] [INSPIRE].

[7] P. Di Francesco, P.H. Ginsparg and J. Zinn-Justin, 2 - D Gravity and random matrices, Phys. Rept. 254 (1995) 1 [hep-th/9306153] [INSPIRE].

[8] J.M. Maldacena, The Large $N$ limit of superconformal field theories and supergravity, Int. J. Theor. Phys. 38 (1999) 1113 [hep-th/9711200] [INSPIRE].

[9] E. Witten, Anti-de Sitter space and holography, Adv. Theor. Math. Phys. 2 (1998) 253 [hep-th/9802150] [INSPIRE].

[10] S.S. Gubser, I.R. Klebanov and A.M. Polyakov, Gauge theory correlators from noncritical string theory, Phys. Lett. B 428 (1998) 105 [hep-th/9802109] [INSPIRE].

[11] T. Banks, W. Fischler, S.H. Shenker and L. Susskind, $M$ theory as a matrix model: A Conjecture, Phys. Rev. D 55 (1997) 5112 [hep-th/9610043] [INSPIRE]. 
[12] J. Polchinski, String Theory, Cambridge Monographs on Mathematical Physics, Cambridge University Press, Cambridge U.K. (1998).

[13] D. Oriti, The microscopic dynamics of quantum space as a group field theory, in Proceedings of Foundations of Space and Time: Reflections on Quantum Gravity Cape Town South Africa (2011), pg. 257 [arXiv:1110.5606] [INSPIRE].

[14] C. Rovelli and F. Vidotto, Covariant Loop Quantum Gravity, Cambridge Monographs on Mathematical Physics, Cambridge University Press, Cambridge U.K. (2014) [ISBN:9781107069626].

[15] S. Carlip, Quantum gravity: A Progress report, Rept. Prog. Phys. 64 (2001) 885 [gr-qc/0108040] [INSPIRE].

[16] H. Hamber, Quantum Gravitation: The Feynman Path Integral Approach, Springer, Berlin Germany (2009) [ISBN:978-3-540-85292-6].

[17] R. Loll, Quantum Gravity from Causal Dynamical Triangulations: A Review, Class. Quant. Grav. 37 (2020) 013002 [arXiv: 1905.08669] [INSPIRE].

[18] G. 't Hooft and M. Veltman, One-loop divergencies in the theory of gravitation, Ann. Henri Poincaré A 20 (1974) 69.

[19] M.H. Goroff and A. Sagnotti, The Ultraviolet Behavior of Einstein Gravity, Nucl. Phys. B 266 (1986) 709 [INSPIRE].

[20] N. Seiberg, Emergent spacetime, in The Quantum Structure of Space and Time: Proceedings of the 23rd Solvay Conference on Physics, Brussels Belgium (2005), pg. 163 [hep-th/0601234] [INSPIRE].

[21] E.H. Wichmann and J.H. Crichton, Cluster Decomposition Properties of the S Matrix, Phys. Rev. 132 (1963) 2788 [INSPIRE].

[22] D. Marolf, Emergent Gravity Requires Kinematic Nonlocality, Phys. Rev. Lett. 114 (2015) 031104 [arXiv: 1409.2509] [INSPIRE].

[23] J.F. Donoghue, Introduction to the effective field theory description of gravity, in Advanced School on Effective Theories, Almunecar Spain (1995) [gr-qc/9512024] [INSPIRE].

[24] C.P. Burgess, Quantum gravity in everyday life: General relativity as an effective field theory, Living Rev. Rel. 7 (2004) 5 [gr-qc/0311082] [INSPIRE].

[25] S.-S. Lee, Emergent gravity from relatively local Hamiltonians and a possible resolution of the black hole information puzzle, JHEP 10 (2018) 043 [arXiv:1803.00556] [INSPIRE].

[26] S. Ryu and T. Takayanagi, Holographic derivation of entanglement entropy from AdS/CFT, Phys. Rev. Lett. 96 (2006) 181602 [hep-th/0603001] [INSPIRE].

[27] V.E. Hubeny, M. Rangamani and T. Takayanagi, A Covariant holographic entanglement entropy proposal, JHEP 07 (2007) 062 [arXiv:0705.0016] [INSPIRE].

[28] M. Van Raamsdonk, Building up spacetime with quantum entanglement, Gen. Rel. Grav. 42 (2010) 2323 [arXiv: 1005.3035] [INSPIRE].

[29] H. Casini, M. Huerta and R.C. Myers, Towards a derivation of holographic entanglement entropy, JHEP 05 (2011) 036 [arXiv:1102.0440] [INSPIRE].

[30] A. Lewkowycz and J. Maldacena, Generalized gravitational entropy, JHEP 08 (2013) 090 [arXiv: 1304.4926] [INSPIRE]. 
[31] J. Maldacena and L. Susskind, Cool horizons for entangled black holes, Fortsch. Phys. 61 (2013) 781 [arXiv:1306.0533] [INSPIRE].

[32] T. Jacobson, Thermodynamics of space-time: The Einstein equation of state, Phys. Rev. Lett. 75 (1995) 1260 [gr-qc/9504004] [INSPIRE].

[33] C. Cao, S.M. Carroll and S. Michalakis, Space from Hilbert Space: Recovering Geometry from Bulk Entanglement, Phys. Rev. D 95 (2017) 024031 [arXiv:1606.08444] [INSPIRE].

[34] S.-S. Lee, State dependent spread of entanglement in relatively local Hamiltonians, JHEP 05 (2019) 215 [arXiv: 1811.07241] [INSPIRE].

[35] A. Chodos, Dynamical lattice theory, Phys. Rev. D 18 (1978) 3833 [InSPIRE].

[36] T. Konopka, F. Markopoulou and S. Severini, Quantum Graphity: A Model of emergent locality, Phys. Rev. D 77 (2008) 104029 [arXiv:0801.0861] [InSPIRE].

[37] T. Konopka, F. Markopoulou and L. Smolin, Quantum Graphity, hep-th/0611197 [INSPIRE].

[38] T. Banks and W. Fischler, The holographic spacetime model of cosmology, Int. J. Mod. Phys. D 27 (2018) 1846005 [arXiv: 1806.01749] [INSPIRE].

[39] S.-S. Lee, Horizon as critical phenomenon, JHEP 09 (2016) 044 [arXiv:1603.08509] [INSPIRE].

[40] R.L. Arnowitt, S. Deser and C.W. Misner, Dynamical Structure and Definition of Energy in General Relativity, Phys. Rev. 116 (1959) 1322 [InSPIRE].

[41] C. Teitelboim, How commutators of constraints reflect the space-time structure, Annals Phys. 79 (1973) 542 [INSPIRE].

[42] M. Henneaux, A. Kleinschmidt and G. Lucena Gómez, A dynamical inconsistency of Horava gravity, Phys. Rev. D 81 (2010) 064002 [arXiv:0912.0399] [INSPIRE].

[43] H. Weyl, Gravitation and electricity, Sitzungsber. Preuss. Akad. Wiss. Berlin (Math. Phys.) 1918 (1918) 465.

[44] G. Amelino-Camelia, L. Freidel, J. Kowalski-Glikman and L. Smolin, The principle of relative locality, Phys. Rev. D 84 (2011) 084010 [arXiv:1101.0931] [INSPIRE].

[45] P.A.M. Dirac, Generalized Hamiltonian dynamics, Can. J. Math. 2 (1950) 129 [INSPIRE].

[46] C.J. Isham, Canonical quantum gravity and the problem of time, gr-qc/9210011 [INSPIRE].

[47] K.V. Kuchar, Time and interpretations of quantum gravity, in 4th Canadian Conference on General Relativity and Relativistic Astrophysics, G. Kunstatter, D.E. Vincent and J.G. Williams eds., Winnipeg Canada (1992), pg. 211.

[48] J.B. Hartle and S.W. Hawking, Wave Function of the Universe, Phys. Rev. D 28 (1983) 2960 [INSPIRE].

[49] S.-S. Lee, Quantum renormalization group and holography, JHEP 01 (2014) 076 [arXiv: 1305.3908] [INSPIRE].

[50] E. Witten, Multitrace operators, boundary conditions and AdS/CFT correspondence, hep-th/0112258 [INSPIRE].

[51] P. Lunts, S. Bhattacharjee, J. Miller, E. Schnetter, Y.B. Kim and S.-S. Lee, Ab initio holography, JHEP 08 (2015) 107 [arXiv: 1503.06474] [INSPIRE]. 
[52] A. Strominger, The dS/CFT correspondence, JHEP 10 (2001) 034 [hep-th/0106113] [INSPIRE].

[53] S.R. Das and A. Jevicki, Large N collective fields and holography, Phys. Rev. D 68 (2003) 044011 [hep-th/0304093] [inSPIRE].

[54] R. de Mello Koch, A. Jevicki, K. Jin and J.P. Rodrigues, $A d S_{4} / C F T_{3}$ Construction from Collective Fields, Phys. Rev. D 83 (2011) 025006 [arXiv: 1008.0633] [InSPIRE].

[55] D. Marolf, Emergent Gravity Requires Kinematic Nonlocality, Phys. Rev. Lett. 114 (2015) 031104 [arXiv: 1409.2509] [INSPIRE].

[56] C. Hull, Timelike T-duality, de Sitter space, large $N$ gauge theories and topological field theory, JHEP 07 (1998) 021 [hep-th/9806146] [INSPIRE].

[57] R. Bousso, Holography in general space-times, JHEP 06 (1999) 028 [hep-th/9906022] [INSPIRE].

[58] J.M. Isidro, Integrability, Seiberg-Witten models and Picard-Fuchs equations, JHEP 01 (2001) 043 [hep-th/0011253] [INSPIRE].

[59] S. Nojiri and S.D. Odintsov, Conformal anomaly from dS/CFT correspondence, Phys. Lett. B 519 (2001) 145 [hep-th/0106191] [INSPIRE].

[60] E. Abdalla, B. Wang, A. Lima-Santos and W.G. Qiu, Support of dS/CFT correspondence from perturbations of three-dimensional space-time, Phys. Lett. B 538 (2002) 435 [hep-th/0204030] [INSPIRE].

[61] D. Anninos, T. Hartman and A. Strominger, Higher Spin Realization of the dS/CFT Correspondence, Class. Quant. Grav. 34 (2017) 015009 [arXiv:1108.5735] [INSPIRE].

[62] J. Polchinski, Renormalization and Effective Lagrangians, Nucl. Phys. B 231 (1984) 269 [INSPIRE].

[63] C. Rovelli, Partial observables, Phys. Rev. D 65 (2002) 124013 [gr-qc/0110035] [INSPIRE].

[64] B. Dittrich, Partial and complete observables for Hamiltonian constrained systems, Gen. Rel. Grav. 39 (2007) 1891 [gr-qc/0411013] [INSPIRE].

[65] I.R. Klebanov and A.M. Polyakov, AdS dual of the critical $O(N)$ vector model, Phys. Lett. B 550 (2002) 213 [hep-th/0210114] [INSPIRE].

[66] M.R. Douglas, L. Mazzucato and S.S. Razamat, Holographic dual of free field theory, Phys. Rev. D 83 (2011) 071701 [arXiv: 1011.4926] [INSPIRE].

[67] L.A. Pando Zayas and C. Peng, Toward a Higher-Spin Dual of Interacting Field Theories, JHEP 10 (2013) 023 [arXiv: 1303.6641] [INSPIRE].

[68] R.G. Leigh, O. Parrikar and A.B. Weiss, Holographic geometry of the renormalization group and higher spin symmetries, Phys. Rev. D 89 (2014) 106012 [arXiv:1402.1430] [INSPIRE].

[69] R.G. Leigh, O. Parrikar and A.B. Weiss, Exact renormalization group and higher-spin holography, Phys. Rev. D 91 (2015) 026002 [arXiv:1407.4574] [INSPIRE].

[70] E. Mintun and J. Polchinski, Higher Spin Holography, RG and the Light Cone, arXiv: 1411.3151 [INSPIRE].

[71] M.A. Vasiliev, Higher spin gauge theories in four-dimensions, three-dimensions and two-dimensions, Int. J. Mod. Phys. D 5 (1996) 763 [hep-th/9611024] [InSPIRE]. 
[72] M.A. Vasiliev, Higher spin gauge theories: Star product and AdS space, hep-th/9910096 [INSPIRE].

[73] S. Giombi and X. Yin, Higher Spin Gauge Theory and Holography: The Three-Point Functions, JHEP 09 (2010) 115 [arXiv:0912.3462] [INSPIRE].

[74] M.A. Vasiliev, Nonlinear equations for symmetric massless higher spin fields in (A)dS(d), Phys. Lett. B 567 (2003) 139 [hep-th/0304049] [INSPIRE].

[75] J. Maldacena and A. Zhiboedov, Constraining Conformal Field Theories with A Higher Spin Symmetry, J. Phys. A 46 (2013) 214011 [arXiv:1112.1016] [InSPIRE].

[76] J. Maldacena and A. Zhiboedov, Constraining conformal field theories with a slightly broken higher spin symmetry, Class. Quant. Grav. 30 (2013) 104003 [arXiv: 1204.3882] [InSPIRE].

[77] I. Sachs, Higher spin versus renormalization group equations, Phys. Rev. D 90 (2014) 085003 [arXiv: 1306.6654] [INSPIRE].

[78] J. Ambjørn, B. Durhuus and T. Jónsson, Three-dimensional simplicial quantum gravity and generalized matrix models, Mod. Phys. Lett. A 06 (1991) 1133.

[79] N. Sasakura, Tensor model for gravity and orientability of manifold, Mod. Phys. Lett. A 06 (1991) 2613.

[80] M. Gross, Tensor models and simplicial quantum gravity in $>2-D$, Nucl. Phys. Proc. Suppl. 25 (1992) 144.

[81] R. Gurau, Colored Group Field Theory, Commun. Math. Phys. 304 (2011) 69 [arXiv:0907.2582] [INSPIRE].

[82] X.-L. Qi, Exact holographic mapping and emergent space-time geometry, arXiv:1309.6282 [INSPIRE].

[83] J. Maldacena, Non-gaussian features of primordial fluctuations in single field inflationary models, JHEP 05 (2003) 013 [astro-ph/0210603] [INSPIRE]. 